The Astrophysical Journal Supplement Series No. 259, 28:157-209

(c) 1974. The American Astronomical Society. All rights reserved. Printed in U.S.A.

\title{
THE NATURE OF FAINT BLUE STARS IN THE HALO. II.*
}

\author{
Jesse L. Greenstein and Anneila I. Sargent \\ Hale Observatories, California Institute of Technology, Carnegie Institution of Washington \\ Received 1973 December 3
}

\begin{abstract}
Spectra and colors of 189 hot (FB) stars selected colorimetrically and mostly within $30^{\circ}$ of the galactic poles are analyzed quantitatively for surface gravity and effective temperatures. Palomar spectra give line intensities and Balmer line profiles, found in the Appendix tables. Using a network of model atmospheres, the $g$ and $\theta$ so determined give directly the light-to-mass ratio, and eventually the luminosities. The high-latitude hot stars cover a range of $10^{6}$ in luminosity, and are classifiable into various groups.

Spectroscopically normal B stars make up 26 percent of the sample; they rotate and have nearly normal line spectra. Interpreted as Population I stars, on the mass-luminosity relation, they have relatively high luminosities and large $z$-coordinates. For some, the nuclear lifetimes at present luminosities are less than transit times from the plane. Their high velocities present a problem for galactic kinematics. A few are slightly helium rich, while others are highly evolved Population II stars, from details of the spectroscopy. Some of the Population I stars may be in not-understood, shortlived evolutionary phases of high luminosity and low mass.

The $(g, \theta)$-relation can be transformed into luminosity-temperature diagrams if masses are known. Many fall along a relation $\log g \theta^{4}=2.35$, have common properties of halo stars, and $T_{\text {eff }}$ from $9500^{\circ}$ to $40,000^{\circ} \mathrm{K}$. The light-to-mass ratio for these is 68 (in solar units); most $\mathrm{HB}$ and sdB stars have weak metal and weak helium lines, i.e., are halo stars. We call this the extended horizontal branch (EHB). Quantitative classification gives 17 percent of the IFB stars as horizontal-branch $B$ and early $A(\mathrm{HB})$ stars, and 16 percent subdwarf $B$ (sdB) stars. There are 16 percent subdwarf $O$ (sdO) stars, including planetary nuclei; these have strong, or very strong, helium lines. Assuming a constant mass, $0.66 \mathrm{~m}_{\odot}$, the observed $L / \mathrm{m}$ gives $M_{b}=+0.5$. The EHB for field stars fits the globular cluster $\mathrm{HB}$, and extends it to high $T_{\text {eff }}$. The bolometric correction produces the change of $M_{V}$ with color. The hot white dwarfs are 11 percent of the FB stars and appear as a bridge between the sdO stars and the ordinary DA white dwarfs. Composite stars are unresolved, noninteracting binaries with strange $U B V$ colors; they require a faint $M_{V}$ for the primary (e.g., sdO or sdB), and an unevolved $\mathrm{G}$ companion.

The helium deficiency and the evolutionary problems are sharpest for the EHB stars. In a few, slightly stronger helium lines are accompanied by lines of peculiar elements (S III, P II) seen at high dispersion. While the $L / \mathrm{m}$ ratio is closely the same for $\mathrm{sdO}$ as for $\mathrm{sdB}$ and $\mathrm{HB}$, the He lines become strong. Evolutionary tracks avoid the region of the hot EHB stars; the hottest sdO stars approach the helium main sequence. The low surface helium is almost certainly not cosmological in origin. Gravitational diffusion in a nonrotating star in the absence of convection is plausible; in the sdO and the peculiar sdB stars convection and helium flashes may have occurred.

Numerous radial velocities were measured. Where possible, proper motions, luminosities, and radial-velocity dispersions were used for space motions. The Population I normal stars have abnormally high space motions, not greatly different from Population II stars. The luminosities derived from spectroscopy are consistent with those from peculiar motions.
\end{abstract}

Subject headings: faint blue stars - high-velocity stars - horizontal-branch stars - subdwarfs - stellar evolution

\section{INTRODUCTION}

Many hot stars have been found in the galactic polar caps, some representing advanced stages of stellar evolution. After the first discovery lists by Humason and Zwicky (1947), additional surveys for faint blue stars were made using various colorimetric systems. While $U B V$ photometry quickly showed that these stars were unusual and included many white dwarfs, detailed spectroscopic study was necessary to establish the population type, luminosity, temperature, and composition anomalies of these stars. Humason classified low-dispersion spectra of some, and Berger (1963) and Feige (1958) published early classifications of Greenstein's spectra. Extensive qualitative classification was carried out by Greenstein (1960, 1966). The growth of predictions from reliable stellar-

* In part, presented as the Henry Norris Russell Lecture of the American Astronomical Society, delivered at Boulder, Colorado, 1970 June. atmosphere models for hot stars permitted quantitative analyses by Sargent and Searle (1968), Newell (1969), Norris (1970) and others. Since Greenstein had been accumulating a large number of Palomar spectra of faint blue stars since 1952, data on 189 stars were available. Most have photoelectric colors; only a few have reliable motions; many of the hotter stars were observed with the multichannel spectrophotometer to check the temperature calibration.

The method used here is a quantitative spectroscopic and colorimetric one, dependent mostly on $U B V$ colors and hydrogen-line profiles. The Balmer discontinuity is rather insensitive to surface gravity $g$ for $T>10,000^{\circ} \mathrm{K}$, and to the $\mathrm{He} / \mathrm{H}$ ratio. Thus, $Q$, the reddening-free combination of $B-V$ and $U-B$, gives $\theta$, the reciprocal effective temperature, and is used for both reddened and unreddened stars. It is most sensitive to $T$ through the $T$-dependence of the Balmer jump. The $\mathrm{H} \gamma$ and $\mathrm{H} \delta$ profiles predicted from 
hydrogen-line blanketed, LTE (and for hot stars, non-LTE) models are used to determine $g$. To obtain the luminosity we must know the mass, $\mathrm{m}$, which is likely to be in the range $0.4-0.7 \mathrm{~m}_{\odot}$ for old stars in late stages of evolution; we initially adopt a constant mass, thus assuming all to be Population II objects. "Normal" stars found are next analyzed by adopting the normal mass-luminosity relation. Since many of the spectra are of 18 and $38 \AA \mathrm{mm}^{-1}$ dispersion, measures of $\mathrm{He}$, $\mathrm{Mg}$ II, C II, and $\mathrm{Ca}$ II lines were made and assist population discrimination. Apparent composition anomalies, such as low $\mathrm{He} / \mathrm{H}$ and metal/ $\mathrm{H}$ ratios, are expected in Population II stars, and are found. Many apparently normal stars were found, possibly members of the old disk or runaway Population I, mostly among the apparently brighter objects (see § VIII).

A major result is that the luminosity-temperature diagram displays an extended horizontal branch (EHB) stretching to higher temperatures than do globularcluster horizontal branches. These stars have been classified as horizontal-branch stars (HBA and HBB, discussed in $\S \mathrm{IX}$ ) and as subdwarfs ( $\mathrm{sdB}$ and sdO, discussed in $\S \S \mathrm{IX}$ and $\mathrm{X}$ ). But most of these, apparently, belong to one major sequence, with a simple relation between temperature and surface gravity. $\mathrm{He} \mathrm{I}$ and $\mathrm{He}$ II lines are found weak in most halo stars observed, suggesting either low primordial, cosmological $\mathrm{He} / \mathrm{H}$ abundance, or stratification by atomic weight during this evolutionary stage. Among the very hot stars many have strong helium lines, and are helium rich. Section XII on stars with composite spectra shows that unresolved binaries are common, and permits estimates of $M_{V}$ for sdB and sdO stars.

Since a very large body of data is presented, most tabular matter is confined to the appendices, where tables A1 to A6 are lists by separate type. Individual stars are discussed in footnotes to these tables, except for a few important or peculiar objects; table A7 gives new radial velocities. We describe first in $\S$ II the selection process and the observations and measurements; in § III, the determination of atmospheric parameters $(T, g)$ and classification; in $\S \mathrm{IV}$, the determination of luminosities; in $\S \mathrm{V}$, error analysis; in $\S$ VI, systematic effects and the temperature scale for white dwarfs; and in $\S$ VII, the HertzsprungRussell diagram. Later sections describe individual types of stars, while $\$ \S$ XIII and XIV give a brief theoretical discussion.

\section{SELECTION FOR OBSERVATION AND MEASUREMENT}

Selection was almost completely on the basis of blue color, rather than by proper motion. Most stars are within $30^{\circ}$ of the galactic poles; a few high-velocity and otherwise interesting stars were observed nearer to the galactic plane. All were planned to have intrinsic $B-V<0$, but a few proved cooler and difficult to study by our method. The low ratio of $O$ and $B$ stars to $A$ stars made it inefficient to observe stars spectroscopically near $B-V=0.00$ when searching for highly evolved objects in the halo. The colorimetric surveys and the spectra taken are therefore preferentially those of the bluest stars. The bluest stars in globular-cluster horizontal branches seldom reach the high temperatures found in many of the galactic-pole field stars. The $U B V$ colors of hot main-sequence stars, subdwarfs, and white dwarfs all approach those of a blackbody. Distinction between different kinds of hot objects and determination of their helium content near the end of stellar evolution was one major goal. Statistical completeness and uniformity of selection with respect to color were therefore not thought worthwhile. These would have been quite difficult to attain, as frequency distributions of $B-V$ are biased differently in different colorimetric surveys. The true frequency of stars as a function of temperature cannot be derived but should differ from that in our tables only by a smoothly varying weighting factor, favoring hot stars.

Lists from which stars were selected for observation include: Iriarte and Chavira (1957); Chavira (1958, 1959); Iriarte (1959) (the Tonantzintla northern and southern galactic polar surveys), denoted TON and TON-S; Feige (1958), denoted F; Slettebak, Bahner; and Stock (1961), SBS; Haro and Luyten (1962), PHL; Cowley (1958); and Klemola (1962), mostly BD and Astrographic Catalog objects, some with proper motions. Photometric data were largely taken from Eggen $(1968 a, b)$ and the catalog by Blanco et al. (1968); also available were new measures by Margaret Penston (1973), and by Charles Kowal (1973). Recently temperatures were derived from the multichannel spectrometer, especially for the hotter stars. Some proper-motion stars (GD) from the Lowell lists (Giclas, Burnham, and Thomas 1965, 1967), mostly new, hot white dwarfs and hot subdwarfs, were observed because of their very blue color. A few planetary nebulae of low-surface brightness (Abell 1966) had very blue central stars discovered by color and were therefore included. White dwarfs are found in the list by Humason and Zwicky (1947), which was selected only by color. The HZ list includes a zone near the Hyades, at somewhat low latitude, slightly reddened and rich in white dwarfs.

Spectrograms of faint stars from Greenstein's previous studies were available, obtained with the primefocus spectrograph of the 200 -inch $(5-\mathrm{m})$ Hale reflector at dispersions of 190 and $90 \AA \mathrm{mm}^{-1}$. At dispersions of 38 and $18 \AA \mathrm{mm}^{-1}$, spectra were taken at the coudé focus for a number of the brighter stars. Effectively: the magnitude limits, at these dispersions were 16,14 13 , and 11 , respectively. A few of the brightest B stars $(m \leq 10)$ were taken at scales up to $4 \AA \mathrm{mm}^{-1}$.

The stars observed are listed by type in tables $\mathrm{A} 1$ through A6. FB numbers were assigned in order o: right ascension (epoch 1950). The justifications for the classification given are found in later sections. The properties of each class of star could be better em. phasized by dividing the tables according to type Although this makes it necessary to search six tables for a given star, to reduce the length and the size 0 : the tables we do not publish a résumé. The positions finding charts, photometry source, proper motions, anc space motions are available in a card catalog of the 
FB stars, kept in Pasadena. Such information will be provided on request.

Microphotometer tracings on an intensity scale gave mean $\mathrm{H} \gamma$ and $\mathrm{H} \delta$ profiles for each star. The absorption depression at the line center, $R_{c}$, and $D(x)$, its total width in angstroms at an absorption of $x$ from the continuum $(x=0.1$ or 0.2$)$ were tabulated. The principal quantum number, $n$, of the last visible Balmer line was also noted. Because many plates were of sufficiently high dispersion, the strongest lines of $\mathrm{He}$ II

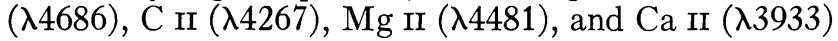
were measured. In $\mathrm{He} \mathrm{I}$, we measured the strongest singlet $(\lambda 4388)$ and triplet $(\lambda 4471)$ lines. These results are given in tables A1-A6. Very approximate upper limits are given (based on the dispersion used) for lines not seen on the tracings. As an indication of the weight to be attached to each set of measurements, the number of plates measured and their dispersions are shown. A dispersion of $18 \AA \mathrm{mm}^{-1}$ is denoted by Pd; of $38 \AA \mathrm{mm}^{-1}$ by $\mathrm{Pe}$; of $90 \AA \mathrm{mm}^{-1}$ by $\mathrm{N} 1$, and of $190 \AA$ $\mathrm{mm}^{-1}$ by N2; Pc is $4.5 \AA \mathrm{mm}^{-1}$. The number of plates used appears after these symbols. Interstellar $\mathrm{Ca}$ II exists in many blue stars in the polar caps, exceeding $0.25 \AA$ equivalent width (Greenstein 1968) and blended with stellar $\mathrm{Ca}$ II in our tabulation.

\section{DETERMINATION OF THE ATMOSPHERIC PARAMETERS}

There are two major parameters to be determined. The first, the temperature, is determined from colors. Photoelectric $U B V$ colors determine the parameter $Q$ (Johnson and Morgan 1953)

$$
Q=(U-B)-0.72(B-V),
$$

which is reddening free and closely correlated with $\theta_{\text {eff }}$, the reciprocal effective temperature. This should not be used for white dwarfs because the Balmer lines depress the $B$ magnitude. A method involving $U-V$, that is, the Balmer jump, was developed. For all other stars we use Hyland's (1967) relation between $Q$ and $\theta_{\text {eff }}$, shown in figure 1 , originally applicable to stars with $-0.87<Q<0.00$. Morton and Adams (1968)

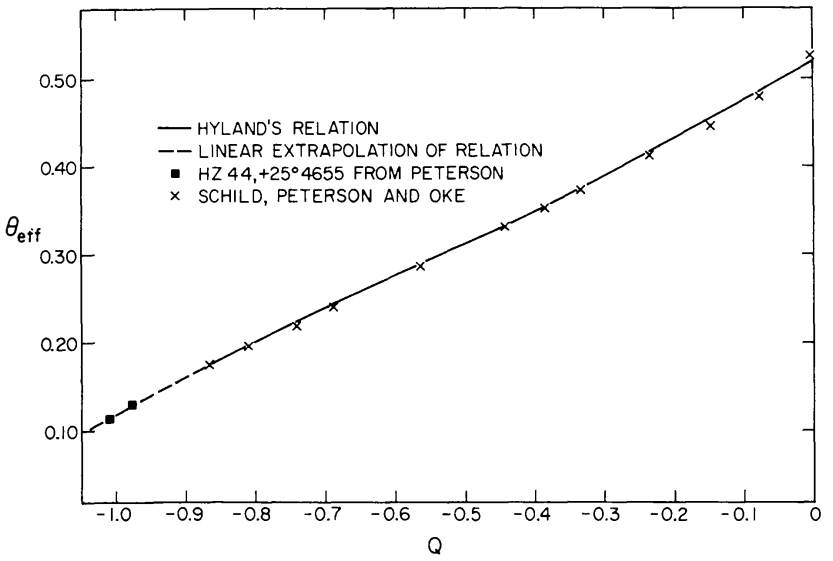

Fig. 1.-The adopted relation between $Q$ and $\theta_{\text {eff }}$ for $\mathrm{B}$ and $\mathrm{O}$ stars, based on Hyland (1967) and with extensions from Peterson (1969). The relation of Schild et al. (1971) is shown by crosses. gave a temperature calibration close to that of Hyland, as does Heintze (1969). Both are for normal (highmetal-content) stars. For $Q>0.00$, the $\theta-Q$ relation is gravity sensitive, since the Balmer discontinuity is reduced by the $\mathrm{H}^{-}$contribution to the opacity and our method does not work. In these cases we made use of Rodgers's (1971) analyses of such cooler stars of both populations. To calibrate hotter stars with $Q<-0.87$ we used $\theta_{\text {eff }}$ determined for HZ 44 and $+25^{\circ} 4655$ by Peterson (1969). These lie near a linear extrapolation of Hyland's relation. Our final $\theta-Q$ relation shows deviations of the order of \pm 0.01 in $\theta$ from that adopted by Norris (1970, p. 101); it coincides almost exactly with the Morton and Adams scale (i.e., is slightly hotter than Hyland at $Q<-0.80$ ). The new fundamental calibration of absolute stellar fluxes by Oke and Schild (1970) was compared with ATLAS models by Schild, Peterson, and Oke (SPO) (1971). Their final adopted effective temperatures, as a function of $Q$, are plotted on figure 1 (crosses). Note the extraordinarily close agreement; unfortunately the very hot end is not yet so calibrated, nor are metal-deficient halo stars.

Preliminary attempts to use the Strömgren uvby indices indicate that a reddening-free calibration with model atmospheres, based mostly on $[u-b]$ may be useful. A study of Graham's (1970) colors of the Feige stars shows that the range of $[u-b]$ is from 1.40 at $Q=0.00$ to -0.15 at $Q=-1.05$. The deviations, dependent on the type of star, are \pm 0.07 in $[u-b]$ from the mean relation. The $\left([u-\bar{b}], T_{\text {eff }}\right)$-diagram for non-LTE model atmospheres by Mihalas (1972a) shows that even $[u-b]$ becomes insensitive ${ }^{1}$ to temperature at $32,000^{\circ} \mathrm{K}$. The $(b-y, Q)$-diagram for the Feige stars shows enormous deviations from the mean relation for white dwarfs, probably because spectral lines affect the narrower bands covered by the Strömgren filters. The multichannel data are preferable, where available. Our temperatures seem to be systematically reliable as high as $T_{\text {eff }}=40,000^{\circ} \mathrm{K}$ (beyond the usefulness of LTE models) but the effect of errors increases rapidly at $T_{\text {eff }}>30,000^{\circ} \mathrm{K}$. The slope of the $\theta-Q$ relation is such that an error of $\pm 0.028 \mathrm{mag}$ in $Q$ gives an error of \pm 0.010 in $\theta$. Since $\bar{U}-B$ is the color most subject to accidental errors and difference between individual observers, we estimate that the best broad-band photoelectric observation gives errors of \pm 0.010 ; and the average, \pm 0.020 in $\theta$. Although the accidental errors may be small, systematic errors do appear between different observers, especially for very hot Tonantzintla stars, for which abnormally negative $B-V$ have been found. The photoelectric errors have increasingly large effects on the deduced luminosity at high temperature. Obviously, this study would have greatly benefited from farultraviolet photometry from space. The systematic errors of interpretation based on model atmospheres depend on the opacity in hot stars and its sensitivity to the $\mathrm{He} / \mathrm{H}$ and $\mathrm{M} / \mathrm{H}$ ratios, which may be population

1 A preprint from Olson shows that revised sensitivity functions increase the usefulness of $[u-b]$ at high $T$, and confirms that differences between non-LTE and LTE continua are small. 
dependent. We will discuss later the reliability of the LTE computations and of the metallic opacity (ultraviolet blanketing correction).

The surface gravity, $\log g$, is derived from the Balmer line widths, $D(x)$, in the line wings. We measured $D(0.1)$ and $D(0.2)$ for $\mathrm{H} \gamma$ and $\mathrm{H} \delta$, and first used theoretical LTE line profiles by Mihalas (1965) for unblanketed models. Based on these we followed the procedure of Sargent and Searle (1968), which we refer to as SS. Since the Stark broadening coefficient, $l(\Delta \lambda)$ varies as $(\Delta \lambda)^{-5 / 2}$, the quantity $D(x)$ varies as $g^{0.2}$. The relationship

$$
\log D(x)=0.2 \log g+C(\theta),
$$

is valid for sufficiently high $g$ and small $x$. Straight lines were fitted at different $\theta$ to the few available models, at $x=0.1$ and $x=0.2$, providing quite reliable extrapolation on the high $g$ side. $C(\theta)$ was chosen to give the best values near $\log g=4$. These approximate relations helped separate the different types of stars in a first solution for $g$ and $\theta$.

Initially in sharp-lined stars $D(0.1)$ was used, since any error of measurement had less effect on $\log g$ than a comparable measure of $D(0.2)$, a smaller quantity. Effects of rotation are also minimized. In stars with sufficiently broad (and usually strong) lines, $D(0.2)$ was preferred since it is less sensitive to errors in locating the stellar continuum. When $D(x)<10 \AA$, or when $\theta<0.17$, errors of measurement and theory will have a large effect, and the $g$ is indicated as doubtful in the tables. In the later reanalysis, $D(0.2)$ was used, and where only $D(0.1)$ was measurable, an approximation $\log D(0.2)=\log D(0.1)-0.28$ was used for hot stars. The effect of finite spectroscopic resolution on the hydrogen-line profiles is to broaden the core; at $90 \AA$ $\mathrm{mm}^{-1}$, the core of the instrumental function is about $2 \AA$ in total width. The quantities $D(x)$ should be reduced by an increasing fraction as they approach $2 \AA$, making $g$ unreliable at low $g$, i.e., in stars with small Stark broadening. At coudé dispersions this effect is negligible. At high $T$, the fractional errors, $\Delta \theta / \theta$, increase, and both $T$ and $g$ become so uncertain that sometimes only upper or lower limits to $T$ could be derived. The $g$ and luminosity of many hot subdwarfs (sdO) are indicated as doubtful in the tables. In addition, helium-rich stars which still show Balmer lines give spuriously low gravities.

The results of the investigation, at an early stage, were presented as the Henry Norris Russell Lecture at the American Astronomical Society, at Boulder, Colorado, in 1970, and in the Annual Report of the Director of the Hale Observatories for 1969-70. However, the rapid increase in the number and variety of model atmospheres available made it advisable to study the effect of improvements in theory. We collected all published, and many unpublished, models and line profiles. We are grateful to many scientists who supplied models and Balmer line profiles long in advance of publication. Shipman (1970, 1972) computed high-g models at Harvard and Caltech with the ATLAS program developed at Harvard and Smithsonian
(Kurucz 1970). Besides those in his thesis he provided unpublished models at strategic high temperatures and high gravity. Since atmospheric helium was known to be low, the $\mathrm{He} / \mathrm{H}$ ratio 0.01 was adopted, both to eliminate the effect of the Pickering series and to prevent excessively low ultraviolet opacity. (This does not affect the predicted flux or Balmer lines but accelerates convergence.) The same program was run by us, both with and without Balmer and Lyman line blanketing. Shipman's (1970) results for high $g$ calibrate equation (2) and help define the turn down at the left side of the $(D, g)$-plane. In figure 2 we show the calibration curves from older models, with wide gaps bridged by plausible interpolation. The thesis by Norris (1970, pp. 93, 125-127), used a scaled $T(\tau)$ relation by Mihalas, Morton, and co-workers (see Norris 1970, pp. 20-22, for details and references). Deriving $\log g$ at a given $\theta$ from $D(0.2)$, near $\log g=4$, theoretical profiles by Norris give $\log g$ about 0.35 larger than the first set we used. Results by Searle and Rodgers (1966), pioneers in this type of analysis, for the cooler B and A stars in NGC 6397 agree moderately well.

By early 1972, extensive new models were available and a complete restudy became possible. This was largely carried out when one of us (J.L.G.) was on leave as Professor at NORDITA, in Copenhagen. Klinglesmith (1971) published hydrogen-line blanketed models, based on his own program and ESW broadening theory. Compared with Mihalas (1965) his equivalent widths are lower at a given $T$; we would therefore have obtained higher $g$, for a given $T$ and $D(0.2)$. Such difficulties must occur in comparison of unblanketed and blanketed models. Klinglesmith's wide-band colors seem wrong, presumably based on poor instrumental response curves. His model fluxes, however, are close to the ATLAS results. He kindly sent us printouts of Balmer line profiles, based on his models, as well as some results for $\log g=5.25$ and 6.00 , from $\theta=0.50$ to 0.125 , from unpublished models.

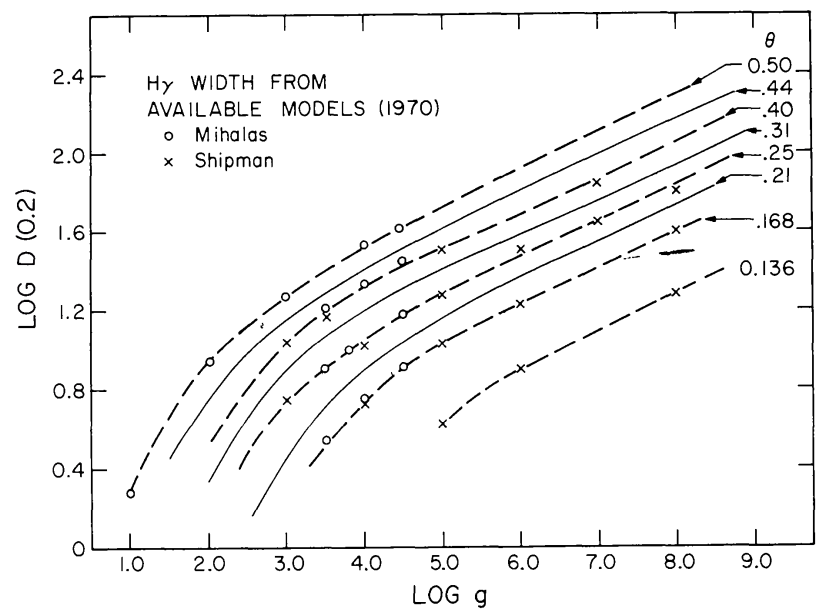

FIG. 2.-The models used in 1970 for the Russell Lecture, from Mihalas (1965) and Shipman (1970) with the $D(0.2)=g^{1 / 5}$ approximation. 
Deane Peterson has computed a large useful grid of hydrogen-line blanketed LTE models at Stony Brook, using the ATLAS program. He gave us quite complete data on fluxes and Balmer profiles from $\theta=0.59$ to $\theta=0.18$, mostly for $\log g=3.5$ to 4.5 , for $\mathrm{He} / \mathrm{H}=$ 0.05 and 0.10 . He omits the Pickering lines of He II. $\mathrm{He}$ was also kind enough to compute profiles at log $g=5,6,7,8$ for $\theta=0.36-0.18$, especially for this investigation. We are grateful to him for his data and continued advice. Finally, a set of important non-LTE (and LTE) models were constructed by Mihalas $(1972 a, b)$ for $\theta=0.33-\theta=0.092$, mostly for $\log g=$ $3-4.5$. These are not blanketed, use the Vidal, Cooper, and Smith (1973) broadening theory, and compute the non-LTE populations of the first five levels of hydrogen; while using $\mathrm{He} / \mathrm{H}=0.10$, he also omits the Pickering lines. The helium content makes little difference to our conclusions. The final $D(0.2)$ based on all the above models, as used, are plotted in figure 3. From this large body of model atmospheres, reliable theoretical bolometric corrections and continuum fluxes are derived which agree remarkably well. The line-broadening theories differ, but apparently do not produce substantial effects. The non-LTE effects on line profiles, however, are serious at low $\log g$ and $\theta \leq 0.167$; the wings of the lines tend to go into emission in LTE, but they remain in absorption, and the line deepens and strengthens in non-LTE. Fortunately, we have few stars of low $\log g$. For $\theta \leq 0.20$ there is no significant difference in $D(0.1), D(0.2)$, or fluxes, between LTE and non-LTE, from the Mihalas tables. However, for the hot subdwarfs with weak hydrogen absorption, the Mihalas non-LTE results give quite different surface gravities. At $\theta=0.125, \log g=4.5$, in non-LTE the central depth $R_{c}=0.36$, and $D(0.2)=4.4 \AA$; in LTE, $R_{c}=0.20$ and $D(0.2)=0.0$. Mihalas $(1972 b)$ finds $T_{\text {eff }}$ to increase by $3000^{\circ} \mathrm{K}$ at type 09 . The overlap between Peterson and Mihalas is only in the $15,000^{\circ}-$

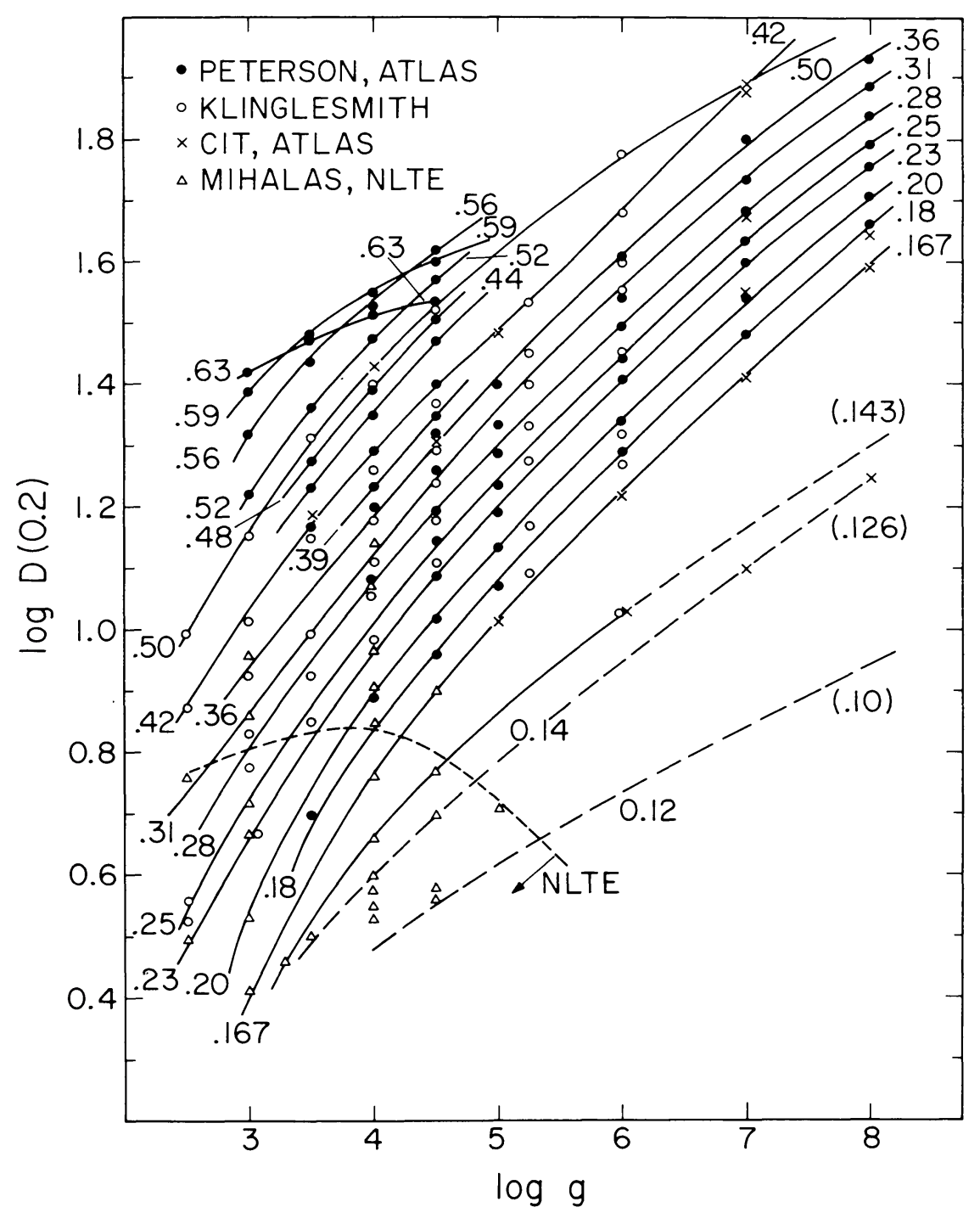

FIG. 3.-The relations used between $D(0.2)$ and $\log g$ for various $\theta$. Models are from Mihalas (non-LTE), and LTE models are by Klinglesmith, Oinas, Peterson, and Shipman (references in text). Most are with the ATLAS program. The dashed locus at low $D(0.2)$ is a suggested limit of applicability of LTE models. 
$28,000^{\circ} \mathrm{K}$ temperature range, where non-LTE effects are small; the differences are thus small at temperatures typical of middle B stars. Peterson also supplied an estimate of the surface gravity at which non-LTE effects become important, based on average collisional/ radiative excitation rates. This is also plotted in figure 3 ; Balmer lines with $\log D(0.2)<0.85$ lie in the nonLTE region; these have $\log g<4$ at $\theta>0.17$. Apparently non-LTE will still be important for $\log g \simeq 5$ at $\theta<0.13$. Unfortunately, no non-LTE models exist at high $T$ and $g$. Figure 3 is only an arbitrary, rough sketch for the hottest stars.

We have compared the temperature, $\theta_{\text {eff }}$, at which $\mathrm{H} \gamma$ has the same width, $D(0.2)$, in Peterson (ATLAS, $X=0.71)$, Klinglesmith $(X=0.67)$, and Mihalas (1972a) ( $X=0.71$, non-LTE) by studying the deviations from Peterson. The systematic differences are, in $\theta_{\mathrm{K} l}-\theta_{\mathrm{P}}$, at $\log g=4,+0.015$ in the range $0.48>\theta>$ 0.20 . The differences $\theta_{\mathrm{M}}-\theta_{\mathrm{P}}$ average +0.015 for $0.30>\theta>0.20$. This agreement is somewhat illusory, since the Mihalas non-LTE models are unblanketed, and have a slightly different color and Balmer jump than a blanketed model at the same $\theta_{\text {eff }}$. The differences $\theta_{\mathrm{K} l}-\theta_{\mathrm{P}}$ at $\log g=5$ are +0.004 .

We face a difficult problem in the relation between the true effective temperature of a star and the effective temperature that labels the model atmosphere. For Population I stars this has been considered by SPO in connection with the absolute calibration of the scale of stellar fluxes (Oke and Schild 1970). The model atmospheres (ATLAS) are line blanketed and include $\mathrm{C}$ I and $\mathrm{N}$ I continua; real stars have additional metalline blocking and metal continuum opacities. They find a quite systematic run of differences $\Delta \theta=\theta_{\text {adopted }}-$ $\theta_{\text {ATLAs. }}$. The "adopted" temperatures were adjusted to fit the far ultraviolet fluxes of Population I stars by Morton and Adams (1968), where metallic line blocking must be included. Blanketing, in a hot star, makes the color temperature (Paschen slope) higher; a blanketed ATLAS model with the same color and Balmer jump as a real star is labeled by a hotter effective $T$. SPO shows that $\Delta \theta$ is near zero at $\theta>0.42$, and may be again zero at $\theta<0.16$, where most visible and nearultraviolet blanketing effects are negligible. In our analysis of the data we first use $T_{\text {adapted }}=T_{\text {eff }}$ from $Q$, and compute $\log g$ from $T_{\text {eff }}$, using the curves of figure 3 . In a second solution we have also computed $\log g^{\prime}$, by reading figure 3 , at a given $D(0.2)$, with $T_{Q}=T_{\text {ATLAS }}+\Delta T\left(T_{\text {eff }}\right)$. Over the range $0.18 \leq \theta_{\text {eff }} \leq$ 0.42 , we have an increase in $T_{\text {AtLAS }}$, which means that for a given line strength, $\log g^{\prime}>\log g$. The $\Delta T$ arises, presumably, from the blocking of ultraviolet flux by metallic lines. It could be zero for Population II stars if they had both low metallic and oxygen-magnesium abundances, and also zero again near A0 (where the energy maximum is in a relatively line-free region). We assume that $\Delta T=0$ at $\theta<0.18$ because of the high level of metallic ionization, and because no calibration, from space, of the ultraviolet radiation of these very hot stars yet exists. The run of $\Delta \theta$ was taken as shown in table 1. The SPO "adopted" scale fits the directly
TABLE 1

Differences in Temperature Labeling of Model Atmospheres Caused by Blanketing

\begin{tabular}{crrr}
\hline \hline$\theta_{Q}$ & \multicolumn{1}{c}{$\Delta \theta$} & \multicolumn{1}{c}{$\theta_{Q}$} & \multicolumn{1}{c}{$\Delta \theta$} \\
\hline$\leq 0.17 \ldots \ldots$ & 0.000 & $0.23-0.27 \ldots \ldots$ & -0.040 \\
$0.18 \ldots \ldots$ & -0.010 & $0.28-0.32 \ldots \ldots$ & -0.030 \\
$0.19 \ldots \ldots$ & -0.015 & $0.33-0.36 \ldots \ldots$ & -0.020 \\
$0.20 \ldots \ldots$ & -0.025 & $0.37-0.40 \ldots \ldots$ & -0.010 \\
$0.21 \ldots \ldots$ & -0.028 & $\geq 0.41 \ldots \ldots \ldots$ & 0.000 \\
$0.22 \ldots \ldots$ & -0.034 & & \\
\hline
\end{tabular}

measured angular diameters of the Population I stars measured with the Narabri interferometer (Hanbury Brown et al. 1967).

The very hot subdwarfs and the white dwarfs present other special problems. There are no non-LTE, high- $g$ models and even no LTE models for high $g$ at $T>$ $28,000^{\circ} \mathrm{K}$. Yet many of the stars are so blue, according to $U B V$ photometry that $\theta$ apparently approaches the value for infinite temperature. At moderate $g$, Mihalas (1972b) finds a gravity dependence of temperature in the uvby photometry. Hot white dwarfs are given excessively high temperatures from $Q$, and probably also in Shipman's (1972) use of $U-V$. In $\S \mathrm{VI} a$ we discuss how we have calibrated temperatures of white dwarfs.

Once $\theta$ and $g$ are known, using the nonlinear relations of figures 1 and 3 , the results shown in figures 4 and 5 can be displayed. The stars are now classifiable quantitatively into various subgroups, ranging from white dwarfs to normal stars. Visual, qualitative classifications had been improvised and used by Greenstein for many halo stars included in this investigation. A working preliminary description divides most of the stars into groups, with data given separately for each group in the Appendix tables:

1. Essentially normal, main-sequence (MS) B or A, (table A1). A2).

3. Globular cluster, horizontal-branch HBB, (table A2).

4. Subdwarfs, sdB (table A3) and sdO (table A4).

5. White dwarfs, (table A5).

6. Composite spectra, (table A6).

7. Rarer types, unclassifiable, or with strong abundance anomalies, or emission lines.

Some stars of group 1 are apparently metal and helium weak, as is $\lambda$ Boo at lower temperature. Visual recognition of HBA (group 2) at 90 or $190 \AA \mathrm{mm}^{-1}$ depends on the visibility of many sharp, deep hydrogen lines, to $n=16$, and sometimes weak $\lambda 3933$, Ca II or $\lambda 4481, \mathrm{Mg}$ II. HBB stars showed $n=12-14$, sometimes weak $\mathrm{He} \mathrm{I}$ lines, and shallower $\mathrm{H}$ lines. Older classifications of the brighter Feige stars by Greenstein, and by Sargent and Searle (1968), recognized sdB stars by abnormally broad $\mathrm{H}$-lines for their blue color, seen only to $n=12$, and possibly weak He I. 
lines; the SS class $\mathrm{Bw}$ has sharper $\mathrm{H}$-lines than their weak $\mathrm{He}$ sdB stars to $n=15$ or 16 , and weak He I lines; these are mostly our HBB stars. Their, and our, sdO stars have broader $\mathrm{H}$-lines seen to $n=10-12$, than do MS stars of the same very blue color, and show $\lambda 4686$ of He II. Some sdO stars have essentially no hydrogen, so both visual and quantitative methods fail. We call stars with $5 \leq \log g \leq 6$ subdwarfs. White dwarfs (Greenstein 1969) of type DA show very broad $\mathrm{H}$ lines to $n=8-10$; the blue DAwk stars often found in colorimetric surveys have colors indistinguishable from sdO stars, main-sequence O stars, or blackbodies, but show shallow $\mathrm{H}$ lines stopping at $n=7-8$. We will call stars with $\log g>7$ white dwarfs.

The composite stars (group 6) are discussed here for the first time. Their $U B V$ colors often lie near the blackbody line and spectra in the blue show only hydrogen, a shallow K-line, and a G-band. They may be confused spectroscopically with HBB but the colors differ since horizontal-branch stars fall on the $(U-B$, $B-V$ )-locus for MS stars. Composites with faint secondaries may look like sdB or sdO stars, and the color differentiation is more difficult. Our methods for determining $T$ and $g$ are totally inapplicable to com-

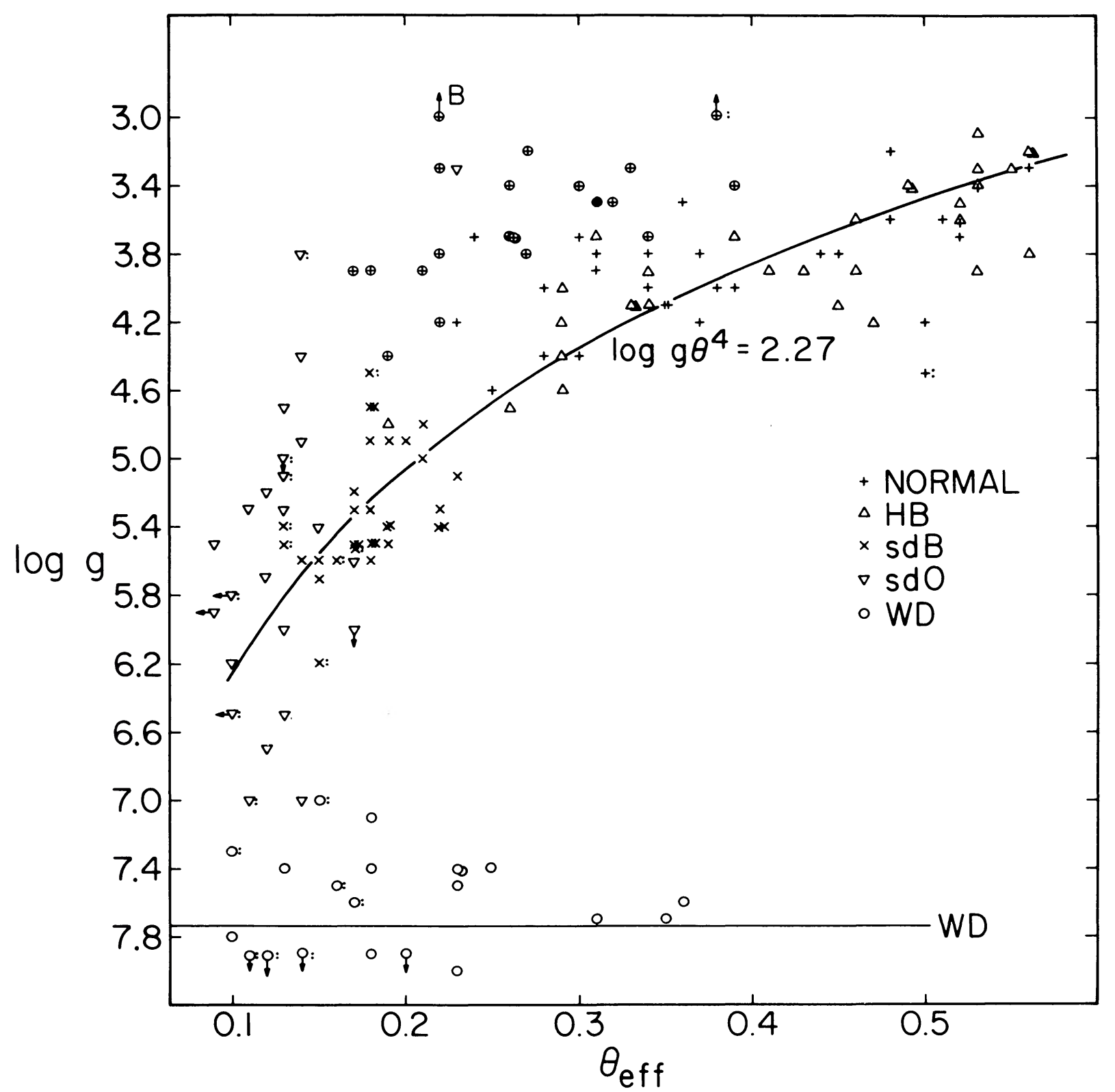

Fig. 4.-The observed relation between $\log g$ and $\theta$. The symbols, used throughout, are: + , normal; $\Delta$, horizontal branch; $\times$, subdwarf $\mathrm{B} ; \nabla$, subdwarf $\mathrm{O}$; and $\mathrm{O}$, white dwarf. " $\mathrm{B}$ " indicates the position Barnard 29 would occupy. The relation $\log g \theta^{4}=2.27$ is shown, as is the hot end of the white dwarfs from Eggen and Greenstein. The arrows indicate direction of likely errors; colons, uncertain measures; and circles around normal stars indicate $\log g$ as possibly uncertain because of high helium or low mass. 


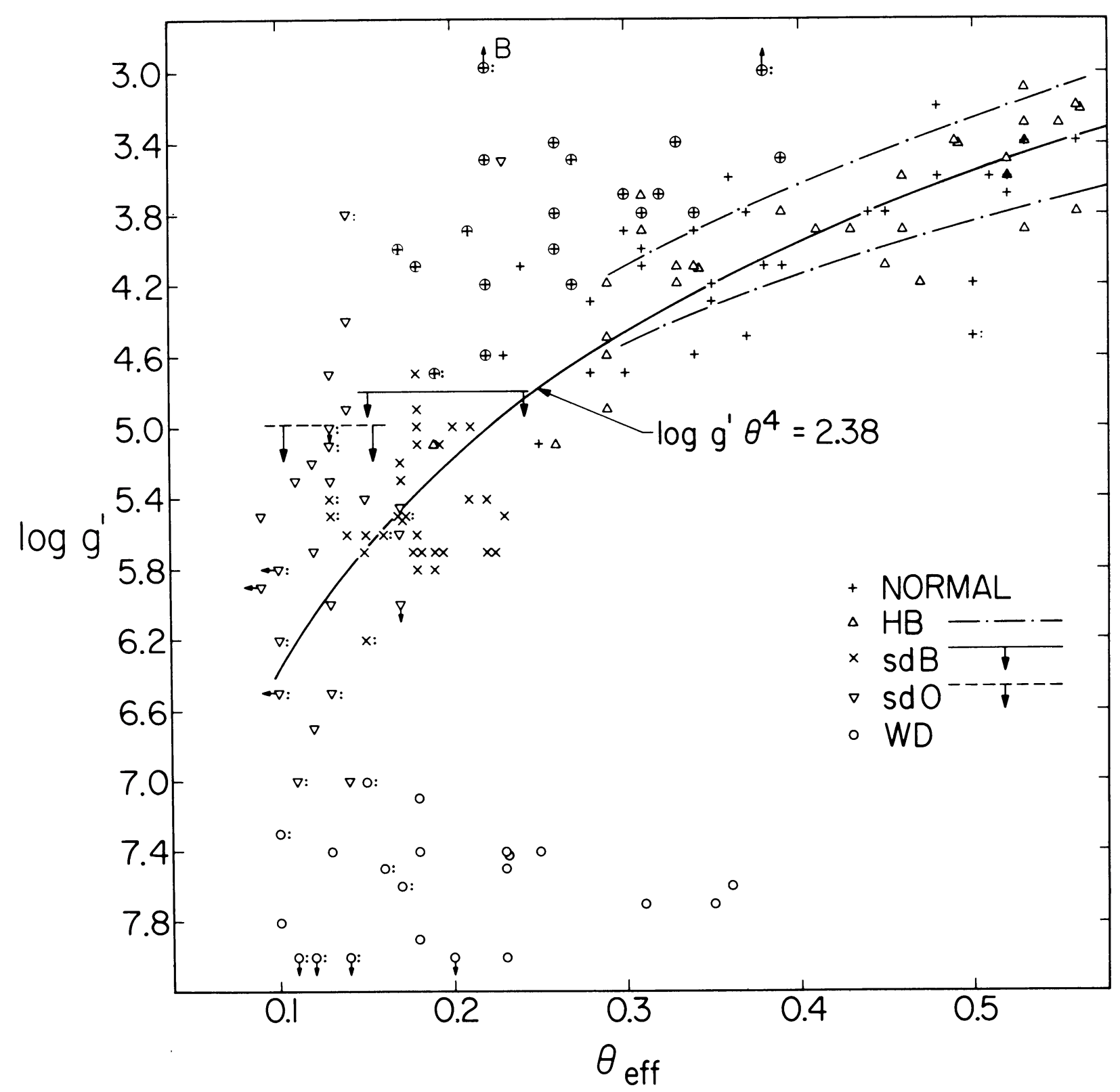

FIG. 5. - The observed relation between $\log g^{\prime}$ and $\theta$, where corrections to the ATLAS temperature are made to allow for ultraviolet line blanketing. The heavy, solid line shows $\log g^{\prime} \theta^{4}=2.38$. The dashed and solid bars with downward pointing arrows indicate our limits of regions for sdO and sdB stars. Most of our HB stars lie between the dashed-dotted lines.

posite stars. In group 7 should be included stars like Barnard 29 in M13, which lie between the horizontal branch and the main sequence. We wish to separate clearly hot stars with gravity less than the main sequence, from the horizontal branch (HBB) and subdwarfs. We therefore include them among the normal stars of table A1, discussed in \$ VIII. Many are abnormal Population II stars, and are discussed in detail in that section.

\section{THE DETERMINATION OF LUMINOSITIES}

The use of $Q$ and $D(x)$ gives $T_{\text {eff }}$ and $\log g$; the distribution of these parameters is shown in figure 4 .
From this, a quite natural grouping of stars of apparently diverse spectra is derivable by use of quantitative methods. These give first the $L / \mathrm{m}$ ratio, and by assuming a mass, give $L$ and the bolometric absolute magnitude, $M_{b}$; from $T_{\text {eff }}$ and the models, $\Delta M_{b}$ is known, so that the $M_{V}$ can be obtained. We might have contented ourselves with obtaining an $L / \mathfrak{m}$ ratio, but we find that many stars are grouped so as to suggest that they have the same mass. We therefore will try to determine actual luminosities. For some stars, the space motion vectors $U, V, W$ help define the population type and luminosity. The bolometric luminosity is obtained from 


$$
M_{b}=M_{b \odot}+2.5 \log \frac{g \mathfrak{m}_{\odot}}{g_{\odot} \mathfrak{m}}\left(\frac{\theta}{\theta_{\odot}}\right)^{4} .
$$

Substituting $M_{b \odot}=4.62 \theta_{\odot}=0.86$ and $\log g_{\odot}=4.44$,

$M_{b}=2.5 \log g+10 \log \theta-2.5 \log \frac{\mathfrak{m}}{\mathfrak{m}_{\odot}}-5.82$.

The absolute visual magnitude is

$$
\begin{array}{r}
M_{V}=2.5 \log g+10 \log \theta-2.5 \log \frac{\mathfrak{m}}{\mathfrak{m}_{\odot}}-5.82 \\
-\Delta M_{b},
\end{array}
$$

where $\Delta M_{b}$ is the bolometric correction. Using these expressions, the spectroscopic absolute bolometric (eq. [4]) and visual (eq. [5]) magnitudes of all stars were calculated assuming $\mathrm{m}=0.66 \mathrm{~m}_{\odot}$. These $M_{V}$ are given in tables A2 through A5 in the Appendix. We will describe later the derivation of the $M_{V}$ of table A1.

Newell (1969) observed 90 stars and assigned them to various categories according to their position on the $\log g$ versus $\theta_{\text {eff }}$ diagram for globular-cluster and halo field stars. In particular, he suggested the existence of two types of horizontal-branch stars, i.e., halo and disk-population horizontal branch. Unfortunately, our sample of stars is too hot for us to make any remarks concerning this division. Most of his stars were relatively bright, with fewer hot horizontal-branch stars, fewer sdO stars, and one white dwarf. We have used our own criteria in classifying our stars rather than Newell's regions. The resulting approximations to specifically defined regions are shown in figure 5 , our diagram of $\log g^{\prime}$ versus $\theta_{\text {eff }}$. The general division of halo stars into normal, (MS), horizontal branch, and subdwarfs corresponds both to what we find quantitatively, and to Greenstein's (1966) older, visual classifications. The main sequence and horizontal branch cross at late $B$, creating an ambiguous region (Newell's group F). Further, not all of our "normal" stars are spectroscopically normal, but show weak $\mathrm{He}, \mathrm{Mg}$ II, i.e., are like Sargent and Searle's Bw group, or $\lambda$ Boo.

Our data in figures 4 and 5 show that a large fraction of the stars are well grouped along a curve which crosses the main sequence. This curve is such that the luminosity is constant, if all the stars have the same mass. From equation (3) or (4), the locus of constant $M_{b}$ is a quartic:

$$
\begin{aligned}
\log g+4 \log \theta & =\log \frac{m}{m_{\odot}}+2.33 \\
& +0.4 M_{b}=\text { constant } .
\end{aligned}
$$

This quartic is sketched in figures 4 and 5 so as to fit best the body of data for HBA, HBB, and sdB stars, without and with the $\Delta T$ correction between ATLAS and SPO. In our earlier work we had used $\log g \theta^{4}=$ 2.35; from equation (6) this corresponds to $M_{b}=$ +0.50 for $m=0.66 m_{\odot}$. Parallel quartics can be drawn corresponding to other mass or luminosity values. To determine the best value is somewhat difficult, in that the values of $\log g$ become quite sensitive to errors, at high temperature, and a straight- foward least-squares fit may not be desirable since the vector change in $\log g$ for an error in $\theta$ lies nearly along the horizontal branch. Taking $\theta$ as the more important, and therefore independent, variable, we group the $\log g$, form $\left\langle\log g\left(\left\langle\theta^{4}\right\rangle\right)\right\rangle$ and derive the constants as shown in table 2. Note first that the masses are appreciably less than the Sun, with luminosities not far from those of RR Lyrae variables, or globular-cluster horizontal branches. The $\log g^{\prime}$ scale gives higher masses at a given luminosity, or, for a fixed mass, about 0.2 fainter $M_{b}$. The range of uncertainty is increased if the sdO stars are included, but the conclusions are not radically changed, i.e., many of the sdO stars have nearly the same luminosity as the HBA, HBB, and sdB stars. Masses of evolved stars of the halo population should be less than $1.0 \mathrm{~m}_{\odot}$ and not less than $0.4 \mathrm{~m}_{\odot}$ (a low-mass white dwarf), and all of the groupings, and assumed luminosities, in table 2 satisfy these conditions. We adopt a constant $\left\langle\log g \theta^{4}\right\rangle=+2.35$ for most of this paper, i.e., $0.66 \mathrm{~m}_{\odot}$ and $M_{b}=+0.5$, and call this locus the extended horizontal branch (EHB). Only 14 of the $63 \mathrm{HBA}, \mathrm{HBB}$, and sdB stars deviate by more than 0.4 in $\log g$ from this relation (agreement is less close for the sdO stars). From equations (4) and (5), this corresponds to $< \pm 1.0 \mathrm{mag}$. This provides from field stars a possible zone of constant luminosity from $9500^{\circ}$ to $40,000^{\circ} \mathrm{K}$ effective temperature, a leftward extension of the globular-cluster horizontal branches. The various subtypes of stars classified visually and quantitatively as $\mathrm{HBA}, \mathrm{HBB}$, and $\mathrm{sdB}$ then would become one temperature sequence. Along this locus an increase of $g$ with increasing temperature justifies the Sargent and Searle (1968) classification of $\mathrm{HBB}$ and sdB stars.

The globular-cluster horizontal branches vary in extent toward the blue. They may have different metal compositions, ages, and possibly different HB masses, which depend on details of red-giant evolution. In figure 6 we show the $M_{V}$ deduced from our EHB locus, as compared to the clusters. The horizontal-branch loci in M3, M13, M15, and M92 were determined from fitting their turnoff points. Using the photoelectric photometry of Sandage (1969), and involving

\begin{tabular}{|c|c|c|c|c|c|}
\hline Group & Datum & $N$ & $\left\langle\log g \theta^{4}\right\rangle$ & $M_{b}$ & $\mathfrak{m} / \mathfrak{m}_{\odot}$ \\
\hline $\begin{array}{l}\text { HBA, HBB, } \\
\text { sdB } \ldots \ldots\end{array}$ & $\log g$ & 63 & 2.27 & $\left\{\begin{array}{l}0.7 \\
0.5 \\
0.3 \\
0.7\end{array}\right.$ & $\begin{array}{l}0.46 \\
0.55 \\
0.66 \\
0.59\end{array}$ \\
\hline Same. & $\log g^{\prime}$ & 63 & 2.38 & $\left\{\begin{array}{l}0.5 \\
0.3\end{array}\right.$ & $\begin{array}{l}0.71 \\
0.85\end{array}$ \\
\hline $\begin{array}{l}\mathrm{HBA}, \mathrm{HBB} \\
\mathrm{sdB}, \mathrm{sdO} \ldots\end{array}$ & $\log g$ & 83 & 2.19 & $\left\{\begin{array}{l}0.7 \\
0.5 \\
0.3 \\
0.7\end{array}\right.$ & $\begin{array}{l}0.38 \\
0.46 \\
0.55 \\
0.51\end{array}$ \\
\hline Same. & $\log g^{\prime}$ & 83 & 2.32 & $\left\{\begin{array}{l}0.5 \\
0.3\end{array}\right.$ & $\begin{array}{l}0.62 \\
0.74\end{array}$ \\
\hline
\end{tabular}
the complex problems of distance moduli based on

TABLE 2

Fits of Quartic to the Extended HORIZONTAL BRANCH 


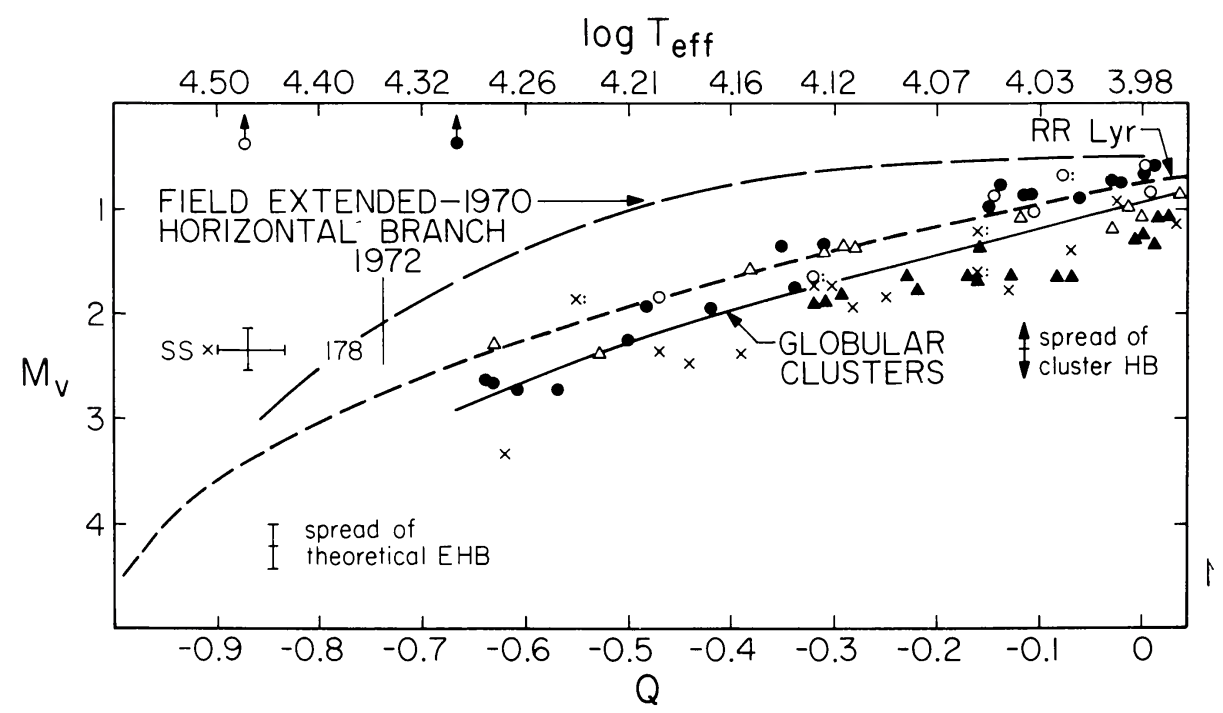

Fig. 6.-The globular-cluster blue-horizontal-branch stars are shown from Sandage's photoelectric measures with his moduli; M13, 14.70 , is represented by filled circles; M3, 15.28, by open circles; M15, 14.88 by crosses; M92, 14.18, by filled triangles. Open triangles represent Cannon and Stobie's results for NGC 6752, with a less certain modulus, 13.4. The theoretical fit for constant $M_{b}=+0.5$ and $\left\langle\log g \theta^{4}\right\rangle=2.35$ is shown for the extended horizontal branch. Spreads in the cluster branches and the plausible range of the constants and masses are given. The open and filled circles, with arrows, at the top of the diagram, are the bright stars at $M_{V}=-0.3$ in $\mathbf{M} 3$ and $M_{V}$ $=-1.5$ in M13. FB 178 is a bright southern sdB. SS $X$ is the temperature of M13 SA-19, a sdB star measured by Sargent and Searle. The adjacent error bars are those of Sandage.

faint-star photometry, it is very satisfying to see how well these clusters define the same region of the $\left(M_{V}, Q\right)$ plane by their horizontal branches. Cannon and Stobie (1973) recently gave photoelectric magnitudes and an approximate modulus of 13.5 for NGC 6752 . If we correct the modulus to 13.4 , then 10 more blue stars can be plotted in figure 6 , useful since, although the absolute modulus is not calibrated, the cluster is rich in blue stars. Their hottest star has $Q=-0.63$ and lies slightly above the other cluster stars, indicating a somewhat flatter $\left(Q, M_{V}\right)$-relation than Sandage's clusters.

Because of the improved ATLAS models in the range $10,000^{\circ}-20,000^{\circ} \mathrm{K}$ (where non-LTE effects are negligible), the present larger constant, $\log g \theta^{4}=2.35$ with an adopted mass of $0.66 \mathrm{~m}_{\odot}$, reproduces the average globular cluster data with extraordinary fidelity. The bolometric correction causes the decreasing $M_{V}$ with increasing $T$. Note that for $\log g \theta^{4}=2.35$ the light-to-mass ratio is $\log \left(L / L_{\odot}\right)\left(\mathrm{m} / \mathrm{m}_{\odot}\right)^{-1}=1.83$. The ratio of luminosity to mass is an observed quantity independent of assumptions concerning the mass. Only the choice of stars affects the constant determined by fitting equation (6) to the observed field-star $\theta$ and $g$ values. Compared with the 1970 reduction of the same data, $\log \left(L / L_{\odot}\right)\left(\mathfrak{m} / \mathfrak{m}_{\odot}\right)^{-1}=1.97$, the change of 0.14 is largely due to the model atmospheres. The effect of the correction $T_{\text {eff }}$ to $T_{\text {ATLAS }}$ is one which increases $g$ over a certain range of $\theta$, but both figures 4 and 5 are nearly equally well fitted by the same constant with $\log g^{\prime}>\log g$ for part of the $T$ range. Table 2 shows that the increase in the constant is of the same order as the effect of the new models.
Those stars which seem to be normal, especially the hotter objects, have lower $g$ than the extended horizontal branch. Their luminosity would be quite incorrect if derived with the same low mass used in our first solution. To proceed, assume that our normal stars obey the normal mass-luminosity relation (Harris, Strand, and Worley 1963),

$$
M_{b}=4.6-10 \log \left(\mathrm{m} / \mathrm{m}_{\odot}\right) .
$$

If the stars are on the unevolved main sequence, $T_{\text {eff }}$ is the best guide to the mass. We had evaluated $M_{b}$ from $g, \theta$ by assuming $0.66 \mathrm{~m}_{\odot}$; the first correction to this value, $\delta M_{b}$, is, in fact, obtained by combining equations (7) and (4).

$$
\delta M_{b}=0.25 M_{b} \text { (main sequence) }-1.6 .
$$

The initial $M_{b}$ (main sequence) is obtained from the zero-age main sequence (ZAMS) as a function of temperature. Since equation (4) involved the measured $g$, we are not forcing the star to lie on the main sequence, but by preserving the observed value of $g$, retain the relative surface gravities of the normal stars. With a new value $M_{b}\left(0.66 \mathrm{~m}_{\odot}\right)+\delta M_{b}$, we enter equation (8) again, find $\delta M_{b}^{\prime}$, the final $M_{b}$, and hence $M_{V}$ given for normal stars in table A1. Note that the decision that a star is spectroscopically "normal" depends on our value of $g$. The effect of any error in $g$ becomes magnified in the final $M_{b}$, as does the error in temperature, since it gives the first $\delta M_{b}$ (eq. [7]). However, certain other information may exist which strengthens our confidence in this decision: e.g., rotation and line strengths of $\mathrm{Si}$ II, $\mathrm{Mg}$ II, and $\mathrm{He}$ I. 


\section{ERROR ANALYSIS}

The effect of accidental errors in $Q$ and $D(x)$ on the $\theta, g, M_{b}$ derived can be estimated analytically for a simple case, based on a linear relation (eq. [2]) between $\log D(x)$ and $\log g$. Inspection of figure 2 shows that at high $g, C(\theta)$ can be approximated as (constant - 0.14 $\left.\theta^{-1}\right)$. From equation (2) an error in temperature, $\Delta \theta$, at fixed $D(0.2)$ produces an error in $\log g$ of $-5 \Delta C(\theta)$, which is $-0.7 \Delta \theta / \theta^{2}$. This term proves larger than the direct effect of the temperature on the luminosity of a star. The composition of errors leads to a term of the form

$$
\frac{\Delta \theta}{\theta}\left(\text { constant }-\theta^{-1}\right)
$$

After evaluating the approximate fit to the $C(\theta)$ with the above constants, and converting into bolometric magnitudes, equation (9a) can be differentiated to give the errors as

$$
\begin{aligned}
\partial M_{b}= & 5.4 \Delta D(0.2) / D(0.2) \\
& +1.75 \Delta \theta / \theta\left(2.48-\theta^{-1}\right) .
\end{aligned}
$$

The temperature-sensitive term in equation (9b) vanishing at $\theta=0.40$ is not physically significant; it arises only from the approximation for $C(\theta)$. Equation (9b) shows that, on the linear theory, errors increase steeply as $\theta$ decreases. At $\theta=0.40, \partial M_{b}$ is zero; at $\theta=0.25, \partial M_{b}=-4.4 \Delta \theta / \theta ;$ at $\theta=0.15, \partial M_{b}=-7.3$ $\Delta \theta / \theta$. The slope of the $(\theta, Q)$-relation is 0.4 ; an error in $U-B=+0.05$ mag produces an error, $\Delta \theta=$ +0.02 and $\mathrm{a} \partial M_{b}$ of $-0.4 \mathrm{mag}$ at $\theta=0.25$ and of $-1.0 \mathrm{mag}$ at $\theta=0.15$. In the $\left(M_{b}, \theta\right)$-plane, this corresponds to a vector inclined upward and to the right, not far from the direction of the EHB. The asymmetry of the effect is also appreciable, i.e., the vector downward to the left is larger. The error produced by \pm 0.025 changes in $\theta$ is shown in the fourth column of table $3 \mathrm{~A}$, for four different temperatures, based on the linear expression (9b). Entries $\partial M_{b}$ were also computed near the horizontal branch, by selecting $\left(\theta_{0}, \log g_{0}\right)$ such as to give the tabulated $\log D(0.2)$. The temperature alone was changed, by letting $\theta$ change by \pm 0.025 and \pm 0.05 . The $\log g$ were read as accurately as possible, given the coarse network of available models. The nonlinearity of the $[\log D(0.2)$, $\log g$ ]-relation changes the effects of errors of $\theta$, as is shown in the other columns of the same table, 3A. The results are quite rough, have no zero at $\theta_{0}=0.40$, and are quite asymmetric. The size and asymmetry of the effect of the actual errors is found to change. Temperature errors cause diffusion of points more or less along the EHB. They could shift stars from the far end of the horizontal branch (sdB) into the sdO (entry $\theta=0.15$, table $3 \mathrm{~A}$ ). We will find this a most sensitive area affecting the apparent structure of the $\mathrm{H}-\mathrm{R}$ diagram from $30,000^{\circ}$ to $40,000^{\circ} \mathrm{K}$. Certainly,

TABLE 3

\begin{tabular}{|c|c|c|c|c|c|c|c|}
\hline \multirow[b]{2}{*}{$\begin{array}{c}\log D \\
(0.2)\end{array}$} & \multicolumn{3}{|c|}{$\partial M_{b}$} & \multicolumn{4}{|c|}{$\partial M_{b}$ FROM MODELS, FOR $\Delta \theta$} \\
\hline & $\theta_{0}$ & $\log g_{0}$ & $\begin{array}{c}\text { Eq. } 9 \mathrm{~b} \\
\Delta \theta=+0.025\end{array}$ & -0.05 & -0.025 & +0.025 & +0.05 \\
\hline $\begin{array}{l}1.24 \ldots \\
1.22 \ldots \\
1.16 \ldots \\
1.05 \ldots\end{array}$ & $\begin{array}{l}0.40 \\
0.30 \\
0.20 \\
0.15^{*}\end{array}$ & $\begin{array}{l}3.94 \\
4.44 \\
5.15 \\
5.65\end{array}$ & $\begin{array}{r}0.00 \\
-0.12 \\
-0.55 \\
-1.22\end{array}$ & $\begin{array}{c}+0.22 \\
+0.33 \\
+0.89 \\
\ldots\end{array}$ & $\begin{array}{l}+0.18 \\
+0.19 \\
+0.29 \\
+1.35\end{array}$ & $\begin{array}{l}-0.26 \\
-0.08 \\
-0.15 \\
-0.82\end{array}$ & $\begin{array}{l}-0.64 \\
-0.03 \\
-0.39 \\
-0.98\end{array}$ \\
\hline
\end{tabular}

EFFECTS OF ERRORS

A. $\partial M_{b}$ Near Horizontal Branch

B. Cooler Stars, Constant $D(0.2), \partial M_{b}$ (from models) for $\Delta \theta$

\begin{tabular}{ccccc}
\hline \hline $\log D(0.2)$ & $\theta_{0}=0.36$ & $\theta_{0}=0.42$ & $\theta_{0}=0.48$ & \multicolumn{1}{c}{ Remarks } \\
\hline $1.5 \ldots \ldots \ldots$ & +0.75 & 0.00 & -0.25 & Near $\log g_{0}=5$ \\
$1.3 \ldots \ldots \ldots$ & +0.40 & 0.00 & -0.47 & Near $\log g_{0}=4$ \\
$1.0 \ldots \ldots \ldots$ & +0.21 & 0.00 & -0.12 & Near $\log g_{0}=3 ;$ non-LTE region \\
\hline
\end{tabular}

C. At Fixed $\theta$, Changes in log $g$ Caused by Errors. in $\log D(0.2)$

\begin{tabular}{ccccc}
\hline \hline $\log D_{0}(0.2)$ & $\theta=0.50$ & $\theta=0.36$ & $\theta=0.28$ & $\theta=0.20$ \\
\hline $1.40 \ldots \ldots \ldots$ & 3.89 & 4.96 & 5.53 & 6.33 \\
$1.50 \ldots \ldots \ldots \ldots$ & 4.36 & 5.46 & 6.02 & 6.80 \\
$1.60 \ldots \ldots \ldots$ & 4.92 & 5.95 & 6.54 & 7.32 \\
$0.85 \dagger \ldots \ldots \ldots$ & 2.03 & 2.70 & 3.14 & 3.82 \\
$0.90 \ldots \ldots \ldots$ & 2.18 & 2.86 & 3.32 & 4.00 \\
$1.00 \ldots \ldots \ldots$ & 2.47 & 3.23 & 3.69 & 4.41
\end{tabular}

* This region of fig. 3 is poorly defined because of incompleteness of high- $T$, high- $g$ models.

† Note smaller $D(0.2)$ change; models unavailable. 
any real gaps will be blurred. The apparent abrupt end of the EHB in a vertical sdO sequence is far from correct; the stars may lie far to the left of our diagram, since very blue stars all have the same $Q$. We will return to other criteria of temperature later.

An error of \pm 10 percent in line width, $D(0.2)$, or an equivalent change in line-broadening theory, produces an error of \pm 0.5 mag, vertically. (Details are given in tables 3B and 3C.) Locating the continuum is difficult for shallow lines, but 10 percent errors are the maximum plausible. The correction for finite spectroscopic resolution increases as the lines weaken (low $g$ or high $T$ ); their neglect means that such stars should be intrinsically brighter. Few sdO stars have bright apparent magnitude, and as a group, sdO stars are most subject to this error. At $\log g=5.5, \theta=0.17$, $D(0.2)=10 \AA$; the instrumental profile, at $190 \AA$ $\mathrm{mm}^{-1}$, increases the width to about $14 \AA$, and produces an error $\delta M_{b}=+1.6 \mathrm{mag}$. In Population I objects which may have become "runaway" B stars, at $\theta=$ 0.40 , and $\log g=2.5$, the instrumental correction is about the same as in sdO stars.

Deeper theoretical uncertainties are worst in the curved, lower left-hand portion of the $[D(x), \log g]$ plane. The coefficients in equation (9b) will change. Too few models in non-LTE at high $T_{\text {eff }}$ exist, where systematic errors might be serious. The very hottest models only poorly define the link between the whitedwarf models and the Mihalas non-LTE models. The lower right-hand part of figure 3 is only a rough sketch of reality, and errors may be large.

Finally, a systematic error arises from composition anomalies. Two of the highest-luminosity stars (FB 37 and FB 40) are carbon rich, probably helium rich, and therefore hydrogen poor. They are found to have $M_{b}=-4$ and $-7, \log T_{\text {eff }}=4.36$. For such stars the decrease in $\mathrm{H} / \mathrm{He}$ abundance may be expected to weaken the Balmer lines, producing an illusion of low g. Furthermore, the normal mass-luminosity relation may not apply, i.e., the $\delta M_{b}$ from equation (8) is quite incorrect. For some helium-rich sdO stars, luminosities cannot be given since the $\mathrm{H} \gamma$ profiles are affected by the Pickering series.

\section{SYSTEMATIC EFFECTS}

\section{a) The Temperature Determination for Hot White Dwarfs}

From extensive observational and theoretical work on white dwarfs, temperatures, gravities, and radii are now being reliably determined. Such studies, with spectroscopic and colorimetric surveys, however, revealed that some white dwarfs were indeed very hot and relatively luminous. These naturally were included in our first FB lists; it seemed less valuable to study many already well-known DA stars, which had also been discovered by their proper motion. Those still included are, on the average, quite hot, have relatively weak Balmer lines, and some also have $\mathrm{He}$ I or $\mathrm{He}$ II lines. The border between sdO, DO, and DAwk groups is quite uncertain, but in our list of $27 \mathrm{FB}$ white dwarfs there are 20 with $\log g \geq 7.0$.

We could not use $Q$ as a temperature indicator, since the Balmer lines fill a large portion of the $B$-filter bandpass. For an increase $\Delta B$ in apparent magnitude so produced, $\Delta Q=-1.72 \Delta B$, and easily attains 0.4 or $0.5 \mathrm{mag}$. The temperature determined from the $U-V$ color, however, is sensitive to the Balmer jump, which is, in large part, a function of temperature only (unless $\mathrm{He} / \mathrm{H} \gg 1)$. The equations (2) and $(9 \mathrm{a}-9 \mathrm{~b})$ in our error analysis apply best to the white dwarfs. The major problem then concerns only the temperature. Shipman (1972), using Oke's (1974) multichannel spectrophotometer data, gives a temperature scale, and also calibrates the $U-V$ colors. For some stars he used the ubvy data by Graham (1972). Trimble and Greenstein (TG, 1972) restudied and adjusted Shipman's scale. Wickramasinghe and Strittmatter (WS, 1972) made extensive use of the Graham ubvy colors. Weidemann (1971) had already pointed out the significant reduction in observational scatter in the $(u-b, b-y)$-plane with Graham's colors, as compared to that in the $(U-B, B-V)$-plane. He deduced that $\log g$ must lie in a narrow range; thus $\mathrm{m} / R^{2}$ is nearly constant. From the degenerate gas $(\mathfrak{m}, R)$-relation for constant $\mu_{e}$, therefore, we expect $R$ to be constant. TG noted (1972) that on the Shipman scale there remained a systematic run of $\langle\log R\rangle$ with temperature. If his highest temperatures are reduced, this effect disappears. The revised Shipman temperatures used by TG can also be compared with those of WS. The agreement is essentially complete, with the largest difference in log $T_{\text {eff }}=0.07$, for 11 stars from $4.0<\log T_{\text {eff }}<4.4$.

The 27 white dwarfs left in the FB list now have basically improved temperatures, based on both different model atmospheres (Shipman used ATLAS; WS used their own hydrogen-blanketed models) and different colorimetric techniques. Inspection of table A5 shows that many FB objects are too hot to rely on these calibrations. The $\left\langle T_{\text {eff }}\right\rangle=27,000^{\circ} \mathrm{K},\langle\theta\rangle=0.18$, with a range from 0.10 to 0.36 . We also still lack a fine grid of high-g, hot model atmospheres. However, the results seem to be internally consistent. The $\langle\log g\rangle=$ 7.65 , with a dispersion $\sigma= \pm 0.57$ for 11 stars with $\theta \leq 0.18 ;\langle\log g\rangle=7.72$, with $\sigma= \pm 0.42$, for nine with $0.19 \leq \theta \leq 0.36$. The suggested temperatures should be checked by other methods and new models; when this is done these hot white dwarfs will provide calibrations for other stars at the upper end of the white-dwarf cooling curve. Special studies are needed of the helium opacity, the contribution of He II Pickering series to the Balmer lines, and the temperatures of the hot helium-rich stars, e.g., FB 6, FB 51, FB 70, FB 88, and FB 114.

\section{b) The Systematic Effects in Surface Gravity}

In $\S$ III we discussed uncertainties in models and the difference in $T_{\text {eff }}$ between blanketed and unblanketed stars. Figures 4 and 5 showed both $\log g$ and $\log g^{\prime}$ as a function of their respective temperature scales. Study of the best data for HBA, HBB, and sdB stars gives the least-squares fit (see table 2) for the constant in $\log g^{\prime} \theta^{4}=2.38 \pm 0.06$ and $\log g \theta^{4}=2.27 \pm 0.04$. Individual stars scatter more from the $\log g^{\prime} \theta^{4}$, i.e., 
\pm 0.16 as compared to \pm 0.11 from the $\log g \theta^{4}$ relation. Part of this increase arises from nonsmooth corrections $\Delta T\left(T_{\text {eff }}\right)$ to $T_{\text {ATLAs. }}$. Nevertheless, since the body of the FB stars are halo population, metal-poor stars, it seemed best to adopt as a mean $\log g \theta^{4}=2.35$ for the EHB stars, in determining the $L / \mathfrak{m}$ ratio and ultimately the mass used. However, for the entire body of the stars we use $\log g$, and $T_{\text {ATLAS }}$ directly from $T_{Q}$, so that a single, definite procedure has been followed. We also have available all the results on the $\log g^{\prime}$ scale. We have prepared H-R diagrams using both $\log g$ and $\log g^{\prime}$ and find them substantially the same, except for the slightly lower luminosities derived using $\log g^{\prime}$. The only place where the $\log g^{\prime}$ results are taken into account, therefore, is in the mass determination of the EHB stars, based on fitting the EHB to the globular clusters (fig. 6). However, we use the same mass (0.66 $\mathrm{m}_{\odot}$ ) for all halo stars (except the normal stars). This may be less well-justified for sdO stars, since the higher luminosity objects, like planetary nuclei, may be evolved old-disk stars. Even then, masses greater than $i \mathrm{~m}_{\odot}$ seem unlikely, and produce only $0.45 \mathrm{mag}$ change in $M_{b}$.

Another source of error, and a more serious one, is the effect of assuming high masses, from the normal mass-luminosity law, for the "normal" stars. The most

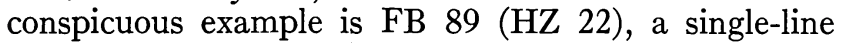
spectroscopic binary (Greenstein 1973) with a most probable mass of $0.5 \mathrm{~m}_{\odot}$. It has $M_{b}=-2.7, M_{V}=$ +0.1 . However, since the star is included in the "normal" group spectroscopically, the high mass arising from the procedure of equations (7) and (8) results in it being quite incorrectly ascribed $M_{b}=-6.1$. Another similar case is HD 137569 (FB 158) studied by Danziger and Jura (1970). Their $\theta, \log g$ are 0.42 , 2.3 ; our method gives $0.38,2.4$; because of the low gravity the star, if called "normal," has $M_{V}=-5.5$, which is clearly incorrect. FB 158 is almost certainly a highly evolved velocity variable, of lower mass and correspondingly lower luminosity, as Danziger and Jura find. But even with $0.66 \mathrm{~m}_{\odot}$ (used for the halo stars) the resultant luminosity is $M_{b}=-3.6, M_{V}=$ -2.6. The star $+33^{\circ} 2642$ (FB .162) has $\theta=0.22$, log $g=2.3$; treated as a normal star $M_{b}=-10.0, M_{V}=$ -7.7; even if it has $0.66 \mathrm{~m}_{\odot}, M_{V}=-3.9$. FB 162 has been studied by Traving (1962) and likened to Barnard 29 in M13 (see Stoeckly and Greenstein 1968). It has low abundance of $\mathrm{C}, \mathrm{N}, \mathrm{O}, \mathrm{Mg}$, and $\mathrm{Si}$, and slightly low He. Traving derives a mass of 0.2 to $0.7 \mathrm{~m}_{\odot}$ for such stars. However, low gravity forces us to include them among "normal" objects. We do not know which fainter objects of this type are still to be found in our "normal" star list. At low resolution, the weakness of C II and $\mathrm{Mg}$ II cannot be firmly established; even such a criterion would not exclude FB 89, which has normal metallic-line strengths. Finally, objects like FB 37, FB 40, carbon-rich stars, for a different reason, are also unreliable. They have high $\mathrm{C} / \mathrm{H}$ ratios and might therefore have high $\mathrm{He} / \mathrm{H}$ ratios, like $\mathrm{R} \mathrm{CrB}$. Both the $(\theta, Q)$ - and the $[\log g, D(0.2)]$-relations would be incorrect.

\section{c) Systematic Effects in the Spectrophotometry}

A clearly central issue is the reliability of the Palomar spectrophotometric calibration. Earlier studies (Wright et al. 1964) had suggested that the combination of the coudé spectrograph and its calibration wedge at Palomar produced, for the few stars there measured, slightly lower equivalent widths $(\sim-15$ percent $)$ than did the Victoria spectrographs. The Mount Wilson 100inch $(2.5-\mathrm{m})$ coudé, with its own calibrations, also had lower equivalent widths $(\sim-6$ percent $)$ than did Victoria. Photoelectric scans at the 100 -inch roughly confirm the photographic results there (Wright et al. 1964 , table 9b). Clearly, there is no guarantee that the Victoria scale is a correct one. The calibration spectrographs at Mount Wilson and Palomar are of the same design, using a triangular slit, grating, and lens system. The Palomar calibration device was used for both coudé and prime-focus spectra; the prime-focus spectrograms (N1 and N2 scales) would suffer from the same calibration error, but would have different scattered light corrections. Because of lower resolution they show smaller central absorptions, $R_{c}$, than the coudé. Our general impression is that no large-scale error exists in the photometry, nor any large difference in scattered light, between the Pd, Pe series and the N1, N2 series. A most profitable external comparison is via Feige stars observed by Sargent and Searle (1968) at $48 \AA$ $\mathrm{mm}^{-1}$ at Lick Observatory; the Lick calibration was not tested by Wright et al. (1964). There are 22 stars in common; for some we had $18 \AA \mathrm{mm}^{-1}$ spectra, for others as low as $190 \AA \mathrm{mm}^{-1}$. The central absorptions on Palomar 18 and $38 \AA \mathrm{mm}^{-1}$ spectra were measured as 90 percent of those measured on the Lick plates For a triangular line profile our expected line widths $D(0.1)$ or $D(0.2)$ would consequently be reduced by 10 percent. In fact, the correlation of the $D(0.2)$ values from Palomar and Lick shows about a 6 percent reduction for Palomar. This is sufficiently close, given the differences in dispersion, to suggest that the maximum error in calibration, spectrograph and personal bias in drawing the continuum is less than 10 percent, and may be as low as 6 percent. If we take 8 percent, the effect of such an error would be to change $\log g$ from its "true" value by about 0.2 , although we do not know which is the "true" value. Correction for such systematic error increases our absolute magnitudes by about $0.5 \mathrm{mag}$. From table 2 this reduces masses on the EHB by 20 percent. Our "normal" stars lie on the average somewhat above the main sequence, indicating that any change in $\log g$ should be in the sense that it is increased. However, low $g$ could also arise from evolutionary effects.

The line profiles and equivalent widths were all measured by one of us (A. I. S.) on Palomar spectra, or quoted from other papers by one of us, with the following exceptions. In table A1, the data for FB 162 including $W, g, \theta$ are from Traving (1962). In table A3, FB 9 is from Baschek, Sargent, and Searle (1972); FB 178 , a far-southern sdB, is taken from Baschek and Norris (1970). In table A4, measurements for the sdO FB 49, 150, 179 are from Peterson (1969), but FB 131 


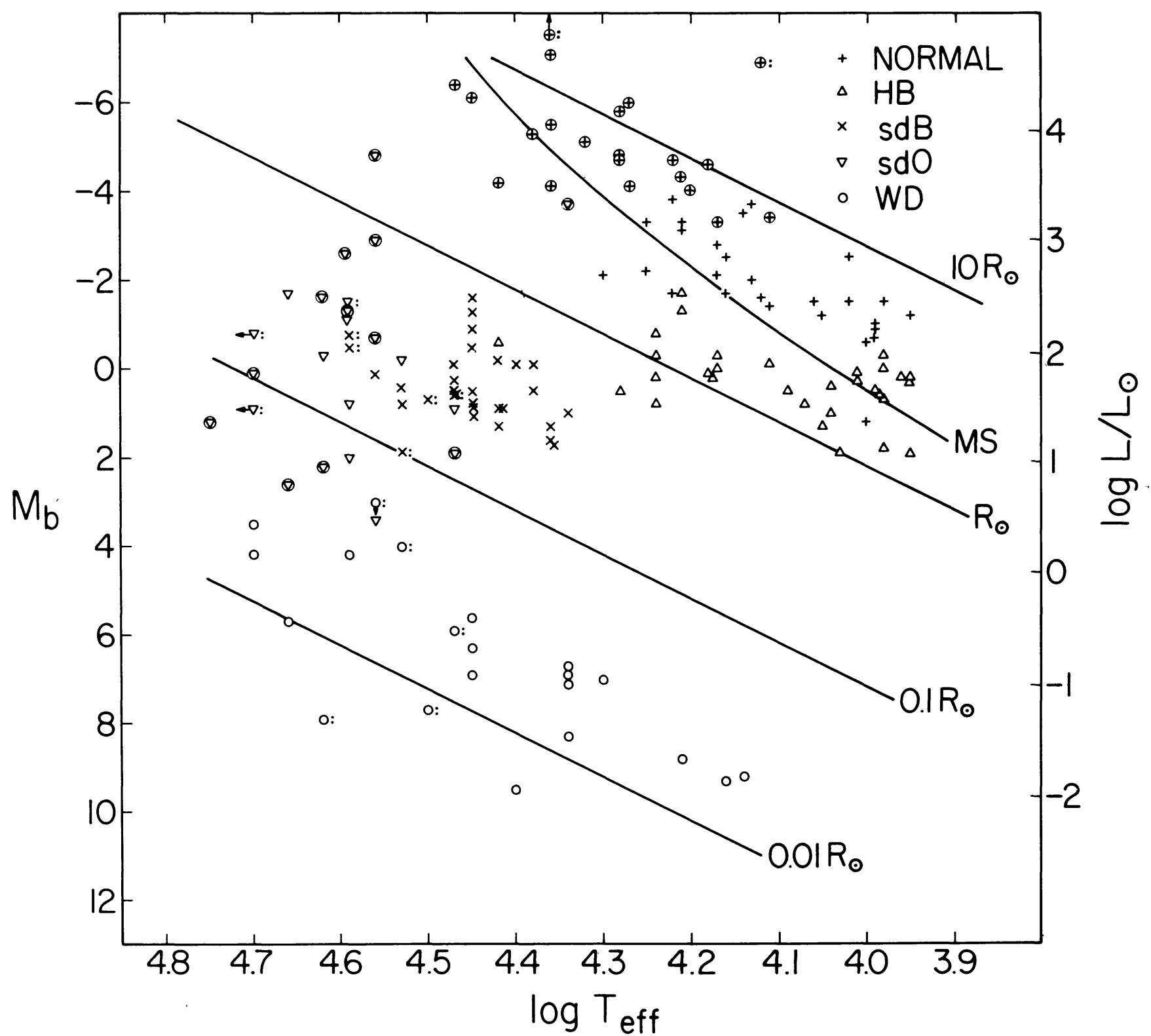

FIG. 7.-The $\left(M_{b}, \log T_{\text {eff }}\right)$-diagram, with $L / L_{\odot}$, radii, and the main sequence (MS). For the EHB stars, $\mathfrak{m} / \mathrm{m}_{\odot}=0.66$ was assumed; for the normal stars, the normal mass-luminosity relation. Circles surrounding normal and sdO stars indicate values suspect because of strong helium lines.

represents new measurements. For all we have recomputed $\theta, g, M_{b}, M_{V}$ by our methods, for consistency. For FB 162 we did not measure the available plates, which were the same Traving used, taken by one of us (J. L. G.).

A number of our spectra were measured about 10 years ago by Mildred Shapley Matthews. Comparison of the measured $D(0.2)$ by A. I. S. and M. S. M. shows that a $45^{\circ}$ line is the best fit to the data. What significant differences exist ( $\sim 15$ percent) are in the sense that $D(0.2)$ by A. I. S. is somewhat greater than $D(0.2)$ by M. S. M. Finally, we have measured an excellent recent Mount Wilson spectrum of FB 187 covering the range of equivalent width 60-6400 mA. The
$\langle\Delta \log W\rangle=+0.028 \pm 0.032$, with the Palomar strengths the larger. The $D(0.1)$ and $D(0.2)$ at Palomar were also measured larger by about 15 percent; the residual intensities of $\mathrm{H} \gamma$ and $\mathrm{H} \delta$ were the same. The comparison is satisfying, given the difference in dispersion, $18 \AA \mathrm{mm}^{-1}$ at Palomar, and $10 \AA \mathrm{mm}^{-1}$ at Mount Wilson. The velocities agreed within $3 \mathrm{~km} \mathrm{~s}^{-1}$. FB 187 is a rotating, more or less normal star.

\section{THE IUMINOSITY-TEMPERATURE DIAGRAMS}

Figures 7 and 8 display the bolometric and visual luminosities of the FB stars. The $M_{V}$ for normal and other stars were derived as described previously. Colons indicate uncertain results either because of the data, or 


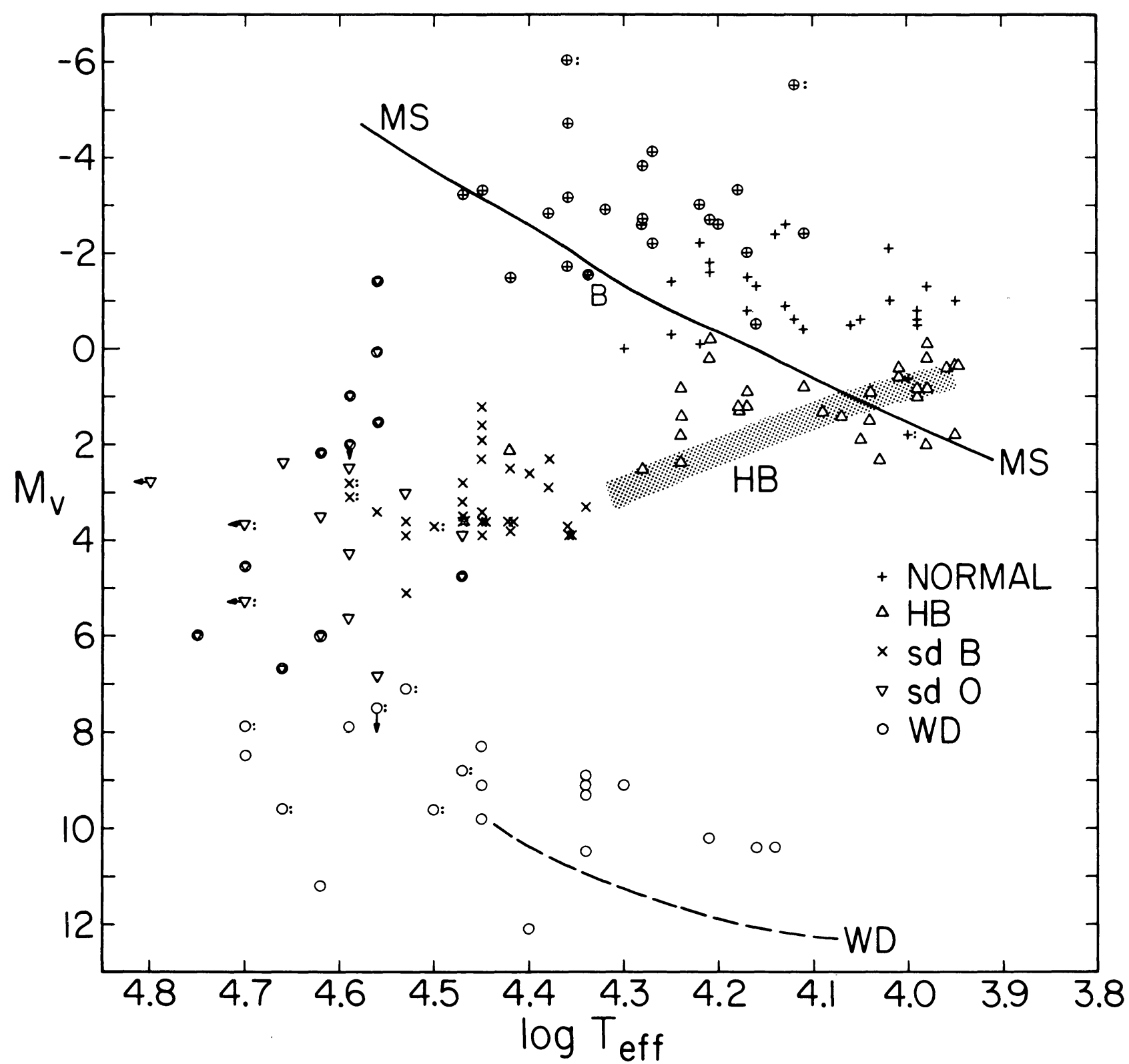

Fig. 8.-The $\left(M_{V}, \log T_{\text {eff }}\right)$-diagram, with the main sequence, white dwarfs, Barnard 29 (B), and the globular-cluster horizontal branches shown.

uncertainty as to the nature of the star. Arrows show the direction, but not the size, of probable corrections. In figure 7 , with $M_{b}$ and $\log T_{\text {eff }}$ as coordinates, the loci of constant radii are shown; the main sequence is slightly curved. The "normal" stars are quite luminous, and have radii between $10 R_{\odot}$ and that of the main sequence, i.e., some are evolved or have been ascribed too large a mass. The extended horizontal branch reaches from $\mathrm{HBA}$ to sdO types with radii from 3 to $0.1 R_{\odot}$. In figure 8, using $M_{V}$, the downward curvature of the EHB is caused by the bolometric correction. In both, for $10,000^{\circ}<T<30,000^{\circ} \mathrm{K}$, a large gap separates the white dwarfs from all halo stars. Normal white dwarfs lie near the $0.01 R_{\odot}$ line, which fits our new data. Some sdO stars lie near the left end of the EHB. An apparently vertical group of sdO stars near $T=40,000^{\circ} \mathrm{K}$ may represent an evolutionary track from various precursor states to the white dwarfs, or be an artifact of our determination of temperatures from colors. All but the "normal" stars have been plotted with an assumed mass of $0.66 \mathrm{~m}_{\odot}$.

The general features are fairly well as expected from stellar-interior theory except perhaps for the EHB. Short-lived evolutionary tracks for double-shell-source low-mass stars $\left(0.6-1.2 \mathrm{~m}_{\odot}\right)$ traverse the top of the H-R diagram horizontally (e.g., Paczyński 1971b). After some thermal pulses and loops they decay in luminosity 
on nearly vertical tracks at $T_{\text {eff }} \simeq 10^{5}$, dropping from $M_{b}=-3$ to +2 . The cooling lifetime of white dwarfs at their highest luminosity is near $10^{7}$ years, the horizontal-branch life $10^{8}$ years, and white dwarfs as faint as $0.01 L_{\odot}$ are at least $5 \times 10^{8}$ years old. The lifetimes of planetary nebulae are estimated near $2 \times$ $10^{4}$ years; the decay times from high luminosity through the so-called ultraviolet-dwarf stage, if shortened by neutrino emission, is less than $4 \times 10^{5}$ years (Stothers 1966; Paczyński 1971b). Therefore, in spite of high luminosity we expect few or no planetary nuclei (either active or extinct), or very hot white dwarfs in our limited sample. Theory must provide the connection between the high-luminosity planetary nuclei $(T>$ $\left.10^{5}{ }^{\circ} \mathrm{K}, M_{b}<-5\right)$ and the white dwarfs, and especially the presence of hot horizontal-branch stars $\left(T>20,000^{\circ}\right.$ $\mathrm{K})$ which are rare in globular clusters, but favored here by selection.

From a stellar-statistical point of view we must now recognize the great diversity in luminosity in the faintblue star population, $15 \mathrm{mag}$ in figure 7 . While the FB stars contain many genuinely normal stars, the largest number of stars are in advanced evolutionary stages. The overall mean visual luminosity is $+2.2 \pm 0.3$ (m.e.), with a range of $\pm 9.8 \mathrm{mag}$. However, the frequency of $M_{V}$ is clearly not a normal Gaussian; the high maximum near $M_{V}=+3$ contains the hot end of the $\mathrm{HBB}$, the $\mathrm{sdB}$, and the $\mathrm{sdO}$ stars. The EHB perturbs the luminosity function over a wide range of $M_{V}$, as does the horizontal branch (late B to G, including RR Lyrae variables) in a globular cluster. Figure 9 shows $N\left(M_{V}\right)$ for FB stars from the various lists in the tables, as well as the means for each group. If the white dwarfs are omitted, then the $\left\langle M_{V}\right\rangle=+1.00 \pm 0.23$, and the dispersion is $\pm 2.6 \mathrm{mag}$. We will describe each type of star separately, and give a critique of our results. Later we will discuss the implications for stellar evolution.

\section{NORMAL STARS}

\section{a) General Properties}

Table A1 contains the data on the normal stars, luminosities, temperatures, $\log g$, line profiles, and equivalent widths. These also define our scale of normal line strengths used later in a discussion of the helium abundances in other types of FB stars. We do not give conventional spectral types in table A1 for normal stars, although they are available. Stars with $M_{V}$ marked with an asterisk are suspected of various peculiarities, and will be discussed later. These $M_{V}$ are doubtful because of uncertainty of their mass.

Let us turn first to some statistical features of our results that bear on the population types of blue stars at high latitudes, and the runaway, or high-velocity Population I stars. The mean apparent magnitude $\left\langle m_{V}\right\rangle=10.6$; the uncorrected moduli $\langle m-M\rangle=13$, from our approximate spectroscopic luminosities. The normal stars in the halo are at a mean distance near $4 \mathrm{kpc}$. Given plausible nuclear lifetimes for massive Population I stars this is not an impossible maximum excursion from their places of origin, near the galactic

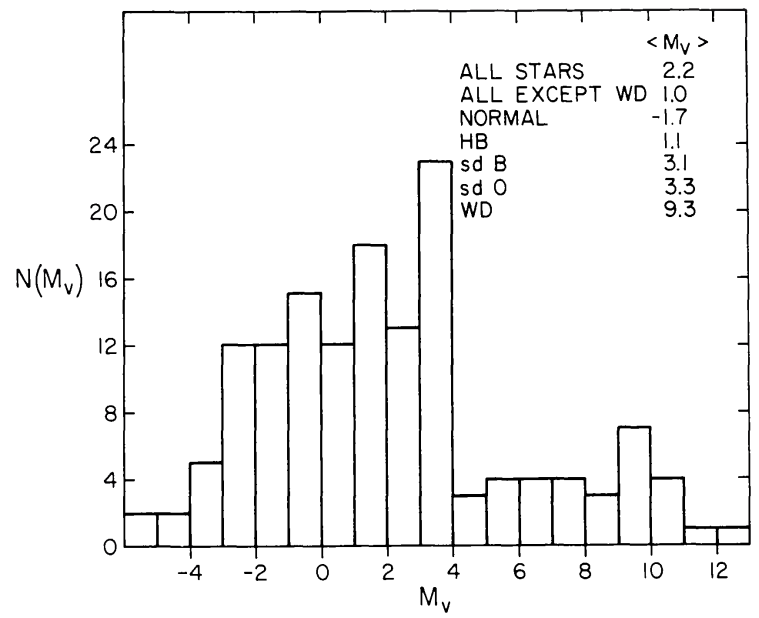

Fig. 9.-Frequency $N\left(M_{V}\right)$ of stars of a given $M_{V}$ for all FB stars (except composite). The $\left\langle M_{V}\right\rangle$ for various groups is tabulated.

plane, but the initial $W$-component, $W(0)$, needed ranges from 60 to $260 \mathrm{~km} \mathrm{~s}^{-1}$, (or to $180 \mathrm{~km} \mathrm{~s}^{-1}$ for the truly normal stars). A most serious problem then concerns the ejection of so many runaway stars, say, from Blaauw's (1961) (supernova, binary-star)-mechanism. Rodgers (1971) has already discussed a similar difficulty raised by the large number of apparently normal A stars found near the South Galactic pole, at smaller $\langle z\rangle$ coordinates.

For most, the radial velocities do indicate large space motion. The radial-velocity data is found in table A7. The distant group includes many apparently normal Population I stars, on the basis of the appearance of their spectra, e.g., normal C II, Mg II, Ca II, and other line strengths, and rotational broadening. Proper motions are too small to give $U, V, W$ components for most stars, given both their large errors, up to \pm 0 ".015, and the large distances of the stars. At a mean paral$\operatorname{lax}<0$. 001 , kinematics from the $W$-component, from the radial velocity alone, are far more accurate than from $U$ or $V$. These require motions, including correction to an absolute system, with an accuracy of better than 0 ".003. A brief discussion of reliable $U, V, W$ 's will be found later. If runaway stars have nuclear lifetimes of the order of their transit time to large $z$, they will be red giants or blue stars above the ZAMS even on their first return to the galactic plane.

The metallic-line strengths in the "normal" stars are much as expected, with a few slightly weak-lined objects included. Measured were C II, Ca II, and $\mathrm{Mg}$ II, but visual inspection showed O II, Si II, Fe II, Ti II, Si III, and other elements on the higher-dispersion spectra. The most interesting results are for He I lines, which we show in figures $10 \mathrm{a}$ and $10 \mathrm{~b}$. The triplet-singlet ratio depends on the helium abundance, through curve-ofgrowth effects; we combine both in one plot, using a different scale for $\lambda 4471$ and $\lambda 4388$. The curves drawn through the data for the normal stars of table A1 will be used later for comparison with other kinds of halo stars. However, individual stars deviate upward in figure 10 by large amounts, e.g., FB 46, FB 50, FB 135, 
FB 168, and FB 183. These seem to be helium enriched, probably by a considerable factor. FB 168 is a wellknown He-rich star with almost no hydrogen lines. A few are significantly below the mean curve, for example, FB 136, FB 158, FB 162. FB 162 was found by Traving to resemble Barnard 29, in M13, being a highly evolved star of low mass. FB 158 is probably similar (Danziger and Jura 1970). As we will see, there are reasons to doubt the completely normal nature of an appreciable fraction of the "normal" stars in table A1; those we suspect of various peculiarities have $M_{V}$ marked with an asterisk in table A1, and discussed in detail below.

It is of relevance to note the analysis by Lester (1972) of the rotating Population I, helium-rich star HD 37017, a member of a spectroscopic binary. He finds helium lines $W(4388)=1.40 \AA, W(4471)=2.14 \AA$, high in figure 10 , but not outstandingly so when compared to some of our strong-helium, normal stars. From the data he gives we derive $\theta=0.25$ and $\log g=3.94$, quite close to the main sequence. His detailed analysis gives $\mathrm{He} / \mathrm{H}$ twice its normal value, $\theta=0.24, \log g=4.4-4.5$. The small $\mathrm{He}$ overabundance has not drastically altered the results from either Lester's exact analysis or our rough one; the two agree fairly well. Study of the effect of larger $\mathrm{He} / \mathrm{H}$ ratios, by Böhm-Vitense (1968), is unfortunately limited to $\theta \geq 0.39$. At $\theta=0.39$, the $\mathrm{H} \gamma$ profiles are slightly broadened (because of the higher gas pressures) until the $\mathrm{H} / \mathrm{He}$ ratio is decreased by over $10^{2}$. At $\log g=4.5, \theta=0.39$, and a reduction in $\mathrm{H} / \mathrm{He}$ by $10^{3}$, for example, $D(0.2)$ drops from 23 to $14 \AA$; from figure 3, we would have deduced a decrease of $\log g$ from 4.55 to 3.56 , i.e., we would have thought the helium-rich star 2.5 mag too bright. It would be interesting to test such effects at higher temperature. The weakening of the hydrogen lines, and the disappearance of the Balmer jump would have important consequences in any method of analysis.

\section{b) Are the Normal Stars Normal?}

Some of the normal stars are among the apparently brighter of the FB stars. We may expect even quite young massive stars to be found nearby, in the galactic polar caps. Other criteria of "normal" are stigmata of young Population I stars near the Sun. We defined the normal stars in table A1 as those with surface gravities on or above the ZAMS, and with normal line strengths of helium and the abundant elements. But an additional criterion can be the rotation. No HBA or sdG star so far studied at high dispersion has shown appreciable rotation. At the $\mathrm{Pe}\left(38 \AA \mathrm{mm}^{-1}\right)$ dispersion $v$ sin $i>60$
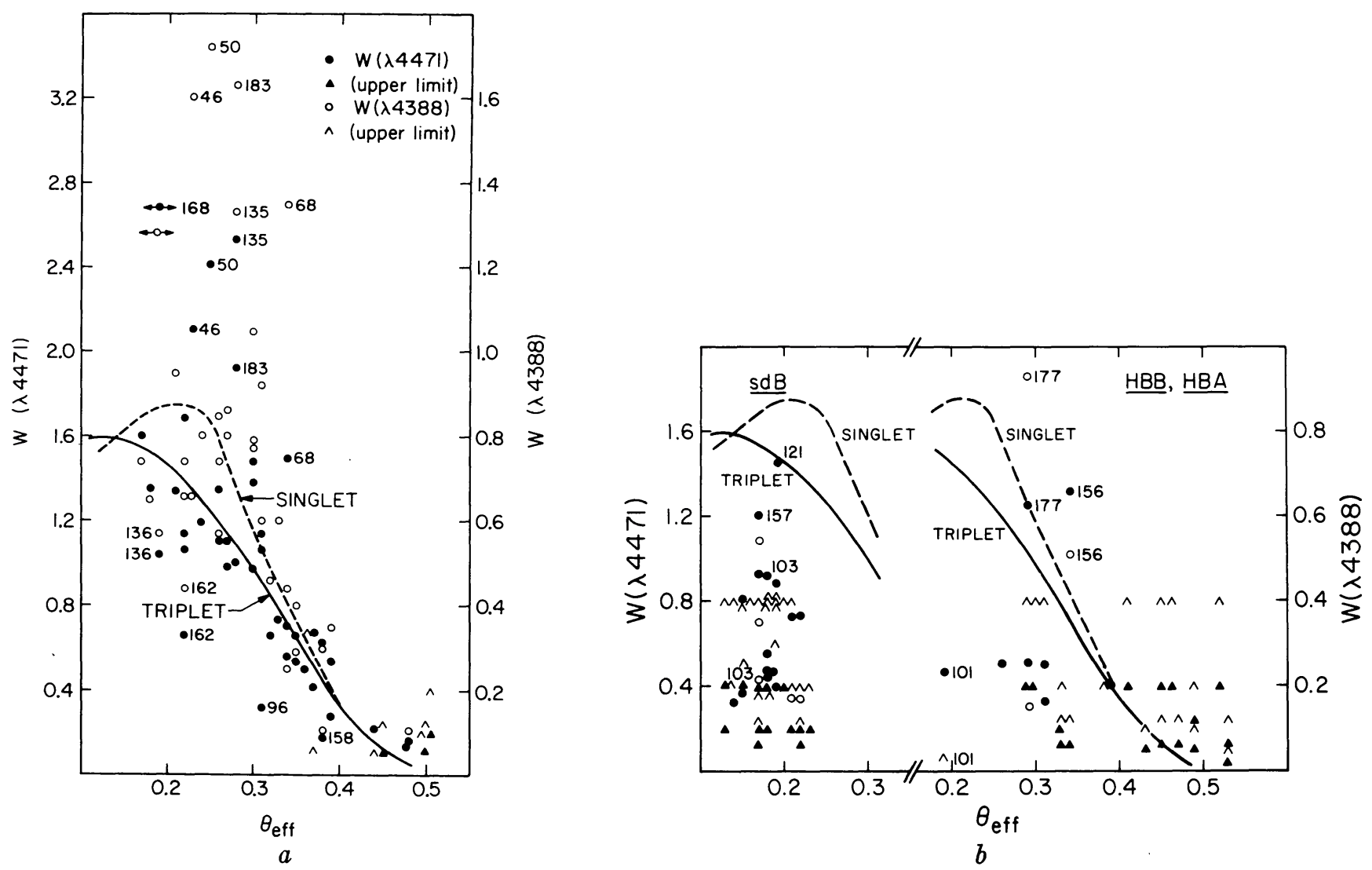

Fig. 10a.-The equivalent widths of the triplet ( $\lambda 4471$ ) (filled circle) and singlet $(\lambda 4388)$ (open circle) are shown for normal stars (table A1). Individual numbers given are FB serial numbers. The mean relation for the triplet is a solid line and for the singlet a dashed line. Filled triangles, upper limits for $W(4471)$; carets, upper limits for $W(4388)$.

Fig. 10b.-We display He I line strengths for HBB and HBA stars, and separately for sdB stars; symbols as in fig. 10a. The $(W, \theta)$ relation for normal stars is sketched. 
$\mathrm{km} \mathrm{s}^{-1}$ is detectable, with proportionally lower limits at the Pd and Pc scales (available for many of these bright FB stars). In addition, radial velocities help distinguish the various populations.

To isolate those stars which may not be normal, we carefully reinvestigated the data, and predicted from line intensities whether the star should be a normal (even if a high-velocity) member of Population I. A tentative prediction that the star might be sufficiently peculiar so as not to be called normal was based on excessively strong or weak helium lines and weaker than expected $\lambda \lambda 4267,4481$ (and $\lambda 3933$ for cooler stars). About 20 stars were suspected of being abnormal, based on the data for 50 stars in table A1. Of these, visual inspection showed 13 as having excessively strong or weak $\mathrm{He}$ I, weak $\mathrm{C}$ II, $\mathrm{Mg}$ II, or $\mathrm{Ca}$ II lines, or excessively small or undetectable rotation. The latter phenomenon was particularly conspicuous in the weak He I stars. Insofar as the term " $\lambda$ Boo star" is a meaningful one, for these hotter objects, it was applicable to most of the 13 slightly abnormal stars. In addition, revealed only during the visual inspection, FB 180 was noted to have a background of sharp metallic lines, largely $\mathrm{Si}$ II, Fe II, Ti II, with slightly anomalous $\mathrm{He}$ profiles. Thus it was a slightly peculiar B star, but almost certainly of Population I. Table A1 and inspection agreed in revealing 18 of 50 stars to have some peculiarity, not an unusual ratio among $B$ stars. But 23 of 50 are noted as "rotating" or "rapidly rotating". The sample is largely Population I in composition and rotation, with a small admixture of evolved halo stars of low mass (resembling those in globular clusters).

\section{c) Nuclear Ages and Kinematics of Normal Stars}

The method of analysis gives $\theta, g$ purely from photometry and line profiles. We have transformed them into luminosities by assuming main-sequence masses for the apparently normal stars. The stars often have been ascribed so high a luminosity that they are far from the galactic plane, at a $z$-coordinate which requires a very large initial $W(0)$, the velocity $W(z)$ at $z=0$. If we can neglect the $U, V$ components of a galactic orbit, it is possible to compute the time to reach $z$, with velocity $W(z)$, the residual velocity in the $z$-direction. The solar motion in the $W$ direction $\left(+6 \mathrm{~km} \mathrm{~s}^{-1}\right)$ can be subtracted from $\rho^{\prime} \sin b$, to derive the tabulated $\rho^{\prime}(z)$, the radial component in the $z$-direction. The Oort (1932, 1960) $K(z)$ has been kindly modified and extended by Maarten Schmidt for our computations, and the integrations performed numerically by Jay Elias. He provided $t(z, w)$, the time since the last crossing of the plane, the velocity $W(0)$ at crossing, and the full oscillation period, $P$, given in table 4 . Oscillation far from the plane may bring the stars to so large a height that the force exerted by the galactic center may dominate; the crossing times in such orbits are particularly sensitive to the unknown $U$ and $V$ components of the motion.

The expected nuclear lifetimes, $\tau$, can be derived from the following simplified model. From our observed data $g, \theta$ we derive, in fact, the $\mathrm{m} / L$ ratio,

$$
\mathfrak{m} / L=\alpha g \theta^{4} .
$$

Let us combine this value of $\mathrm{m} / L$ with the nuclear energy available. In the simplest model for hydrogen burning the integrated nuclear energy output, $\langle L\rangle \tau$, is $6 \times 10^{-3} X_{\mathrm{H}} q \beta(\mathrm{m}) \mathrm{m} c^{2}$, where $X_{\mathrm{H}}$ is the hydrogen content, $q$ is the ratio of the mass of the exhausted core of helium to $\mathfrak{m}$, the mass of the star; the correction $\beta(\mathfrak{m})$ allows for variation of $q$ with mass (increasing in massive stars) and for energy from the contraction of the core and early stages of shell burning as the star moves upward and to the right above the ZAMS. If we take $\beta(\mathfrak{m})=1$, and use $X_{\mathrm{H}} q \beta(\mathfrak{m})=0.12$, we obtain a lower limit to $\tau$. The true nuclear evolutionary time, $\tau_{E}$, near and above the ZAMS, with the object still a B star, is unlikely to exceed $\tau_{E} / \tau=2$. Combining the constant of proportionality in equation (10), with the nuclear-energy-yield constants, and eliminating $\mathfrak{m} / L$, gives $\tau$ in years as

$$
\begin{aligned}
\tau & =\frac{\beta(\mathfrak{m}) X_{\mathrm{H}} q 6 \times 10^{-3} c^{2} g \theta^{4}}{3.156 \times 10^{7} 4 \pi \sigma G(5040)^{4}} \\
& =6.69 \times 10^{5} \beta(\mathfrak{m}) g \theta^{4} .
\end{aligned}
$$

Then from Iben's (1967a) résumé of time scales for ZAMS life and early evolution, the $\beta(\mathfrak{m})$ factor was evaluated; it decreases from $1.30\left(15 \mathrm{~m}_{\odot}\right)$ to $0.72\left(2.25 \mathrm{~m}_{\odot}\right)$. Most of the lifetime is near the ZAMS; the shell source moves the star to $\theta>0.5$ rapidly, and helium burning in the core occurs only for the most massive stars before they also become too cool. We used the observed $L / \mathrm{m}$ value (from $g \theta^{4}$ ), but the evolutionary tracks show that $L / \mathfrak{m}$ rises by about a factor of 2 during early evolution. We did not allow for this in evaluating $\beta(\mathfrak{m})$; it would shorten the lifetimes further. However, it is interesting to note that most of the surface gravities observed are less than the ZAMS value.

From equation (11) with these corrections the nuclear time scales in table 4 are obtained. From the travel time $t_{z, W}$ to the star's present $z$ and $\rho^{\prime}(z)$, we isolate those stars for which $t_{z, w} \gg \tau$, and give remarks concerning spectroscopic peculiarities, if any exist. Asterisks denote the size of this discrepancy. A large number, 11 , have $t(z, W)>3 \tau$; of these, six are either evolved giants of Population II, or low-mass binaries, or have stronger than normal helium and should be deleted.

The kinematical behavior of these stars is, however, quite strange. For only seven is the period, $P$, of a full vertical oscillation (from the maximum $z$ through the galactic plane and back to the maximum $z$ ) less than or equal to the nuclear lifetime, $\tau$. The stars are not kinematically relaxed; they are apparently observed soon after formation and ejection. The sample is, of course, highly selected. We have no information on the number at low velocities; $W(0)$, the speed at crossing the galactic plane, without regard to sign, has a mean value of $119 \mathrm{~km} \mathrm{~s}^{-1}$, with a spread of $\pm 40 \mathrm{~km} \mathrm{~s}^{-1}$. These statistics essentially reflect our distribution in the $z$-coordinate. The mean $W(0)$ is larger than the observed dispersion in $\rho^{\prime}(z)$ of $66 \mathrm{~km} \mathrm{~s}^{-1}$, but stars with 
TABLE 4

KINEMATICS OF NORMAL STARS FROM TABLE AI

NUCLEAR LIFETIMES AND TIMES SINCE CROSSING THE GALACTIC PLANE

\begin{tabular}{|c|c|c|c|c|c|c|c|c|c|}
\hline $\mathrm{FB}$ & $\log \tau$ & $\log t(z, w)$ & $\log P$ & $\mathrm{z}_{\mathrm{kpc}}$ & $\rho^{\prime}(z)$ & $\mathrm{W}(0)$ & $t(z, W)>\tau$ & Pop. & Remarks \\
\hline 5 & 8.13 & 7.51 & 8.02 & 0.75 & -12 & 32 & ... & $\ldots$ & \\
\hline 7 & 7.30 & 7.58 & 8.49 & 5.07 & +83 & 175 & $\star$ & I & He-rich? \\
\hline 16 & 8.07 & 7.52 & 8.07 & 0.96 & -13 & 59 & ... & ... & \\
\hline 17 & 8.18 & 7.64 & 8.23 & 2.37 & -4 & 102 & $\ldots$ & $\ldots$ & \\
\hline 22 & 8.21 & 7.64 & 8.24 & 2.47 & -1 & 104 & $\ldots$ & ... & \\
\hline 23 & 8.16 & 7.62 & 8.18 & 1.74 & -17 & 86 & $\ldots$ & $\ldots$ & \\
\hline 25 & 7.95 & 7.74 & 8.31 & 3.48 & -10 & 127 & $\ldots$ & $\ldots$ & \\
\hline 30 & 7.97 & 7.42 & 8.10 & 1.14 & +12 & 65 & $\ldots$ & ... & \\
\hline 37 & $(7.51)$ & $(7.68)$ & $(8.29)$ & $(3.18)$ & +5 & 121 & $\star$ & I & $\begin{array}{l}\text { He, C slightly strong. } \\
\text { Evolved? }\end{array}$ \\
\hline 40 & $(6.61)$ & $(8.11)$ & $(8.68)$ & $(11.52)$ & -13 & 130 & $\star \star \star *$ & I & He, C rich. Evolved. \\
\hline 46 & 7.59 & $\cdots$ & $\cdots$ & 0.03 & -5 & $\cdots$ & $\cdots$ & $\cdots$ & $\begin{array}{l}\text { He strong; velocity } \\
\text { variable? }\end{array}$ \\
\hline 50 & 8.00 & 7.57 & 7.86 & 0.99 & -17 & 61 & ... & $\ldots$ & He strong \\
\hline 54 & 6.98 & 7.85 & 8.36 & 4.02 & -33 & 141 & $\star \star \star *$ & I & Normal \\
\hline 63 & 7.25 & 7.58 & 8.30 & 3.18 & +33 & 125 & $\star$ & I & Normal \\
\hline 65 & 8.07 & 7.45 & 8.19 & 1.81 & +26 & 90 & ... & ... & \\
\hline 66 & 7.45 & 7.58 & 8.42 & 4.32 & +64 & 156 & $\star$ & I & Normal \\
\hline 68 & 7.75 & 8.00 & 8.60 & 9.25 & +160 & 198 & * & $I ?$ & $\begin{array}{l}\text { Small rotation; data } \\
\text { only fair. }\end{array}$ \\
\hline 72 & 8.15 & 7.60 & 8.20 & 1.59 & +111 & 80 & ... & I & \\
\hline 74 & 8.08 & 7.85 & 8.53 & 7.31 & +21 & 182 & $\cdots$ & ... & \\
\hline 76 & 7.13 & 7.71 & 8.31 & 3.47 & +211 & 127 & $\star \star$ & I & Normal \\
\hline 79 & 6.76 & 7.99 & 8.69 & 11.42 & +34 & 216 & 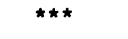 & I? & Evolved, low mass? \\
\hline 80 & 8.11 & 7.44 & 8.14 & 1.39 & +17 & 75 & ... & ... & \\
\hline 83 & 7.46 & 7.73 & 8.42 & 5.10 & +26 & 157 & $\star$ & I & Normal \\
\hline 89 & $(6.86)$ & $(8.23)$ & $\ldots$ & $(19.16)$ & -7 & $\ldots$ & $\star \star \star *$ & I & Evolved binary low mass. \\
\hline 92 & 7.81 & 7.53 & 8.15 & 1.75 & +9 & 85 & $\cdots$ & .. & \\
\hline 96 & 7.59 & 7.40 & 8.35 & 2.86 & +78 & 138 & ... & I & Possible Si star. \\
\hline 97 & 7.30 & 7.76 & 8.49 & 6.21 & +38 & 173 & $\star \star$ & I & Normal \\
\hline 98 & 8.20 & 8.12 & 8.46 & 2.03 & -138 & 166 & ... & I & Normal \\
\hline 104 & 7.70 & 7.92 & 8.30 & 1.70 & -91 & 123 & $\star$ & I? & slightly weak lines. \\
\hline 108 & 8.67 & 7.61 & 8.29 & 3.08 & +20 & 120 & $\cdots$ & ... & \\
\hline 113 & 8.09 & 7.69 & 8.25 & 2.57 & -13 & 108 & $\cdots$ & $\cdots$ & \\
\hline 118 & 8.84 & 7.84 & 8.28 & 2.48 & -57 & 119 & $\cdots$ & $\cdots$ & \\
\hline 119 & 8.57 & 7.77 & 8.32 & 3.53 & -18 & 129 & ... & $\cdots$ & \\
\hline 135 & 8.01 & 7.20 & 8.49 & 2.57 & +136 & 172 & $\ldots$ & $\ldots$ & He strong; evolved? \\
\hline 136 & $(7.46)$ & $(8.41)$ & $(9.01)$ & $(24.40)$ & +77 & $(261)$ & $\star \star \star$ & II? & He strong, sharp;poor data. \\
\hline 140 & 7.41 & 7.93 & 8.48 & 6.24 & -22 & 170 & $\star \star$ & I & Normal \\
\hline 141 & 7.11 & 7.99 & 8.35 & 3.99 & +17 & 138 & ... & ... & \\
\hline 146 & 7.16 & 7.09 & 8.66 & 2.46 & +181 & 209 & $\ldots$ & I & He slightly strong. \\
\hline 152 & 8.28 & 8.00 & 8.40 & 3.36 & -88 & 152 & $\cdots$ & $\cdots$ & \\
\hline 153 & 7.31 & 7.74 & 8.37 & 4.48 & +9 & 145 & * & I & Normal; velocity variable. \\
\hline 158 & $(6.76)$ & $(7.88)$ & $(8.36)$ & $(3.77)$ & -49 & 141 & $\star \star \star$ & II & $\begin{array}{l}\text { Globular cluster giant; } \\
\text { velocity variable. }\end{array}$ \\
\hline 159 & 8.15 & 7.77 & 8.28 & 2.93 & -28 & 118 & $\cdots$ & I & \\
\hline 162 & $(5.61)$ & $\cdots$ & $\cdots$ & $(39.31)$ & -84 & $\cdots$ & $\star \star \star *$ & II & Globular clușter giant. \\
\hline 164 & 6.87 & 7.67 & 8.27 & 2.98 & +2 & 116 & $\star \star \star$ & $\mathbf{I}$ & Normal \\
\hline 168 & $\cdots$ & $\cdots$ & $\cdots$ & $\cdots$ & +6 & $\cdots$ & $\cdots$ & $?$ & He-rich \\
\hline 174 & 8.12 & 7.42 & 8.02 & 0.74 & -9 & 48 & ... & -.. & \\
\hline 180 & 7.67 & 7.69 & 8.17 & 1.57 & -31 & 85 & $\cdots$ & I & silicon star \\
\hline 181 & 7.79 & 7.67 & 8.19 & 1.90 & -21 & 91 & ... & $\mathbf{I}$ & \\
\hline 183 & 7.87 & 7.18 & 8.27 & 1.56 & +84 & 115 & $\cdots$ & I & He strong \\
\hline 187 & 7.55 & 7.39 & 8.09 & 1.08 & +16 & 64 & $\cdots$ & $\cdots$ & Low rotation \\
\hline
\end{tabular}


small $W(0)$ do not reach high $z$, and would not have been included in the FB sample. Inspection of the residuals $\rho^{\prime}(z)-\left\langle\rho^{\prime}(z)\right\rangle$ shows a single Gaussian to be a poor fit. The $\chi^{2}$ test gives a 0.1 percent probability to the observed distribution. If only four highly deviant residuals $\geq 100 \mathrm{~km} \mathrm{~s}^{-1}$ are arbitrarily excluded, the $\chi^{2}$ for the artificially truncated distribution of 38 data points has a probability of 40 percent. There exist, apparently, a few genuine runaway stars plus a normal population with $\left\langle\rho^{\prime}(z)\right\rangle=+4.5 \pm 6.9 \mathrm{~km} \mathrm{~s}^{-1}$, with a dispersion of $\pm 42.3 \mathrm{~km} \mathrm{~s}^{-1}$ in $W(z)$. The z-component dispersion of the normal" FB stars is then not too different from that of subdwarf B stars of the halo; this statement is a fairly close consequence of the data. It is invalidated only if our normal stars are almost all, in fact, Population II, which seems quite implausible.

Given the fundamental problem that far too many, hot, high-velocity, apparently normal stars exist, are there any suggestive clues for stellar evolution to be found in the data of table 4 ? A reexamination of the nuclear lifetimes is in order. It would be interesting to search for the possible existence of as yet unexplored, short-lived stages. For a speculative example, let mass loss occur, with helium flashing and mixing. A hot star with surface rotation might be produced if the stellar core had retained a relatively high angular momentum per unit mass. Its mass would be less than its initial value, and it would not obey the mass-luminosity relation. Its lifetime as a hot, bright star would be short. Our data give $g \theta^{4}$, i.e., the present value of $\mathrm{m}_{0} / L_{0}$ (eq. [10]). We want $\tau_{i}$, the initial ZAMS life. Let us assume that $\mathfrak{m}_{0}=\epsilon \mathfrak{m}_{i}$ and $L_{0}=\gamma L_{i}$. From $\mathfrak{m}_{0} / L_{0}=\alpha g \theta^{4}$, $\mathfrak{m}_{i} / L_{i}=(\alpha \gamma / \epsilon) g \theta^{4}$. The total nuclear energy consumed on or near the ZAMS gives a $\tau_{i}$ (eq. [11]) of

$$
\tau_{i}=(\gamma / \dot{\epsilon}) \tau=6.69 \times 10^{5} \beta\left(\mathrm{m}_{0} / \epsilon\right)(\gamma / \epsilon) g \theta^{4} .
$$

The total life of the star is of the order of $\tau_{t}=\tau_{i}+\tau$; lacking details of the evolution, it will be sufficient within a factor of 2 to adopt $\beta\left(\mathrm{m}_{0} / \epsilon\right)=\beta\left(\mathrm{m}_{0}\right)=\beta\left(\mathfrak{m}_{i}\right)$. Then

$$
\tau_{t}=\tau(1+\gamma / \epsilon) .
$$

As an example, for a horizontal-branch star descended from a star of solar mass, $\gamma \simeq 50, \epsilon \simeq 0.66$, and $\tau_{t}=$ $77 \tau$. Since the horizontal-branch stars have a mean log $g \theta^{4}=2.35$, we find $\tau=1.50 \times 10^{8}$ years at the current luminosity, and $\tau_{t}=1.15 \times 10^{10}$ years in total, a quite satisfactory approximation.

Table 4 contains 21 stars marked as strongly suggested to have $t(z, W) \gg \tau$. A few are clearly Population II, i.e., not normal. The 18 with complete data have a $\langle\log P / \tau\rangle=1.24$; omitting Population II or other suspicious stars (possible binaries) we have 13 stars with $\langle\log P / \tau\rangle=1.08$. Clearly any choice of a value of $P / \tau$ to be labeled "suspicious" is subjective. All the stars could have been formed in a single burst at a recent time; none could have existed in their present state since the beginning of star formation. If a steady state is thought necessary, $\log P / \tau$ should be negative; if an interval of the order of $10 P$ is desirable, $\tau_{t}$ should be $\sim 2 \times 10^{9}$ years. With this guess, $\log P / \tau_{t}=-1.0$, $\log \tau_{t} / \tau=+2.08$ which leads to $\gamma / \epsilon=119$, for the suspicious cases in table 4 . Other stars for which log $P / \tau \simeq 0$ require less extreme values of $\gamma / \epsilon$. We require that perhaps half of the stars now be found in a shortlived evolutionary stage of high temperature and at about 100 times their initial luminosity. In Population II, such evolutionary stages exist; we can mention Barnard 29 in the globular cluster M13, for which $\gamma=400$. The luminosity, $M_{V}=-1.7, \log L / L_{\odot}=$ 3.40; from its $\log g \theta^{4}=+0.23$ (Stoeckly and Greenstein 1968) we would derive $\tau$ of only $10^{6}$ years, but $\tau_{t}$ must be near $10^{10}$ years. The star has lost, probably, about 80 percent of its initial mass. Certain stars of table 4 may be suspected of similar behavior, because $P / \tau>$ 20. FB 37 and FB 40 are carbon and helium rich, FB 54 has weak C II and Mg II lines, FB 89 (and possibly FB 79) is a low-mass binary, FB 146 has strong helium lines, FB 136 (He weak), and FB 158, FB 162 are related to the globular-cluster type giants. A few of the stars with weak metals, FB 54, FB 63, FB 76, have moderately large $P / \tau$. They remind us again of the $\lambda$ Bootis stars, which Baschek and Searle (1969) find to be kinematically normal, rotating, Population I mainsequence stars with low surface metal abundances.

If some stars are temporarily overluminous, for an as-yet-unknown reason, we used too high a mass in determining their luminosities (having invoked the normal mass-luminosity law). We may bring them closer to the galactic plane by arbitrarily reducing the masses, and thus shorten their oscillation periods. However, only modest gains in the discrepant values of $P / \tau$ are possible. If evolution is at constant $L$, i.e., $\gamma=1$, but at a different mass, the luminosity is changed only in proportion to the mass. Few of our normal stars have estimated masses greater than $20 \mathrm{~m}_{\odot}$, and unless they are in the unknown evolutionary stages mentioned above, they are unlikely to have masses less than $4 \mathfrak{m}_{\odot}$. From equation (5), we expect a reduction of $\Delta M_{V} \lesssim$ +1.75 , and of $\log z$ by $\leq-0.35$, i.e., by about a factor of 2 . The period of the $z$-oscillation varies roughly as $z^{0.54}$ from 1 to $10 \mathrm{kpc}$. The reduction in the $\log P$ given in table 4 is roughly then -0.19 , quite insufficient to affect the discrepant $\tau / P$ values significantly. The effect of the temporary high $L / \mathfrak{m}$ assumed to exist is largely the increase in $\tau_{t} / \tau$, as discussed above.

\section{d) Comparison With Other Observers}

Sargent and Searle (1968; hereafter referred to as SS) studied 22 Feige stars of our FB list on $48 \AA \mathrm{mm}^{-1}$ Lick Observatory spectra. They measured hydrogenline profiles and Balmer discontinuities and had spectrophotometric scans of the continua of some. From the early Mihalas (1965) models, with some extrapolation, they estimated $\log g, \theta$ and then classified the stars into descriptive groups. When we compare our normal-star classifications and theirs, we find excellent agreement. The normal FB stars FB 22, FB 25, FB 72, FB 80, FB 135, and FB 153 are classified by $\mathrm{SS}$ as main sequence; FB 119 is classified B8 III, in agreement with our quite low surface gravity, $\log g=3.2$. They classify FB 83, FB 113 as Bw, i.e., weak-lined stars, a descrip- 
tion otherwise reserved for our horizontal-branch stars. Inspection shows that FB 83 is rotating and has normal Mg II, He I, and C II lines, while FB 113, a late B star also rotating, seems to be somewhat weak-lined (our so-called hot analog of the $\lambda$ Boo phenomenon). Not all of the Bw stars in SS need belong to the halo population.

The normal stars common to the SS and FB lists have low radial velocities, except for two (FB 72, FB 135) measured only on $190 \AA \mathrm{mm}^{-1}$ dispersion. Seven of these have reliable $M_{V}$ in table A1 with a mean, from spectroscopic techniques, of -0.9 . The proper motions are very small, but as reliable as any we will have; the mean $\left\langle m_{V}\right\rangle=11.13$, the mean total absolute proper motion from Luyten (1969) is $\langle\mu\rangle=0$.0128 \pm 0 . 0031 . The radial-velocity dispersion of these stars is $66 \mathrm{~km} \mathrm{~s}^{-1}$. Using the method of reduced-proper motions, averaging $\langle\mu\rangle$ and with $\langle T\rangle=0.5 \pi \sigma_{W}$, we derive ${ }^{2}\left\langle M_{V}\right\rangle=0$. Using individual values of $H=m+5 \log \mu$ and averaging, in contrast, $\langle\log \mu\rangle$, we obtain $\left\langle M_{V}\right\rangle=-0.6$. If we correct the observed $\mu$ for errors of proper motion by assuming an average error of \pm 0 ". 005 in $\mu_{\alpha}$ and $\mu_{\delta}$, i.e., \pm 0.007 in $\mu$, the random $\langle\mu\rangle$ is reduced to $0{ }^{\prime \prime} 0107$, which leads to $\left\langle M_{V}\right\rangle=-0.4$. Within the errors of method and observation, the normal FB stars in the SS list are correctly classified, and are, in fact, clearly far brighter than the horizontal-branch stars.

This evidence is important when comparing with the work of Newell (1969). He used narrow-band colors, some data from SS, and image-tube spectroscopy of hydrogen lines. In his thesis study of field stars he gives results for the seven Feige stars observed by SS. He placed them in his group $\mathrm{F}$. The latter group is near the intersection in the $(\log g, \theta)$-plane of the main sequence

2 The Appendix, in discussion leading to equation (A3), suggests methods for allowance for the ellipsoidal velocity distribution. For these same normal stars the luminosities become $\left\langle M_{V}\right\rangle=$ -1.2 or -1.8 (eq. [A3]).

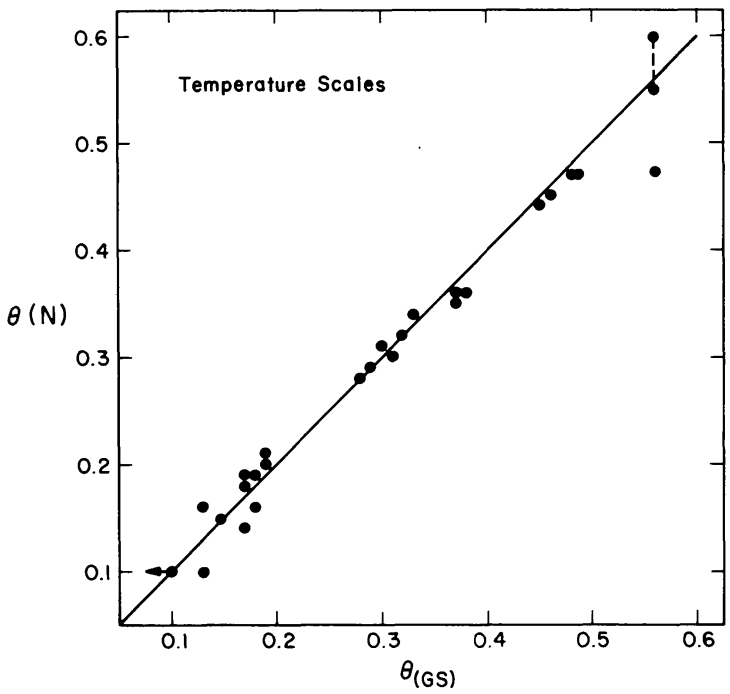

$a$ and the halo-horizontal-branch locus. Such an ambiguity arises if stars of different masses have the same $\log g$ and $\theta$, but this is quite consistent with the RussellVogt theorem. Newell linked the stars of group F with the $\mathrm{HB}$ stars, and ascribed them low mass; we have shown above that all are spectroscopically normal stars. SS call most of them main-sequence stars, as do we. In addition to stars discussed above, FB 22 has somewhat weak $\mathrm{He} \mathrm{I}$ and $\mathrm{Mg}$ II lines but rotates rapidly; FB 25 and FB 80 have normal line strengths and high rotation; FB 40 and FB 135 (observed only at $190 \AA \mathrm{mm}^{-1}$ ) have normal or strong $\mathrm{He}$ I; FB 153 may be a velocity variable and has normal line strengths and rotation. Unless we are willing to omit all evidence from line strengths and surface rotation, it seems that most of Newell's group F is not related to the HB stars. (Of course, relatively metal-rich $\mathrm{HB}$ stars exist, but most have low surface helium.) Two of Newell's stars, FB 113 and FB 119 are in our normal star list. The former, as noted by SS, is weak lined, but probably rotates; FB 119 has rapid rotation and normal line strength.

Figure $11 a$ compares temperatures determined often in quite different ways. There is no scale error; certain ambiguities exist near $\theta=0.5$ in Newell's method, and produce some scatter. The $\log g$ agree well, with $\log g$ (Newell) $-\log g(\mathrm{GS})^{3}=0.14$, in figure $11 b$, in the range $3.0 \leq \log g \leq 4.4$. The scatter at large $g$ is not unreasonable, and arises from model changes, omission of $\mathrm{He} \mathrm{II}$, and the increasing effect of errors in $\theta$ on $\log g$ at high $\theta$ (where most high- $g$ stars are found).

After our above analysis was completed, Newell (1973) revised his thesis, changing some of his classification patterns. He supplemented his own Australian observations with data on $50 \mathrm{FB}$ stars taken from our preliminary results published by Greenstein (1971). His tables and figures include cooler halo stars, data

\footnotetext{
${ }^{3} \mathrm{GS}=$ Greenstein and Sargent (this paper) throughout.
}

FIG. 11a.-The comparison of our temperature scale $\theta(\mathrm{GS})$ with Newell's $\theta(\mathrm{N})$.

FIG. 11b.-Comparison of surface gravities, GS and N. 
from others on white dwarfs, planetary nuclei, and globular-cluster stars. It would be pointless now to compare our data or conclusions with his, especially since about half of the data on stars with $\theta<0.30$ in his summary table 7 come from our work. We cover a more restricted range of $\theta$ than he, but make more use of actual spectra, of which he had only few. The difference of substance is his inclusion, as a major type of halo star, of a group called HL, which in the mean has $\log g$ smaller by about 1.6 than do the horizontal-branch stars (his groups now called A, B, C, and D). Inspection of his results indicate that such stars would have $M_{b} \simeq$ -3.5 , if low mass, and be highly luminous if of normal main-sequence mass. Stars of our "normal" group seldom appear as bright (i.e., have as low gravity) as do some of Newell's HL stars; we note that his anomalous colors cause him to ascribe interstellar reddening $E(B-V)$ from 0.13 to $0.49 \mathrm{mag}$ to seven stars in his table 3; for only four of his HL stars is $E(B-V)$ as small as 0.03 . Most of these are 12th mag LB southern hemisphere stars, measured with Newell's narrow-band three-color system, and an interference $\mathrm{H} \gamma$ filter to give $W(\mathrm{H} \gamma)$; unfortunately, he had spectra of only one, LB 3116. The HL stars cannot be of type DA. It is possible that many of the HL stars are low-gravity members of our normal group since he so classifies HD 137569 (=FB 158) and $\pm 33^{\circ} 2642$ (=FB 162), which are certainly low-mass halo stars. Still another, $+10^{\circ} 2179$ is a very helium-rich star. About many of the stars in his table 7, for which he lists Greenstein (1971) as the reference, we differ in interpretation and suggest that the classification here be used. Some Newell HL stars lie not far from the Magellanic Clouds, in an area searched for blue stars by Luyten and Anderson (1959). They list LB 3404 as a possible emission patch, and Newell's chart shows it in a crowded field. Luminosities would be $M_{V}<-7$ if they are reddened Cloud members. Three stars (LB 3187, LB 3357 , LB 3384) are given reliable motions $>0$ ".010 by Luyten (1969) and should not be in the Clouds. Cloud members should not be included among our Galaxy's Population II high-luminosity stars in the halo. The statistics are surprising in that 20 percent of the 80 stars with $0.17 \leq \theta \leq 0.55$ classified by Newell are called HL, while he finds only one white dwarf. Of the 189 stars in this investigation there are only some 3 percent (see § VIII $b, c$ ) that seem likely to be halo high-luminosity stars. Most of our apparently bright stars are evolved Population I, we find. In addition, we have a larger fraction of white dwarfs.

\section{e) Helium in the Normal Stars}

The so-called normal stars have been the source of our data on expected strengths of $\mathrm{He}$ I singlet and triplet lines. Use of other lists of published equivalent widths would not help us define the [W( $\lambda), \theta]$-relation better, given systematic differences. Among these stars are several Population II objects, conspicuous for low surface gravity, and a number suspected of having abnormally strong He I lines. Since most have log $g$ less than do ZAMS stars, the gravity dependence of
He I line strengths operates in the wrong direction. Population I stars of high surface helium abundance have been studied recently by Osmer and Peterson (1973), and others have been known for some time. In addition, non-LTE atmospheres computed by Auer and Mihalas (1973) have gone far to explain in detail the helium singlet/triplet ratio, and to evaluate the gravity dependence of the helium lines in normal stars. At least for the equivalent widths and the ratio $\lambda \lambda 4388 / 4471$ the effects of non-LTE prove small, although large for lines in the red. The singlet/triplet ratio, $p$, has been used also as an indicator of the $\mathrm{He} / \mathrm{H}$ ratio, although, until now, no exact non-LTE computations for stars of low $\mathrm{He} / \mathrm{H}$ ratio have been published. Norris (1970) studied this topic in his thesis; Norris and Baschek (1970) find values between 0.5 and 0.9 , at normal helium abundance for the mean singlet/triplet ratio, and predict about 0.2 less for $\mathrm{He} / \mathrm{H}=0.05$. Table 5 gives a résumé of our singlet/triplet ratios for stars in which the He I spectrum is sufficiently well-developed to give a value of $p$. For 27 "normal" stars (table A1) observed at 9-38 $\mathrm{mm}^{-1}$ dispersions, $p=0.64 \pm 0.03$, while for nine HBB and sdB stars (tables A2, A3) mostly at lower dispersions, $p=0.36 \pm 0.06$. Somewhat expectedly, since sdO stars (table $\mathrm{A} 4$ ) are defined by the presence of $\mathrm{He}$ II, those 17 that have He I lines show $p=0.50 \pm$ 0.03 . This intermediate value would be an interesting check on hot-star He-rich models. Note that the nonLTE computations for normal $\mathrm{He} / \mathrm{H}$ abundances show an irregular dependence of $p$ on $\theta, g$, but predict values between 0.58 and 0.72 for $0.20 \leq \theta \leq 0.32,2.5 \leq \log$ $g \leq 4.0$. Over the small range of $\log g$ covered, $p$ decreases with increasing gravity and conceivably approaches the sdO star observed values, even with a high $\mathrm{He} / \mathrm{H}$ ratio. The low $p$ of 0.36 for the sdB stars clearly demonstrates their low surface helium abundance. In addition, such high-g stars lie far from the region where non-LTE effects can be important.

There is evidence for the existence of helium-rich, somewhat evolved, partially mixed, massive stars of Population I among our "normal stars." As indicated, a few had measured equivalent widths which were far above average for our normal stars. In fact, FB 37, FB 46, FB 135, and FB 183 have $\theta, g$ and $W(4471)$ similar to HD $60344,-46^{\circ} 4639,-69^{\circ} 1618$, and HR 5206. The means for the three FB stars were $\theta=0.25$,

TABLE 5

Helium Singlet-to-Triplet Ratios in FB Stars

\begin{tabular}{|c|c|c|c|}
\hline Group & $n$ & $p$ & m.e. \\
\hline 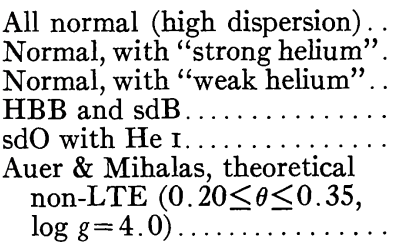 & $\begin{array}{r}27 \\
11 \\
7 \\
9 \\
17\end{array}$ & $\begin{array}{r}0.64 \\
0.64 \\
<0.33 \\
0.36 \\
0.50 \\
\\
\\
0.67\end{array}$ & $\begin{array}{l}0.03 \\
0.04 \\
0.08 \\
0.06 \\
0.03\end{array}$ \\
\hline
\end{tabular}

Note. $-p=W(4388) / W(4471)$. 
$\log g=4.2, W(4471)=2.06 \AA$, and $W(4267)=0.26$ $\AA$. For the Osmer and Peterson stars, these numbers are $\theta=0.20, \log g=4.12, W(4471)=2.38 \AA$ and $W(4267)=0.26 \AA$. Then our helium-rich objects can be explained as high-velocity Population I evolved stars. Our masses determined from equation (7) give a mean of $6.1 \mathrm{~m}_{\odot}$. The same phenomena suggested by Auer and Peterson for the evolution away from the ZAMS must be found in these high velocity stars, although our accuracy is far inferior. However, one interesting difference does appear since FB 135 and FB 183 have $\theta=0.28$. Auer and Peterson believe that the surface helium enrichment disappears for $\theta>0.24$, to be replaced by a helium surface deficiency in the typical Population I Bp or Si Ir $\lambda 4200$ stars at $\theta \gtrsim 0.28$. In addition, FB 37, FB 46, and FB 183 show rotation at $18 \AA \mathrm{mm}^{-1}$ dispersion, and only FB 135 has sharp lines. Auer and Peterson find little or no rotation ( $v$ sin $i<40 \mathrm{~km} \mathrm{~s}^{-1}$ ); they note, however, that the wellknown and similar He-rich star $\sigma$ Ori $\mathrm{E}$ has $v \sin i \simeq$ $150 \mathrm{~km} \mathrm{~s}^{-1}$. Lester (1972) found HD 37017 to be He rich at $\theta=0.24$, and rotating. Our $\theta$ 's determined from $Q$ may not be exact for He-rich stars, but the effect of $\mathrm{He} / \mathrm{H}$ variations on $D(0.2)$ cannot be determined from models available to us. Klinglesmith (1971) models show only small changes (near 5 percent) in $W(\mathrm{H} \gamma)$ at $\theta=$ $0.25, \log g=3$ or 4 , even for changes from $X_{\mathrm{H}}=0.14$ to 1.00 .

\section{HORIZONTAL-BRANCH AND HOT SUBDWARFS}

\section{a) General Methodology}

Appendix tables display the measurements and deduced parameters of 63 stars divided almost equally between what we call horizontal-branch $\mathrm{A}$ and $\mathrm{B}$ (table A2) and subdwarf B (table A3). ${ }^{4}$ We believe all these to be halo Population II stars related to the blue stars on the globular-cluster horizontal branches. We will call them, together with sdB and some sdO stars the extended horizontal branch (EHB). All but four of these were found by purely colorimetric methods (i.e., from the HZ, F, TON, TON-S, PHL, LNGP, and SBS surveys). The four Lowell proper-motion stars (GD) seem in no way different; some of the stars selected originally for color have comparable proper motions and $U, V, W$ components discussed below. Only southern globular clusters have HBA and HBB stars bright enough to be studied weith sufficient spectroscopic detail. Two faint hot stars in M13 lie in the same colormagnitude region as the sdB stars (Sargent and Searle, private communication). Since globular clusters have a variety of metal abundances we may expect variations in the strengths of lines of $\mathrm{Ca} I I, \mathrm{Mg} \mathrm{II}$, and $\mathrm{C} \mathrm{II}$; we have already mentioned the general low helium-line strength. We have combined globular clusters with different metal deficiencies in preparing the schematic horizontal branch of figure 6; similarly, the field EHB may have differing metal and surface helium content.

\footnotetext{
${ }^{4}$ N.B. - A misleading impression is given by the footnote to table A3, which refers only to FB 103 . Other objects marked with a dagger have $\mathrm{He}$ I relatively strong for $\mathrm{sdB}$ stars.
}

We must also be careful to search for any connection (or lack of connection) between the HB and sdB stars, given the infrequent occurrence of very hot sdB stars in globular clusters, and the large number in our table A3.

The HBB stars have surface gravities near to or above that on the main sequence. The HBA stars have lower surface gravity than the main-sequence stars; Sargent and Searle (1968) counted the number, $n$, of Balmer lines visible on the Lick spectra. We find some HBA or late HBB stars with $n>19$ although on $\mathrm{Pe}$ spectra we do not reach the region near the Balmer limit. Therefore, we first selected as prospective HB stars those with suitable values of $n$. There is an ambiguity where the $\mathrm{HB}$ star crosses the main sequence. Given the uncertainties in $\theta$ from colors, the range $0.28 \leq \theta \leq 0.42$ may be viewed as the difficult region. In addition, when $B-V>0.0$, the Balmer discontinuity is the same at $\theta>0.5$ and $\theta<0.5$, creating a second ambiguous region.

Having selected prospective HB stars by $n$ and color we found a number (most of which had to be omitted) with $\theta>0.56$. This is the limit of applicability of our techniques. For those between $0.50 \leq \theta \leq 0.56$ we used the relation between $\mathrm{Ca}$ II line strength and $\theta$ found by Rodgers (1971) for his high-latitude southern A stars. Such candidates are selected by having nearly normal $\mathrm{Mg}$ II line strength; $\mathrm{Mg} \Pi$ is only a slow function of $\theta$ in this range, and, if of normal strength, suggests that $\mathrm{Ca} / \mathrm{H}$ is also normal. In that case, $\lambda 3933$ determines $\theta$ and $D(0.2)$ yields $\log g$ without ambiguity. The $\mathrm{H}^{-} / \mathrm{H}$ opacity increase makes $D(0.2)$ a double-valued function of $\theta$, and sensitive to metal abundance. Of course, we are selecting the relatively high-metalcontent halo stars by this process, but we felt it important to have a few stars cooler than $\theta=0.5$ to ensure the fit of our HB stars to the clusters and to the results of narrow-band photometry such as Philip's (1968). At $\theta<0.5$ our technique works in a straightforward way until $\theta=0.43$, where errors of measurement may give $\log g$ values indistinguishable from main-sequence stars; by $\theta<0.28$ there is again a clear gap, with the hot $\mathrm{HBB}$ and sdB stars having log $g$ clearly greater than the main-sequence values. In the intermediate range we distinguish the HBB stars by inspection of the metallicline strengths. Some of the stars in table A2 have rather strong $\lambda 4481$ and are marked with asterisks. However, the $\mathrm{Mg}$ and $\mathrm{He} \mathrm{I}$ lines are still weaker than expected in main-sequence stars; inspection of the plates ( $\mathrm{Pd}$ and $\mathrm{Pe}$ for most) showed sharp lines (i.e., no large rotation). We may generalize by saying that all stars in table A2, if they have $g$ near the main-sequence value, have one or more of the indicators, $\mathrm{He} \mathrm{I,} \mathrm{C} \mathrm{II,} \mathrm{or} \mathrm{Mg} \mathrm{II,} \mathrm{weakened;}$ generally they have weak He I lines. Since $\theta, \log g$ are nearly the same, statistically, at the crossover region it was interesting to form the mean number of Balmer lines seen in normal stars (table A1) and in HB (table A2) in the range $0.28 \leq \theta \leq 0.43$. The result was $\langle n\rangle=15.2 \pm 0.5$ for 23 normal stars and $n=14.6 \pm$ 0.5 for 13 horizontal-branch stars. Thus, as expected, at comparable $\theta$ and $g$ the hydrogen spectrum does not depend on population type, The $\mathrm{H}^{-}$opacity, sensitive 
to the metal-to-hydrogen ratio, is negligible at such temperatures, and $g$ is only slightly changed by changes in the $\mathrm{He} / \mathrm{H}$ ratio. Thus the Paschen continuum slope and the Balmer discontinuity, which we use to determine $\theta$, and the hydrogen-line broadening, which determines log $g$ from $D(0.2)$, are closely the same. A few of the "normal" stars had low surface gravity, and are probably evolved Population I or even like globularcluster B stars. For example FB 158, log $g=2.4$, has $n>23$, confirming the suspicion that it is a highlatitude evolved giant. The mean $\log g=3.8$ for the normal stars was slightly less than the mean $\log g=4.0$ for the $\mathrm{HBB}$ stars in the overlap region.

The definition of sdB stars by Sargent and Searle (1968) has been followed in general; the sdB stars have such broad hydrogen lines that $n=10-12$. If $5.0 \leq \log$ $g<6.0$, the star is clearly a sdB star when no $\lambda 4686$ is seen. This difference from HBB stars is a description of Balmer line profiles purely. There are seven stars included in table A3 with $4.5 \leq \log g<5.0$. This lower gravity is, for many, within the observational errors, and $n$ is too small $(\sim 11)$ for the star to be called a HBB star. One such sdB star, FB 103 (Feige $66=$ Malmquist 299 ) included in table A3, is very peculiar. It has a weak $\lambda 4686$ line, and strong, sharp S III lines, so it may be described as a sdBp star. The hottest of the sdB stars are important as indicating the high-temperature limit of the surface helium deficiency. A few stars with $\mathrm{He}$ I lines slightly strong, but still far below the normal-star value, are indicated by daggers in table A3. The mean value of $n=11.0 \pm 0.2$ is higher than in white dwarfs, where $n$ never exceeds 10 .

One high-velocity sdB star, HD 205805, has been analyzed carefully on high-dispersion spectra by Baschek and Norris (1970) (BN). We include data for this star, listing it as FB 178 in table A3. It is too far south for the 200-inch telescope, but as one of the brightest B stars, it provides an invaluable check on our methods of analysis. Furthermore, it shows a weak $\mathrm{C}$ II line, which exists also in FB 9 (HD 4539), analyzed in a similar way by Baschek et al. (BSS, 1972), but not in the sdBp star FB 103 (F66) even at $9 \AA \mathrm{mm}^{-1}$ dispersion. The results given by the original authors for FB 9 and FB 103 are found in table 6 , in some cases with our simple reanalysis. It can be seen that the temperature scales are very similar, and that our log $g$ average is somewhat smaller $(\sim-0.2)$. This agreement may be viewed as excellent given the completely independent methods of analysis, and the different data and spectrographs used.

\section{b) Elemental Abundances}

Although it is generally to be assumed that the halo stars of large velocity dispersion are, like globular clusters, often metal poor, we can provide little direct evidence. Many of the stars deserve high-dispersion spectroscopy; it would be fascinating to see if there are metallic-abundance anomalies between the $\mathrm{sdB}$ and the HBB stars at the transition near $\theta=0.25$, which could possibly be related to the infrequency of globular-cluster sdB stars at higher temperatures. We study only one, FB 103, at high enough dispersion; it was apparently
TABLE 6

Comparison of Other Analyses of sdB Stars

\begin{tabular}{|c|c|c|c|c|}
\hline FB & Authors & $\theta$ & $\log g$ & $M_{V}$ \\
\hline 9. & BSS* & 0.20 & 5.4 & +3.6 \\
\hline 9. & GS† & 0.22 & 5.4 & +3.9 \\
\hline 13. & BN & 0.21 & 5.2 & +3.3 \\
\hline 69. & $\mathrm{BN}$ & 0.20 & 5.2 & +3.2 \\
\hline 101. & $\mathrm{BN}$ & 0.20 & 5.5 & +4.0 \\
\hline $13 \ldots \ldots$ & GS (table A3) & 0.19 & 5.5 & +3.9 \\
\hline $69 \ldots \ldots$ & GS (table A3) & 0.18 & 4.5: & +1.2 \\
\hline $101 \ldots \ldots$ & GS (table A2) & 0.19 & 4.8 & +2.1 \\
\hline 178. & $\mathrm{BN}^{*}$ & 0.19 & 5.0 & +2.6 \\
\hline $178 \ldots \ldots$ & GS $\dagger$ (data from BN) & 0.21 & 4.8 & +2.3 \\
\hline
\end{tabular}

*FB 9 has a logarithmic helium deficiency $[\mathrm{He} / \mathrm{H}]=-1.2$; $\mathrm{FB} 178$ has $[\mathrm{He} / \mathrm{H}]=-1.0$. The $[\mathrm{C} / \mathrm{H}]$ ratio is roughly normal.

$\dagger$ We give $\theta, g$ for FB 9 in table A3 by our method, using BSS data. We give $\theta, g$ for FB 178 by our method, using $B N$ data.

$\ddagger$ FB 13, FB 69, and FB 101 were roughly analyzed by BN using Lick data from SS.

metal and silicon deficient, but sulfur rich; FB 9 and FB 178, studied by $\mathrm{BN}$ and $\mathrm{BSS}$, have marginal $\mathrm{C}, \mathrm{O}$ deficiencies. The most promising line is $\lambda 4267$ of $\mathrm{C} \mathrm{II}$, which in normal stars is about $300 \mathrm{~m} \AA$ at $\theta=0.25$. We give upper limits near $200 \mathrm{~m} \AA$ for many hotter sdB stars, and for a few $<120 \mathrm{~m} \AA$, i.e., $\lambda 4267$ not seen at $38 \AA \mathrm{mm}^{-1}$. At $\theta$ near 0.35 normal stars have $W(4267)$ between 100 and $200 \mathrm{~m} \AA$ (although many have no 74267). This is the HBB temperature range; the stars are apparently brighter, and at $0.29 \leq \theta \leq 0.40$ we have five FB stars with coudé spectra. These either give $W(4267)$ or upper limits near $100 \mathrm{~mA}$. FB 137, which shows P II, is certainly hot enough, but still has $W(4267)<50 \mathrm{~m} \AA$. FB 101 has a line of $100 \mathrm{~m} \AA$ at $\theta=0.19$, which is definitely weak. We can only conjecture that no sdB or $\mathrm{HBB}$ star has enhanced carbon lines, and that the lines are either slightly weak $(\sim 25$ percent) or weak in those stars in which it might be expected. (A few normal high-latitude stars had strong C II lines.) The case is unclear, however, since normal stars show steep changes in $\mathrm{C}$ II with type. The sdO stars are too hot for this criterion.

Turning to $\mathrm{Mg} \Pi, \lambda 4481$, however, a line which increases from $100 \mathrm{~m} \AA$ at $\theta=0.12$ to $350 \mathrm{~m} \AA$ by $\theta=$ 0.30 , we find that many of our stars have this line weakened. In the sdB stars FB 9, FB 103, and FB 178 have only about half the main-sequence $W(4481)$ at $\theta=0.20$. The upper limits given for the other sdB stars in table A3 approach the limit of what is expected, and no sdB star has an excessively strong $\mathbf{M g} \Pi$ line. The HBB stars, however, provide clear examples. FB 33, FB 73, FB 85, FB 134, and FB 189 have about normal $\mathrm{Mg}$ II, and some cool HBA stars $(\theta>0.50)$ were chosen because $\mathrm{Mg}$ II seemed normal, which permitted the Ca II line to be used to determine $\theta$. On the other hand, FB 107, FB 120, FB 137, and FB 144 have $W(4481)$ measured, but clearly about half or less the expected strength. Upper limits are significant for other stars, and suggest weakened $\lambda 4481$, on coudé or $90 \AA \mathrm{mm}^{-1}$ spectra, in FB 62, FB 91, FB 95, FB 123, FB 124, FB 
132 etc. Thus, in the HBB stars there may be a range of $\mathrm{Mg}$ abundance, but deficiencies occur quite frequently.

The surface helium abundance is fundamental to our classification of these EHB stars. The strength of $\lambda 4686$ separates our sdB from sdO stars; the weakness of the He I line in HBB stars separates them from the normal stars in the region where $\theta$ and $g$ are similar. Variations in $W(4471)$ are real in table $\mathrm{A} 2$, and become quite significant in table A3. Baschek et al. (1972) give $[\mathrm{He} / \mathrm{H}]$ as -1.0 in FB 9. Baschek and Norris (1970) give $[\mathrm{He} / \mathrm{H}]$ in $\mathrm{FB} 178$ as -1.2 . Clearly, we find both larger and smaller $W(4471)$ at nearly the same $\theta, g$. The dependence of He I strengths on temperature in normal stars is quite flat. We take the observed $W(4471)$, $300 \mathrm{~m} \AA-1200 \AA$ (in stars where it is observed) and to $<200 \mathrm{~m} \AA$ (where not observed, at $90 \AA \mathrm{mm}^{-1}$ ) as suggesting a range of $W(4471)$ of at least a factor of 6 . Note that the $W$ (4471) for FB 9 and FB 178, which have been analyzed at high dispersion, is near the top of the observed range for our sdB stars. Since the pressure broadening should not differ greatly over the limited range of $g$ for our stars, we may hazard an estimate of abundances. If $W(4471)$ varies as $N^{1 / 2}$, our range of line strength of six corresponds to a range of 1.6 in $[\mathrm{He} / \mathrm{H}]$, with some deficiencies less, but most greater, than in FB 9 and FB 178. The logarithmic deficiency of He in sdB stars, as compared to normal stars, is then roughly in the range -0.4 to -2.0 , at the surface.

Note, however, that the hot sdB stars do show some $\mathrm{He} \mathrm{I}$ lines, and that one we retain in table A3 as a sdB star, FB 103, could well have been listed as a sdO star in table A4. This star, Feige 66, described by Berger (1963) is not the bluest or hottest sdB star. But it has relatively strong $\mathrm{He} \mathrm{I}$ lines for its $\theta$, somewhat low surface gravity, and definitely anomalous composition. The strongest lines other than hydrogen and helium prove to be S III, an element not hitherto noted as enhanced in Population I peculiar B stars. FB 103 is hotter than most peculiar B stars, also. However, we do not see Si III lines on the two $9 \AA \mathrm{mm}^{-1} \mathrm{Pc}$ spectra we have, on which the $\mathrm{He}$ II was measured. The reason such behavior is worth noting is that FB 103 has small proper motion, and low surface gravity, i.e., an apparently high luminosity nearly approaching that of a main-sequence star. In fact, if classified as a normal star with a mass higher than the $0.66 \mathrm{~m}_{\odot}$ of EHB stars, its proper motion becomes almost unacceptably large, rather than small. In addition, S III is always much weaker than Si III in Population I spectra. Comparison of $\mathrm{Si}$ and $\mathrm{S}$ in a model at $\theta=0.183, \log g=4.0$, quite close to the parameters for FB 103, is instructive. Mihalas (1972a) gives $T\left(\mathrm{~m}=0.33 \mathrm{~g} \mathrm{~cm}^{-2}\right)=24,678^{\circ} \mathrm{K}$, and $n_{\theta}=4.23 \times 10^{14}$; this may be taken as a representative point equivalent to $\tau=0.1$. Evaluating the number of excited $\mathrm{S}^{++}$atoms, in a level at $18.17 \mathrm{eV}$, capable of producing the $\lambda 4253$ line, and comparing it with the number of excited $\mathrm{Si}^{++}$atoms in a level at 18.92 $\mathrm{eV}$, capable of producing the $\lambda 4552$ line, we find that 1.6 as many $\mathrm{S}$ as $\mathrm{Si}$ atoms are in relevant states. In $\mathrm{B} 0$ to B3 normal stars Si III is about three times as strong as the S III, both because the abundance of Si exceeds that of $S$, and because the $S$ III lines have smaller transition probabilities. In the sdO stars, Peterson gives a factor of 2 in favor of Si III. Thus, over a wide range of types and temperatures the $\mathrm{Si}$ III should be stronger, while in FB 103 it is $80 \mathrm{~m} \AA$ and $W(4253)$ is $140 \mathrm{~m} \AA$. We believe the surface abundance ratio $\mathrm{S} / \mathrm{H}$ is definitely anomalous in a star which otherwise has only weak lines of other elements. Berger (1963) noted the presence of S III on one of our early plates.

Another anomalous surface composition is that of the HBB star, FB 137, Feige 86. Tracing of our $9 \AA \mathrm{mm}^{-1}$ spectrum shows that the $\mathrm{S}$ III lines are absent, He I much weaker, Mg II much stronger. Sargent and Searle (1967) report the presence of Si II, Si III, P II, Fe II, and $\mathrm{Fe}$ III in FB 137, and conjecture that it is a halo star of peculiar composition, with weakened surface helium, such as is often seen in Bp or magnetic stars of Population I. We have intercompared the $9 \AA \mathrm{mm}^{-1}$ tracings of the two stars and find that Mg II, Si II, Si III, P II, and $\mathrm{Fe}$ II are weak or not present in FB 103, and that $\mathrm{S}$ III is not present in FB 137. Thus both these stars have peculiar compositions, but different ones.

FB 103 may have variations in line strengths and we are continuing to study it. The star is sufficiently faint that only two Pc spectra exist, one of which took two nights under adverse conditions. These plates have identical velocity, however. The $\mathrm{He}$ I lines are strange: on Pc $12153 W(4471)=960 \mathrm{~m} \AA$ as compared with $1180 \mathrm{~mA}$ on Pc 11680 , but $\lambda 4388$ is weak. $W(4686)$ is measured 330 and $340 \mathrm{~m} \AA$ on the two plates. The mean $W(4471)$ on two Pd plates was $870 \mathrm{~m} \AA$ and $W(4388)$, $150 \mathrm{mÅ}$. Sargent and Searle (1968), from a Lick spectrogram, give $W(4471)=1110 \mathrm{~m} \AA, W(4388)=$ $180 \mathrm{~mA} ;$ our mean values agree roughly with theirs. In table A3 we give the mean strengths of $\mathrm{He} \mathrm{I}$ from all four plates, $W(4686)$ from the two Pc plates, but use $D(0.2)$ only from the highest dispersion. On Pc 12153 $D(0.2)=11.6 \AA$, on Pc 11680, $13.4 \AA$; Sargent and Searle (1968) give $13.1 \AA$, in agreement with our stronger measures. In figure 12 we show the range of $M_{V}$ arising from such apparent changes. The fact that the He II line was not noted by several observers and could have been detected at any dispersion higher than $75 \AA \mathrm{mm}^{-1}$ reinforces the possibility of at least sporadic spectrum variability.

The importance of FB 103 is that it may provide a clue to the breakdown of whatever phenomenon is involved in the apparent helium deficiency of the HBB and sdB stars. If a stratification in composition becomes unstable, helium lines could strengthen or even be variable. Why sulfur is the favored heavy element, is, of course, less clear; selective radiation pressure may be involved (Michaud 1970). We find it best to retain FB 103 as a sdB star though it technically falls within the definition of a sdO star. Recent scans suggest that it has higher $T_{\text {eff }}$ and therefore higher gravity. It has the weakest He II line we have detected, its surface gravity is somewhat low, and it may provide a type of clue which eventually will explain the reasons for the weakness of helium on the horizontal branch, and its sudden strength in the sdO stars. We will see $(\S \mathrm{X})$ that, 
in fact, the sdO stars pose a question on the rarity of stars with $\mathrm{He}$ II lines weak, but still present. As a speculative scenario one might propose that helium (and the metals) settle out of the upper layers of $\mathrm{HB}$ stars. Instabilities, possibly caused by helium burning, drive convection and therefore mixing; the hydrogen envelope has low mass, the spectroscopically observable layers negligible mass. The extreme left end of the EHB is occupied only when the hydrogen envelope mass is negligible. Some of the HB stars with peculiar compositions, and relatively higher $\mathrm{He}$ than other $\mathrm{HB}$ stars, may exist because of such recent mixing events. It is interesting to recall the sdO stars in NGC 6397 (Searle and Rodgers 1966), a quite metal-poor cluster. From their best estimate of the modulus Searle and Rodgers find $M_{V} \simeq+1$. With our method and their data, $M_{V} \simeq$ -0.5 , probably too high since the $\mathrm{He}$ II line is strong. In any case this $\mathrm{sdO}$ is brighter than most of ours, and is above the EHB. It has $W(4686)=1.7 \AA$ and strong 14542. Strom and Strom (1970) studied VZ 1128 in M3 (a moderately metal-rich) cluster; this has $\theta=0.16$, $M_{V} \simeq-0.5$, and $W(4686)=1.2 \AA$. It is definitely brighter than the EHB stars, and, in fact, than most of our sdO. Clearly these are on different evolutionary tracks than the body of cluster stars.

Sandage's (1969) photometry of two sdB stars in M13 gives for S-A19 (17.06:, $-0.30,-1.09$ ) and S-A360 $(17.17,-0.21,-0.71)$. This shows that quite blue stars with color temperatures in the range $Q=-0.87(\mathrm{sdB})$ and $Q=-0.56$ (hot $\mathrm{HBB}$ or sdB stars) exist at $M_{V}$ near +3 . Sargent and Searle (1971, and unpublished) have spectra of both of these, and find a typical sdB spectrum and no trace of helium lines. They are kind enough to inform me that S-A19 has $T_{\text {eff }}$ near $33,000^{\circ} \mathrm{K}$. Thus M13 has stars like our hotter HBB stars, and typical sdB stars up to $33,000^{\circ}$, while, quite rarely, other clusters, NGC 6397 and M3 have stars above this EHB. If we estimate that about 500 horizontal-branch blue stars exist in the better studied clusters, and that only a few, much more easily found blue stars are known above the EHB, we may estimate that the brighter stage is only 1 percent or less of the total EHB life.

c) The Structure of the Extended Horizontal Branch

In $\S \mathrm{X}$ we will present results on the sdO stars and show that at least some lie near the high-temperature

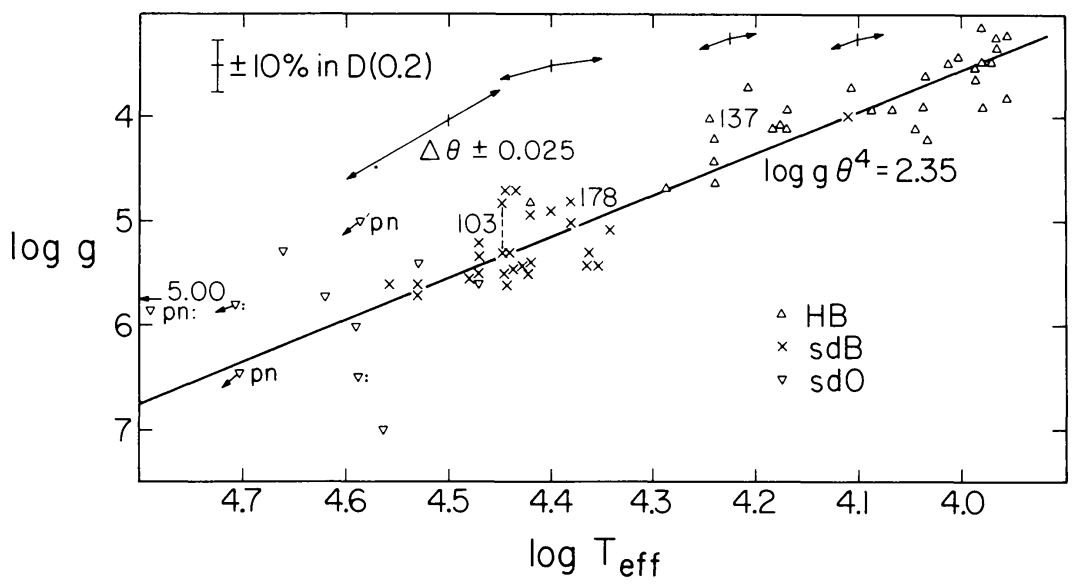

FIG. 12a.-The more reliable determinations of $\theta, g$ for the extended-horizontal-branch stars, $\mathrm{HB}, \mathrm{sdB}, \mathrm{sdO}$, and the log $g \theta^{4}$ line are shown. The error bar shows the effect of \pm 10 percent change in line profile $D(0.2)$. At several temperatures, arrows show the effect of temperature errors of \pm 0.025 . The symbol pn indicates a sdO nucleus of a planetary nebula.

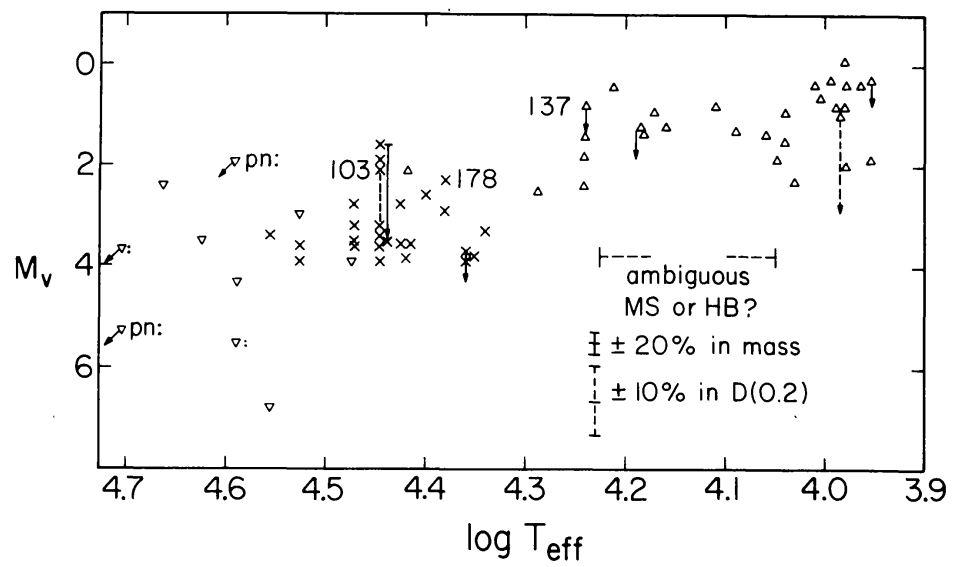

FIG. 12b.-The $\left(M_{V}, T_{\text {eff }}\right)$-relation for the same EHB stars as in fig. 12a. Error bars show the effects of changes of \pm 20 percent in mass and \pm 10 percent in $D(0.2)$. The region where the HB star crosses the main-sequence in the $(\theta, \log g)$-plane is indicated as "ambiguous." 
extension of the HB and sdB groups, in both the $(\theta, g)$ and the $\left(\theta, M_{V}\right)$-planes. For equal masses this suggests nearly constant $M_{b}$. In figure 12 we present diagrams showing those stars which we believe have at least moderately reliable data. The ( $\log T, g$ )-plane (fig. $12 a)$ presents the results of our measurements relatively directly. The $\left(\log T, M_{V}\right)$-plane involves both the assumption of equal mass, $0.66 \mathrm{~m}_{\odot}$, and the bolometric corrections. Of course, theoretical models for the interior structure are needed to explain the luminosity of the star as a function of the mass of the helium core and the hydrogen envelope. The approximate fit, in figure $12 a$, of the stars to the line of slope $4, \log g \theta^{4}=2.35$, means only that the stars have about the same mass-to-light ratio. Figure $12 a$ maps into figure $12 b$, on the assumption of constant mass (and therefore light); a $(\log T$, $M_{b}$ )-diagram would resemble figure $12 b$ except for having a nearly horizontal slope. In figure $12 a$ we show some of the effects of errors in $D(0.2)$ and in $\theta$, derived from table 3. Changes in $D(0.2)$ have the same effect as changes in mass, vertical displacement in $\log g$, and produce 2.5 times as large a displacement in the $M_{V}$ diagram. For $\log T_{e}<4.55$ almost all stars lie within a band \pm 0.4 in $\log g$. About half of this scatter could be caused by \pm 10 percent errors in $D(0.2)$. In figure $12 b$ we show the rather small effects of 20 percent changes in mass, presumably near the upper limit of plausibility for halo stars.

The unfortunate effect of errors in temperature adopted is not so much in the consequent errors in the derived $\log g$ as in the blurring of any natural boundaries between different types of stars. If there were a real evolutionary discontinuity at the hot end of the HBB stars found in globular clusters, near $\log T_{\text {eff }}=4.3$, it would be blurred. Real gaps or turning points in evolution would not be seen if our errors of $\theta$ exceed \pm 0.025 . However, the sdB star in M13 suggests that there is no gross break in that cluster's EHB. The change in bolometric correction for $\Delta \theta= \pm 0.023$ is about \pm 0.40 mag at $\theta=0.15$ and $\pm 0.15 \mathrm{mag}$ at $\theta=0.35$, introducing an extra, small spread in $M_{V}$ (fig. 12b). Thus, much of the spread found in these diagrams can be understood as arising from errors in observed quantities. Furthermore, it still may be optimistic to hope that model atmospheres can give $\log g$ without a systematic error as large as 0.3 , which results in a vertical shift of 0.75 mag. We have, further, an extra source of error near the intersection of the main sequence and the horizontal branch, in the range $\theta=0.30-0.40$. Ambiguity in selecting a star as normal or HB could result in inclusion in these figures of objects with too small a $g$, at $\theta \lesssim$ 0.35 , and with too large a $g$ at $\theta \gtrsim 0.35$ (see fig. 4).

Evolutionary explanations of a "sequence" with nearly constant mass-to-light ratio are difficult for $\log T>$ 4.30. We do not wish to overemphasize continuity, but merely present the evidence to stimulate further extension of theoretical models beyond the present work of Faulkner (1966, 1972), Rood (1973), and Demarque (see, for example, Demarque and Mengel 1971, 1972, etc.). In an approximate way we can visualize that most of these stars have a thin hydrogen envelope and a helium core; this structure, like that of a red giant, gives nearly constant luminosity, but a radius dependent on many details of evolutionary age and envelope-tocore mass. The high temperature limit is set by an intersection with the pure helium main sequence (L'Ecuyer 1966; Biermann and Kippenhahn 1971). The ZAMS for a pure helium star of $0.5 \mathrm{~m}_{\odot}$ has $\log$ $T_{\text {eff }} \simeq 4.6$, not far from the sdO region. The light-tomass ratio in solar units is $\log \left(L / L_{\odot}\right)\left(\mathfrak{m} / \mathfrak{m}_{\odot}\right)^{-1}=$ +1.23 for $0.4 \mathrm{~m}_{\odot}, 1.60$ for $0.5 \mathrm{~m}_{\odot}$, and 2.05 for $0.7 \mathrm{~m}_{\odot}$. L'Ecuyer's evolutionary tracks run upward to the left, however, and in $10^{7}-10^{8}$ years reach the region of the hotter planetaries. In table 2 we gave an assortment of possible values of the constant $\left\langle\log g \theta^{4}\right\rangle$, from 2.19 to 2.38. Dependent on the mean luminosity, which we obtained from fitting the EHB to the globular clusters, as $M_{b}=+0.5$, we place $\log \left(L / L_{\odot}\right)\left(\mathfrak{m} / \mathfrak{m}_{\odot}\right)^{-1}$ in the range 1.99 to 1.80 . Thus, if helium-core stars are displaced at constant luminosity, by a hydrogen envelope, toward lower temperature from the helium main sequence, the mass should be $0.6-0.7 \mathrm{~m}_{\odot}$.

\section{THE SUBDWARF O STARS}

a) General Considerations

The very hot subdwarfs have important evolutionary status, representing one or more evolutionary tracks. Only some half-dozen have been studied spectroscopically, by Peterson (1969) and Tomley (1970). Some, the nuclei of planetary nebulae, are highly luminous and very hot; others have low luminosity, like $+28^{\circ} 4211$ (Greenstein 1952) and do not now have surrounding nebulosity. Planetary nuclei may have emission lines, and be unstable, even the nuclei of the relatively lowluminosity Abell planetaries (Greenstein and Minkowski 1964). However, the lines of the nebula often prevent analysis of the absorption lines of the stars. Most isolated sdOs we study do not have this problem, although the Abell nuclei have very weak Balmer lines.

Table A4 contains data for $30 \mathrm{sdOs}$, the largest number yet listed. They are defined by Balmer lines strong for their color, and by the presence of strong $\mathrm{He}$ II, $\lambda 4686$. For many the data presented must be incomplete; the helium lines are relatively easily measured, with errors better than 20 percent, but the hydrogen lines are very shallow $\left(R_{c}\right.$ from 0.05 to 0.40 , averaging 0.20 ), and the Pickering series of $\mathrm{He}$ II is superposed. Thus, the $\lambda \lambda 4101,4340$ profiles are composites of $\mathrm{H}$ and $\mathrm{He}$ II, especially when $\lambda 4686$ is very strong. We mark by asterisks or colons in table A4 the results, which we believe unreliable, for FB 3, FB 26, FB 31, FB 56, FB 57, FB 60, FB 105, FB 106, FB 138, FB 161, FB 166, and FB 179 which suffer most from He II contamination; for these, both $\theta$ and $g$ (from hydrogen) are affected by He II. For most, $D(0.2)$ is not measurable but $D(0.1)$ could be used. These stars are identified in figures $4,5,7,8$ by a circle surrounding the triangular sdO symbol. The more reliable stars have $\left\langle M_{V}\right\rangle=+4.3 \pm 0.5$, with $\sigma\left(M_{V}\right)= \pm 1.7$; the unreliable stars have $\left\langle M_{V}\right\rangle=+2.8 \pm 0.8$, with $\sigma\left(M_{V}\right)=$ \pm 3.0 . Either the $\mathrm{He} / \mathrm{H}$ ratio is larger, or the surfacegravity dependence of the line broadening is different 
in the two groups. We will see that the behavior of He I and $\mathrm{He} \mathrm{II}$ is confusing.

The existence of the planetary nebula K648 in, and with the same radial velocity as the globular cluster M15, provides a direct measure of the luminosity of a halo sdO star. O'Dell, Peimbert, and Kinman (1964) find that its central star has $m_{v}=14.37$, which leads to $M_{V} \simeq-1.6$, far above the horizontal branch of the cluster. In table A4 only FB 56 is so bright, and we will show that it is, in fact, fainter than our spectroscopic method indicates. The planetary K648 has apparently normal $\mathrm{He} / \mathrm{H}$, and a low $\mathrm{O} / \mathrm{H}$ abundance. Its electron temperature is high; since the central star may be very blue, it is likely that it is hot. Unfortunately, no direct estimates of its characteristics can be made. Its bolometric magnitude, however, would be far greater than that of the average sdO star we discuss here. Apparently the central star of K648 represents a quite different, and probably earlier, stage of evolution than do the sdO stars near the end of the EHB. This is supported by the O'Dell et al. estimate of $0.2 \mathrm{~m}_{\odot}$ for the planetary nebulae. O'Dell (1968) gives $\theta=0.167$ and $M_{b}=-4.1$ for the star, resulting in a radius $2 R_{\odot}$. Most of our sdO stars and the fainter planetary nuclei have $R \simeq$ $0.1 R_{\odot}$.

There are important sources of systematic error in our work on the sdOs, and we offer these results as a first exploration of an almost completely unexplored region of the H-R diagram. In $\S \mathrm{VI} a$ we discuss difficulties in the temperature scale for the white dwarfs which result from the effect of Balmer lines on the $U B V$ colors; to a lesser extent, since the Balmer lines are much weaker, this same effect must persist in the sdOs. The helium-line-blanketing corrections to model atmospheres must be serious as log $g$ becomes large, and the farultraviolet lines strengthen. Thus the $(\theta, Q)$-calibration is uncertain, and interpretation difficult. Finally, inspection of figure 3 shows that we have had to interpolate very roughly, for $\theta<0.17$, between Shipman's whitedwarf models and the few Mihalas non-LTE high- $T$, high log $g$ models.

\section{b) Helium Line Strengths and sdO Temperatures}

The roughness of our exploration must be emphasized because of the outstandingly peculiar nature of these stars. This interest is justified in part by the great strength of $\mathrm{He}$ II and He I lines shown in figure 13. The sdO stars are defined by easily measurable $\lambda 4686$. However, the apparently $y^{5}$ coolest, FB 105, with $\theta=$ 0.23 has $W(4686)=2.0 \AA$; from this to the hottest, at $\theta=0.10, W(4686)$ changes only slightly, to about $2.4 \AA$ in the high- $g$ stars, and to $1.5 \AA$ in the low- $g$ stars (the difference presumably arising from pressure broadening).

\footnotetext{
${ }^{5}$ FB 105 has a surprisingly low color temperature. Consequently, the $\log g$ derived is the lowest we find among the sdO stars, placing it, in fact, above the sdB or even normal stars. This is quite inconsistent with the small number of Balmer lines and the strong $\mathrm{He}$ II and $\mathrm{He}$ I lines. We believe that the star must be bluer than the values $B-V=-0.25, U-B=-0.88$ we used, since a tentative value $V-R=-0.54$ (from Margaret Penston) is somewhat unexpected in combination with these. Possibly the star is composite.
}

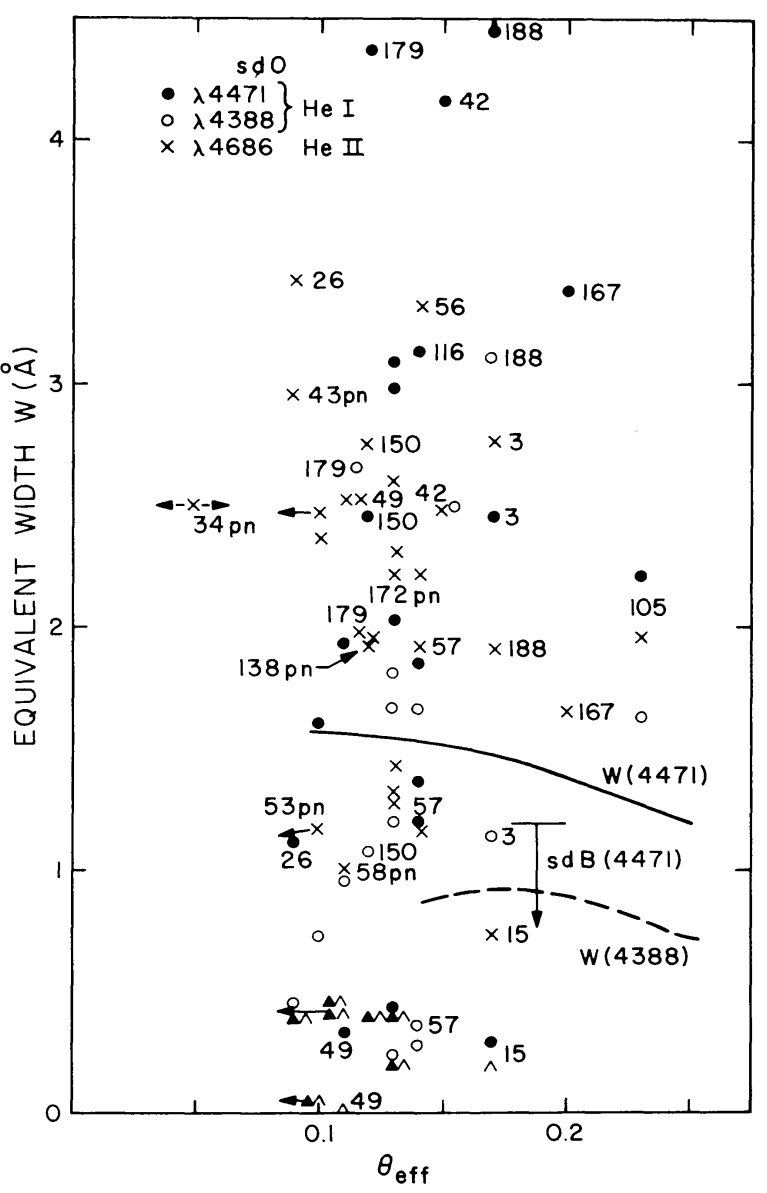

FIG. 13.-The He II strengths, $W(4686)$ in sdO stars are shown with crosses. The mean $\mathrm{He} I$ line strengths in normal stars are shown. Filled circles, sdO W(4471); open circles, $W(4388)$; Carets, upper limits for He I, $\lambda 4388$; filled triangles, for $\lambda 4471$. The line and arrow indicate the strongest $\lambda 4471$ of $\mathrm{He} \mathrm{I}$ in sdB stars, much weaker than in sdO stars.

There is a general decrease in He I strength with decreasing $\theta$. But individual stars, grouped at their nominal temperatures, show large variation in $R^{\prime}=W(4471)$ / $W(4686)$, the $\mathrm{He} \mathrm{I} / \mathrm{He}$ II ratio, as well as in the absolute strengths. For example, FB 3 has $R^{\prime}=0.89$, FB 188 has $R^{\prime}=2.77$, both at $\theta=0.17$. A radical difference in the surface abundance of helium (and, of course, of $R^{\prime}$ ) is found between the sdO and the hot sdB stars. No matter how we reshuffle the temperatures within the sdO group we doubt that it will be possible to explain their helium spectra with identical surface composition. The two groups overlap in temperature and apparently do not differ substantially in surface gravity. The observed differences in helium-line strength are not dependent on the uncertainties listed above, which affect the hydrogen lines and $\log g$ derived from them.

In addition to the lines measured, inspection of the spectra shows $\lambda \lambda 4200,4542$ of the Pickering series in most stars; FB 15, FB 77, and FB 106 with weak $\lambda 4686$ do not show them. A strong, possibly interstellar $\lambda 3933$ line is noted in FB 3, FB 166 and a feature at $\lambda 3933$ is measured in. others. However, at low dispersion, and 
with strong helium, there are blending lines of $\mathrm{He}$ I which would be unresolved from $\mathrm{Ca}$ II. Some of the Abell planetary nuclei in table A4 are reddened and probably have interstellar K-lines. Data for line intensities of some stars was taken from Peterson (1969); details of other lines can be found there. We measured our spectra of FB 131 and quote her $W$ for FB 49, FB 150 , and FB 179, but have reevaluated $\theta, g, M_{b}, M_{V}$ for all these stars by our methods. We quote our own data for FB 29 (Greenstein, Sargent, and Haug 1970), FB 34 (Greenstein 1972). Other lines characteristic of intrinsically bright $\mathrm{O}$ stars are seen in a few of these stars and were used in velocity measurement. However, Greenstein and Eggen (1966) already noted a strange feature of the sdO stars. FB $49\left(+75^{\circ} 325\right)$ and FB 131 (HZ 44) have essentially identical temperatures $(\theta=$ 0.11 and 0.13$)$, the same $W(4686)$ and $\log g=5.3 ; \mathrm{FB}$ 49 shows almost no lines except $\mathrm{He}$ II and weak He I; FB 131 has about a hundred lines of ions of the CNO group, and strong $\mathrm{He}$ I. In fact, Greenstein and Eggen (1966) divided the few sdOs then seen at reasonable dispersion into a rich-line and weak-line group. Too few of the sdOs in table A4 are sufficiently bright for us to have explored further this phenomenon, connected either with heavy-element abundances, or with mixing processes.

The helium spectrum shows no significant dependence of the means of $W(4686), W(4471), W(4388)$ on $\theta$ for the $10 \mathrm{sdO}$ stars with $\log g<5.5$. For $\log g \geq 5.5$, the 12 sdO stars show $\mathrm{He}$ II $W(4686)$ decreasing (at a marginal level of significance) with decreasing temperature; however, He I, $W(4471)$ and $W(4388)$, do clearly increase with decreasing temperature. Thus, changes in $\mathrm{He}^{+}$recombination with $\theta$ appear in the $\mathrm{He}^{+} / \mathrm{He}$ ratio at high gravity, but less clearly so at low gravity. On the average, $\mathrm{He} \mathrm{I}$ lines are stronger in low- $g$ sdO stars, but $\mathrm{He}$ II is about the same. The He I lines in almost all sdO stars are stronger than in normal stars. (We show in fig. 13 the mean strengths of triplets and singlets, taken from the normal and sdB stars of fig. 10.) But in sdO stars with $\theta<0.15$, there is a very large spread in observed He I lines, with many having less than normal $W(4471)$. There is also a spread in $W(4471)$ in the hotter sdB stars; some have no line stronger than $200 \mathrm{~m} \AA$ visible; at $\theta=0.17$ those that show He I have a mean $W(4471)$ of $680 \mathrm{~m} \AA$. The eight sdO stars at $\theta=0.16$ have mean $W(4471)=1670 \mathrm{~m} \AA$. The mean surface gravities are close, $\log g=5.9$, for the sdB stars, $\log g=5.1$ for the sdO stars. Helium is mostly singly ionized at $\theta=0.16$ in the Mihalas (1972a) models, at $\log g=4.5$ and at $\tau \simeq 0.1$.

If we assume that, independent of helium abundance, the ratio $R^{\prime}=W(4471) / W(4686)$ must be a temperature indicator, we can order the sdOs into temperature groups by values of $R^{\prime}$. To obtain about equal numbers in each group we select as the hottest sdO those with $R^{\prime}<0.40$, intermediate $0.40 \leq R^{\prime} \leq 0.85$, cool $R^{\prime}>$ 0.85 . The mean $\theta^{\prime}$ 's (based on color temperature) are then $0.11,0.14,0.16$, i.e., in the correct order; the coolest group has also the largest mean $\log g$. The sdB stars observed at coudé or $90 \AA \mathrm{mm}^{-1}$ dispersions have
$R^{\prime}>10$ and nominal $\theta$ in the range $0.15<\theta<0.22$. The change in the $\mathrm{He} / \mathrm{He}^{+}$ratio may seem large for so small a temperature change. Given the low helium concentration in sdB stars it is difficult to estimate the true temperature difference between $\mathrm{sdO}$ and $\mathrm{sdB}$ stars by this means. Note, however, that the $\mathrm{He}$ II lines originate from levels approximately $52 \mathrm{eV}$ above the levels producing $\mathrm{He}$ I lines, so that the logarithmic population ratios would vary steeply. To clarify this we have non-LTE model atmospheres by Mihalas (1972a). He gives the structure for $\log g=4.5$ in the range from $55,000^{\circ}$ to $25,000^{\circ} \mathrm{K}$ effective temperature, and includes the number density of ground-state neutral and singly ionized helium atoms. Although the gravity is too low for our purposes, the tables provide the largest published body of data. The independent variable is $\mathrm{m}$, the mass in grams $\mathrm{cm}^{-2}$ above a point, and the temperature, $T(\mathrm{~m})$, is listed. Since electron scattering is the dominant opacity we chose representative points near $0.3 \mathrm{~g} \mathrm{~cm}^{-2}$; these vary slightly from model to model. They correspond roughly to $\tau=0.1$ in the continuum. At $27,500^{\circ} \mathrm{K}$ only a model at $\log g=4.0$ was available. The observable neutral helium spectrum was represented by a level at $21 \mathrm{eV}$, that of ionized helium by one at $48 \mathrm{eV}$. At a mass of $0.3 \mathrm{~g} \mathrm{~cm}^{-2}$ the local temperature should represent the excitation fairly well. In figure 14 we show the populations for $\mathrm{He}$ I, $\lambda 4471$ and $\mathrm{He}$ II, $\lambda 4686$ so derived, together with the ratio $R=\log$ $N(\lambda 4471) / N(\lambda 4686)$. Trials at smaller mass depths gave similar results. Note that $R$ changes by 6 from $\theta=0.09$ to 0.18 . The population of the He I excited level drops steadily at $\theta<0.17$, and that of the He II excited level rises rapidly, and has a flat maximum near $\theta=0.12$. At higher $g$ these phenomena would occur at slightly higher temperature. (We would expect $P_{e} \propto g^{1 / 2}$; the ionization and broadening change in opposite directions.) If both lines are collisionally broadened by ions equally, $\log R^{\prime}=\log W(\lambda 4471) / W(\lambda 4686)$ would

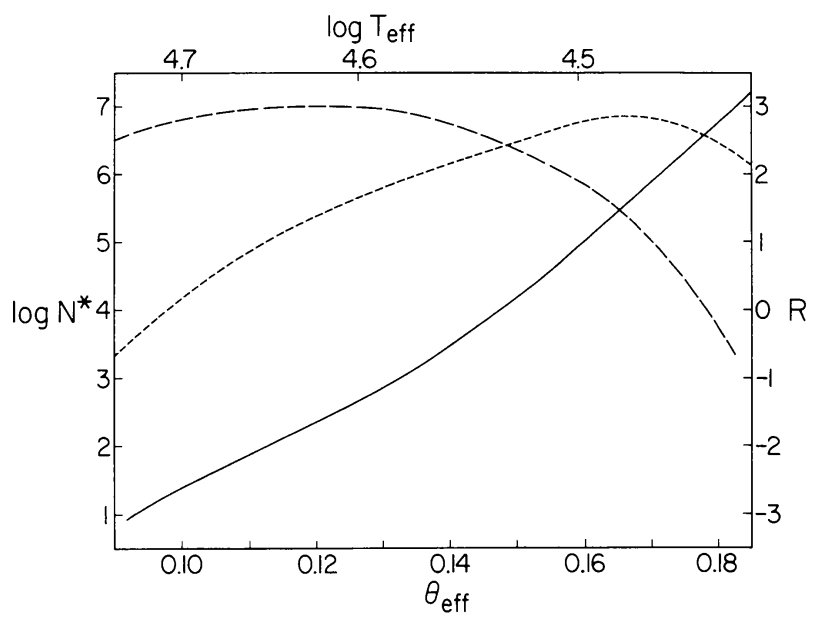

Fig. 14.-Theoretical populations of $\mathrm{He} \mathrm{I}$ and $\mathrm{He}$ Ir levels producing $\lambda 4471$ (short-dash curve) and $\lambda 4686$ (long dashes), from Mihalas non-LTE models at $\log g=4.5$; the model at $\theta=0.183$ has $\log g=4.0$. The solid curve gives, $R$, the logarithm of the ratio of populations. 
vary as $R / 2$, so that we expect a range of 1000 in the ratio of equivalent widths. This is well beyond our capabilities to observe. The He I lines does not weaken substantially, in absolute concentration until $\theta<0.12$, although hotter stars tend to produce weaker lines for equal numbers of atoms.

Stars with no visible $\mathrm{He}$ I lines probably have $\theta<$ 0.11 . In table A4 the nuclei of planetary nebulae (FB 43 , FB 53, FB 58, FB 138, FB 172) all lack detectable $\lambda 4471$, i.e., $T_{\text {eff }}>45,000^{\circ} \mathrm{K}$. Only two of the hottest stars, FB 64 and FB 106, also lack $\lambda 4471$. The accurate $U B V$ photometry of $+28^{\circ} 4211$ gives a highly reliable $Q=-1.02$; from figure $1, \theta=0.09$ making this one of the hottest sdO stars. It is spectroscopically indistinguishable from the nucleus of a planetary nebula and the He r line is absent at $18 \AA^{-1} \mathrm{~mm}^{-1}$ even with $W(4686)=$ $2.4 \AA$ (Greenstein 1956) and the Pickering series visible. A detailed study of the $\mathrm{He} \mathrm{I} / \mathrm{He}$ II behavior in the hottest sdO star and in more typical planetary nuclei might permit us to establish whether there is a gap in temperature between the two classes of stars. Existence of such a gap could clarify the downward evolutionary tracks, or establish any genuine differences in their origins. The stars could be placed in order of temperature to at least $45,000^{\circ} \mathrm{K}$ by the $\mathrm{He} \mathrm{I} / \mathrm{He}$ II line ratio and then the color-temperature scale could be recalibrated. This would improve on a colorimetric scale not yet sufficiently calibrated and definitely best studied by far-ultraviolet observation from a satellite. Provisionally, we suggest that FB stars with $R^{\prime}<0.30$ are among the hottest known stars with absorption-line spectra; the list of candidates for $T \geqslant 50,000^{\circ} \mathrm{K}$ include the Abell planetary nuclei above mentioned, plus FB 49, FB 64, FB 106, and FB 186.

\section{c) Kinematics and the Luminosities}

The sdO star list in table A4 included three very blue Lowell GD stars discovered because of their proper motion. The rest of the list were selected by blue color, so that the statistics of sdO star motions are essentially those of a group selected by apparent magnitude. Their luminosity per volume of space should be several magnitudes fainter. There are six stars with sufficiently large motions to justify computation of $U, V, W$ motions, with a goal of setting upper limits to their luminosities. Radial velocities were available for most of the stars with reliable proper motion. They may, conceivably, be affected by helium pressure shifts, which tend to be negative. Stars with absolute proper motions $>0$ ". 015 and reasonably small quoted errors were treated by the method of reduced proper motion. Their mean motion was 0".050 (11 stars); the mean $\left\langle m_{V}+5 \log \mu\right\rangle=5.39 \pm 0.43$. The corrected dispersion in radial velocity was $\pm 58 \mathrm{~km} \mathrm{~s}^{-1}$; as shown in the Appendix, the mean tangential velocity so derived yields a mean absolute visual magnitude $\left\langle M_{V}\right\rangle=$ $+2.9 \pm 0.4$ (m.e.) and $\sigma\left(M_{V}\right)= \pm 1.4$. The direct mean of the spectroscopically determined $M_{V}=$ $+4.0 \pm 0.6$, with $\sigma\left(M_{V}\right)= \pm 1.8$, for these same stars. Had we used all sdO stars including some with very small proper motions, $\left\langle M_{V}\right\rangle=+2.3 \pm 0.6, \sigma\left(M_{V}\right)=$ \pm 2.4 , omitting Abell stars. One ninth-magnitude sdO star, FB 49, has a negligible motion, 0.012, and is possibly intrinsically brighter.

The luminosity from kinematics is not in as satisfactory agreement with the spectroscopic value as for the $\mathrm{HB}$ and sdB stars. Nevertheless, these sdO stars belong to the old disk and halo population, given the high tangential velocity. The fit of the sdO stars to the high temperature end of the $\mathrm{sdB}$ stars, i.e., a further prolongation of the EHB, however, is probably not an accident. If we adopt $M_{b}=+0.5$ for the cool horizontalbranch stars, the above derived $M_{V}$ determines the mean bolometric correction of $3.2 \mathrm{mag}$, i.e., $T=$ $34,000^{\circ} \mathrm{K}$.

But satisfying as this may be, it is clear from the few stars of known larger proper motion that the luminosities are not all correct. FB 56 (a Lowell star, GD 298) is ascribed a spectroscopic luminosity of -1.4 . The resultant distance of $2.7 \mathrm{kpc}$ is quite incompatible with any detectable proper motion. As table 7 shows, its $U$, $V$ exceed $1000 \mathrm{~km} \mathrm{~s}^{-1}$. However, FB 56, FB 57, and FB 60, all Lowell GD stars, are noted as probably having unreliable $\theta, g, M_{V}$ because of the strength of the He II lines. We list these together in table 7, which gives the $U, V, W$ for those stars for which we had relatively reliable proper motions and radial velocities. The unacceptably large velocities result from the large distances derived using the $M_{V}$ from table A4. We can, however, assume that these stars do not have space motions with any component greater than $200 \mathrm{~km} \mathrm{~s}^{-1}$, and can reduce the distances to values such that this hypothesis is true. In particular, it is interesting that all $V$-components are negative (i.e., retrograde orbits)

TABLE 7

Adjustment of Space Motions of sdO Stars

\begin{tabular}{|c|c|c|c|c|c|c|c|c|}
\hline \multirow[b]{2}{*}{$\mathrm{FB}$} & \multirow{2}{*}{$\frac{M_{V}}{\text { table A4 }}$} & \multirow[b]{2}{*}{$\begin{array}{c}U \\
\left(\mathrm{~km} \mathrm{~s}^{-1}\right)\end{array}$} & \multirow[b]{2}{*}{$\begin{array}{c}V \\
\left(\mathrm{~km} \mathrm{~s}^{-1}\right)\end{array}$} & \multirow[b]{2}{*}{$\begin{array}{c}W \\
\left(\mathrm{~km} \mathrm{~s}^{-1}\right)\end{array}$} & \multicolumn{4}{|c|}{ REVISEd LUMINOSITIES } \\
\hline & & & & & $M_{V}^{\prime}$ & $\begin{array}{c}U^{\prime} \\
\left(\mathrm{km} \mathrm{s}^{-1}\right)\end{array}$ & $\begin{array}{c}V^{\prime} \\
\left(\mathrm{km} \mathrm{s}^{-1}\right)\end{array}$ & $\begin{array}{c}W^{\prime} \\
\left(\mathrm{km} \mathrm{s}^{-1}\right)\end{array}$ \\
\hline $\begin{array}{r}56 \ldots \\
57 \ldots \\
60 \ldots \\
77 \ldots \\
106 \ldots \\
131 \ldots\end{array}$ & $\begin{array}{l}-1.4 \\
+1.6 \\
+0.1 \\
+5.6 \\
+1.0 \\
+2.5\end{array}$ & $\begin{array}{rr}-1148 \\
-\quad 63 \\
+\quad 523 \\
+119 \\
+\quad 105 \\
+\quad 90\end{array}$ & $\begin{array}{r}-1037 \\
-\quad 397 \\
-\quad 328 \\
+\quad 4 \\
-\quad 257 \\
-\quad 18\end{array}$ & $\begin{array}{r}+199 \\
+204 \\
-386 \\
+75 \\
-\quad 26 \\
-\quad 1\end{array}$ & $\begin{array}{l}+2.4 \\
+3.3 \\
+2.4 \\
+5.6 \\
+1.5 \\
+2.5\end{array}$ & $\begin{array}{l}-224 \\
-25 \\
+188 \\
+119 \\
+\quad 83 \\
+90\end{array}$ & $\begin{array}{l}-184 \\
-180 \\
-112 \\
+\quad 4 \\
-205 \\
-\quad 18\end{array}$ & $\begin{array}{r}+183 \\
+98 \\
-108 \\
+75 \\
-15 \\
-\quad 1\end{array}$ \\
\hline
\end{tabular}


before and after such adjustments. The second half of table 7 gives the new assumed $M_{V}{ }^{\prime}$, and the $U^{\prime}, V^{\prime}, W^{\prime}$ forced to be near or below $200 \mathrm{~km} \mathrm{~s}^{-1}$. The $M_{V}$ for stars with initially acceptable $U, V, W$ were not changed. The average $M_{V}^{\prime}-M_{V}$ is $+1.3 \mathrm{mag}$, corresponding to a change of +0.52 in $\log g$. We can easily accept such an adjustment as reasonable, given the sensitivity of $g$ to $\theta$ at high temperature. The change in temperature suggested by the ratio of $\mathrm{He} \mathrm{I} / \mathrm{He}$ II line strengths is compatible with such adjustment in $\log g$. We believe, however, that even with the necessary adjustment, the $U^{\prime}, V^{\prime}, W^{\prime}$ values now suggested prove that many of these sdO stars belong to the halo population rather than to Population I. If we had decided to reduce $U, V, W$ to below $20 \mathrm{~km} \mathrm{~s}^{-1}$, the distances for most stars in table 7 would be reduced by a factor of 10 , the luminosity by 100 , i.e., the $\left\langle M_{V}\right\rangle$ would be +8 , close to that of the hot white dwarfs. The sdO line spectra differ substantially from the spectra of the DO stars (hot white dwarfs); the line broadening would make $D(0.2)$ too large, but would be far too great to permit observation of $\mathrm{CNO}$ ions such as found in the apparently brighter sdO stars seen at high dispersion. We suggest that many sdOs are halo stars and others old disk (the planetary nuclei, for example), with comparable $\theta, g$.

\section{THE HOT WHITE DWARFS}

\section{a) Introduction}

The 27 white dwarfs here studied have data shown in table A5. Twenty-one of the 27 were found as very blue in colorimetric surveys; some are also known propermotion stars. These surveys (Feige, Humason-Zwicky, and Tonantzintla) revealed stars of such unusual $U B V$ colors that spectra were originally taken to ascertain whether they were white dwarfs, thus creating a selection effect favoring white dwarfs. This selection is somewhat balanced by the fact that they are among the apparently faintest stars observed. A few FB white dwarfs in table A5 were observed because they were very blue stars of small proper motion and would have been found if a colorimetric survey had covered their area. Most such have Lowell color class -1 ; FB 165 is also a Tonantzintla star (TON 816). The table therefore contains a large list of hot white dwarfs found and confirmed because of their blue color; the proper-motion selection in the Eggen-Greenstein lists favors the high-velocity stars. Twelve of the 27 are types DC, DO, DB, or DAwk, so that a relatively straightforward modification of our colorimetric method, discussed in $\S \mathrm{VI} a$, gave the temperatures. Because of the sensitivity of $Q$ to the Balmer lines, we restrict this list to stars with relatively weak (or no) hydrogen lines. The temperatures conform fairly well to a modification of Shipman's (1972) scale which is based on model atmospheres and multichannel spectrophotometry by Oke (1974). Nevertheless, a few DAwk stars seem still to be excessively hot, and with strong Balmer lines have been ascribed too high a surface gravity (e.g., FB 45, FB 94, and FB 165), while others seem to have too low gravity (e.g., FB 44,
FB 55, and FB 127). The last is almost certainly hydrogen-deficient. The helium stars, DB, in table A5 have He I lines stronger than any normal stars; the DO stars have $\mathrm{He}$ II stronger than sdO stars.

Plotted in figure 4 , the $\theta, \log g$ of these white dwarfs merge with those for the most extreme sdO stars, as do the He I and $\mathrm{He}$ II line strengths. The masses should be comparable, given the upper limit to white-dwarf masses. Therefore, in figures 7 and 8 , the luminositytemperature diagrams, they also merge with the sdO stars. Although the temperature scales are uncertain above $\log T=4.50$ any errors in $\log g$ should be similar for white dwarfs and sdO stars; this topological continuity should be retained, unless, which seems quite unlikely, there are no white dwarfs as hot as sdO stars. Therefore, the bolometric luminosities of the hottest white dwarfs are as indistinguishable from those of the high surface gravity sdO stars as are their colors, and many of their spectra. There are, of course, serious problems in linking sdO stars directly to white dwarfs on evolutionary tracks. Both occur in the young and old populations, both commonly have abnormal surface hydrogen-to-helium abundance ratios. However, some sdO stars are much hotter, e.g., nuclei of planetary nebulae with $\log T>5.0$, from the Zanstra method. In addition, some sdO stars seen at high dispersion have relatively sharp lines of many highly ionized elements, while no white dwarf has yet shown any sharp lines, nor any of the strong lines $\mathrm{C}$ III, $\mathrm{C}$ IV, $\mathrm{N}$ III, N IV, Si III, Si IV, O vI seen in sdO stars. It will be especially important to study from space the ultraviolet continua and line spectra at the transition from sdO stars to the hottest white dwarfs. From Oke (1974), or Greenstein's multichannel observations (noted as MC in the "quality" column of table A5), it is clear that the hot white dwarfs are very hot indeed, from comparison with model atmosphere fluxes. With $\log g$ derived from the hydrogen-line profiles five of the 27 stars are more luminous than the Sun; none of the over 50 stars in figures 4 and 5 of Eggen and Greenstein (1965) are as bright as the Sun. Hot white dwarfs are, in fact, quite rare in space; plotted in the $\left(T, M_{b}\right)$-plane the classical white dwarfs begin to appear and just overlap the lower right-hand corner of our figure 7. In table 4 of Eggen and Greenstein (1965) there are 16 DA white dwarfs listed as members of galactic clusters, the brightest of which is $M_{V}=+11$; the clusters (Pleiades, Hyades, Praesepe) have ages $3 \times 10^{7}<t<10^{9}$ year. The hot white dwarfs probably have shorter cooling times than the age of the Pleiades. One should imagine the lower right-hand corner of figures 7 and 8 filled in with at least 10 times more stars, to picture the relative frequency of normal DA's with $\log T<4.5$, correctly.

An important additional fact is that no objects exist between the $\mathrm{HBB}, \mathrm{sdB}$, and $\mathrm{sdO}$ stars and the white dwarfs. Note the clear absence in figure 4 of stars with $\theta \simeq 0.20$ and $5.6<\log g<7.2$; by $\theta>0.25$ there are no stars with $4.7<\log g<7.5$. In figures 7 and 8 there exists, therefore, a wedge-shaped empty area, below the $\mathrm{HBB}$ and sdO stars and above the white dwarfs, stretching over $8 \mathrm{mag}$. 


\section{b) Kinematics}

If we are to find the evolutionary stage just preceding the hot white dwarfs we need some knowledge of population types. We therefore present approximate space motions, $U, V, W$, shown in table 8 . The luminosities from table A5 were used; the radial velocities had to be assumed zero (which reduces $\sigma_{W}$ for stars in the galactic polar caps). When the spectra did not provide a new $M_{V}$ (e.g., in a DB star) values of $U, V, W$ given by Eggen and Greenstein (1965) were adopted. The DA Hyades members are listed as one star, FB 32; FB 70 and FB 127 have poor $M_{V}$; others have poor proper motions. If we have made errors in estimating the temperature as too high, we will overestimate the star's luminosity, and therefore give too large a space motion. We believe, however, that the motions in $W$, and the total motions, $T=\left(U^{2}+V^{2}+W^{2}\right)^{1 / 2}$, are sufficiently accurate to provide some valuable indications.

Too few motions were available from table A5 for good statistics. We therefore add to table 8 the $U, V$, $W$ for some of the hottest of the EG white dwarfs of types DO, DB, DAwk from Eggen and Greenstein (1965). These were limited also to stars found in the colorimetric surveys F, HZ, TON, LB (Luyten blue), and LDS (Luyten double-star). These blue EG stars all were in the single-valued $\left(M_{V}\right.$, color) range; therefore, we use the so-called upper sequence luminosity and space motion. A few stars are repeated as FB or EG stars, to show the quality of the agreement. Note a few discrepancies (FB 127, FB 145) whose luminosities and motions are increased over the values given by EG. Only $U-V$ colors are available as indicators of temperature for the EG stars, but they are very blue. The EG stars of table 8 are hot, and similar to the FB group. Remarks list peculiarities; in the first half we indicate where we have listed a published EG result. The mean $T=36 \mathrm{~km} \mathrm{~s}^{-1}$, the rms $46 \mathrm{~km} \mathrm{~s}^{-1}$; for a Maxwellian distribution of speeds, this corresponds to a $\sigma_{T} \simeq$ $25 \mathrm{~km} \mathrm{~s}^{-1}$. (Part of this dispersion arises from the reflex of the solar motion which has not been subtracted from the observed $U, V, W$.) In consequence, most of these hot white dwarfs seem to be Population I members, descended recently from evolving stars which may include parents of considerable mass. In the Hyades, all parent stars had masses above $2 \mathrm{~m}_{\odot}$. The $W$-components average $-1.6 \pm 0.4 \mathrm{~km} \mathrm{~s}^{-1}$, with a dispersion $\sigma_{W}=+20.3 \mathrm{~km} \mathrm{~s}^{-1}$. However, some stars, if their distances are correct, seem to belong to the old disk (descended recently from stars of $\sim 1 \mathrm{~m}_{\odot}$ ) or even to the halo. A few of these (FB 70, FB 114, EG 63) are DB or DO stars; Eggen and Greenstein (1967) have already noted that a number of helium-rich white dwarfs had high velocities; these included, of course, stars found first in proper-motion surveys. However, some DAwk or DA stars, found by blue color, seem to belong to a different and higher-velocity tail to the low-velocity group. A table of tangential motions for 118 white dwarfs, most selected from proper-motion surveys (Eggen and Greenstein 1967, table 5) shows 65 percent with $v_{T}<40 \mathrm{~km} \mathrm{~s}^{-1}$ as against 62 percent in the presen $\mathrm{t}$

TABLE 8

A. Space Motions of Hot White Dwarfs Found by Color Only

\begin{tabular}{|c|c|c|c|c|c|c|c|c|}
\hline FB & $\begin{array}{l}\text { Color } \\
\text { Survey }\end{array}$ & $\begin{array}{c}U \\
\left(\mathrm{~km} \mathrm{~s}^{-1}\right)\end{array}$ & $\begin{array}{c}V \\
\left(\mathrm{~km} \mathrm{~s}^{-1}\right)\end{array}$ & $\begin{array}{c}W \\
\left(\mathrm{~km} \mathrm{~s}^{-1}\right)\end{array}$ & $\begin{array}{c}\text { Total } \\
V_{T}\end{array}$ & Type & $\theta$ & Remarks \\
\hline 6. & $\mathrm{~F}$ & +11 & -4 & 0 & 12 & $\mathrm{DB}$ & 0.16 & From EG 3 \\
\hline 21. & $\mathrm{~F}$ & +7 & -10 & +3 & 13 & DA & 0.23 & \\
\hline 32. & $\mathrm{HZ}$ & +40 & -15 & -3 & 43 & $\mathrm{DA}$ & 0.26 & 5 EG Hyades mean \\
\hline 67. & TON & +15 & -22 & -11 & 29 & DA & 0.36 & \\
\hline 70 & TON & -74 & -57 & +15 & 95 & $\mathrm{DB}$ & 0.15 & From EG 77 \\
\hline 75 . & & +24 & -34 & -8 & 42 & $\overline{\mathrm{DA}}$ & 0.18 & \\
\hline 88. & $\mathrm{HZ}$ & +31 & -3 & +5 & 32 & DO & 0.10 & From EG 86 \\
\hline 94. & TN & +10 & -5 & -1 & 11 & DA & 0.20 & $\ldots$ \\
\hline 100. & $\mathrm{HZ}$ & +50 & -23 & $-\overline{7}$ & 55 & $\mathrm{DA}$ & 0.23 & . \\
\hline 114 . & $\mathrm{HZ}$ & +3 & -78 & +14 & 79 & DO & 0.10 & $\ldots$ \\
\hline 127. & $\mathrm{HZ}$ & +49 & -102 & -6 & 113 & DAwk & 0.15 & $M_{V}$ poor \\
\hline 145 & & -28 & +12 & -12 & 33 & DAwk & 0.13 & \\
\hline 147. & TON & +8 & -40 & +10 & 42 & DA & 0.18 & $\ldots$ \\
\hline
\end{tabular}

B. EG Hot White DWARfs Found by Color

\begin{tabular}{|c|c|c|c|c|c|c|c|c|}
\hline EG & $\begin{array}{l}\text { Color } \\
\text { Survey }\end{array}$ & $\begin{array}{c}U \\
\left(\mathrm{~km} \mathrm{~s}^{-1}\right)\end{array}$ & $\begin{array}{c}V \\
\left(\mathrm{~km} \mathrm{~s}^{-1}\right)\end{array}$ & $\begin{array}{c}W \\
\left(\mathrm{~km} \mathrm{~s}^{-1}\right)\end{array}$ & $\begin{array}{l}V_{T} \\
\text { Total }\end{array}$ & Type & $\begin{array}{c}U-V \\
(\mathrm{mag})\end{array}$ & Remarks \\
\hline$\ldots \ldots$ & LB & +11 & -4 & 0 & 12 & DB & -1.09 & \\
\hline$\ldots \ldots$ & $\mathrm{F}$ & +7 & -5 & +4 & 9 & DAwke & -1.48 & Old nova \\
\hline$\ldots$ & LB & +6 & -37 & -7 & 38 & DAwk & -1.30 & $\ldots$ \\
\hline$\ldots \ldots$ & $\mathrm{LDS}$ & +19 & -8 & -56 & 59 & $\mathrm{DB}$ & -0.99 & $\ldots$ \\
\hline$\ldots \ldots$ & $F$ & -3 & -1 & +16 & 16 & $\mathrm{DO}$ & -1.65 & $\ldots$ \\
\hline$\ldots \ldots$ & $\mathrm{F}$ & +12 & +3 & -1 & 12 & DO-B & -1.43 & \\
\hline$\ldots \ldots$ & $\mathrm{HZ}$ & -4 & +11 & -1 & 12 & DB & -1.24 & HZ 29, pec. \\
\hline$\ldots \ldots$ & $\mathrm{HZ}$ & 0 & -57 & +9 & 58 & $\overline{\mathrm{DO}}$ & -1.54 & FB 114 \\
\hline $98 \ldots \ldots \ldots$ & $\mathrm{HZ}$ & +10 & -25 & +2 & 27 & DAwk & -1.27 & FB 127 \\
\hline
\end{tabular}


table 8 . But the proper-motion-selected stars had 21 percent in the range $40<v_{T}<80 \mathrm{~km} \mathrm{~s}^{-1}$, similarly the table 8 stars had 29 percent. The statistical error is large, with only six stars in this range. But the comparison suggests that some fast-moving stars (probably old disk and the rarer halo Population II) are found among the present group of white dwarfs. The sdO stars are also suggested to be a mixed population group, but clearly, the hot white dwarfs of table A5 are not a pure halo population group. This is expected, since none are at large $z$-distances. Even with origin from different populations, the evolutionary tracks downward through the sdO star region to the hot white dwarfs may result in the two groups having similar masses, and atomic weights in the core. It is these parameters which control their lifetimes, surface temperatures, and luminosities.

\section{c) Evolutionary Links}

There is so much uncertainty about the $T_{\text {eff }}$ at the hot end of the white-dwarf cooling sequence that we should not overstress evolutionary implications. Lowmass evolutionary tracks through the sdO star region run near $100,000^{\circ} \mathrm{K}$ (Paczyński 1971a). Again, observations from space will be decisive as to typical values of

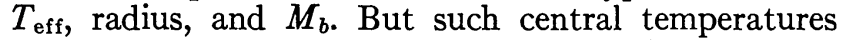
bring the stars close to the region of high neutrino luminosity. If we scale, by the ratio of effective-tocentral temperature found for models of the interior of the hot DA white dwarfs, the hot DO or DAwk stars could have $T_{c} \gtrsim 10^{8^{\circ}} \mathrm{K}, \rho_{c} \simeq 2 \times 10^{7} \mathrm{gm} \mathrm{cm}^{-3}$, and a plasma-neutrino luminosity near $3 \times 10^{3}$ ergs $\mathrm{g}^{-1} \mathrm{~s}^{-1}$ (Clayton 1968). With an isothermal core, a large fraction of the mass is involved since the plasmaneutrino luminosity, near its maximum, is only a slow function of density. Thus we can estimate that the equivalent of half the core mass can radiate neutrinos, i.e., about $6 \times 10^{32} \mathrm{~g}$, yielding a neutrino luminosity near $2 \times 10^{36} \mathrm{ergs} \mathrm{s}^{-1}$. This is 50 times the optical luminosity of the brightest hot white dwarf. The shortening of the early cooling lifetime, when nuclear energy resources no longer exist, would be, of course, dramatic. Using the above model, the thermal energy of nucleons in the relevant part of the core (with mean atomic number $A$ ) is $10^{48} A^{-1} \mathrm{ergs}$, yielding a lifetime of only $1.5 \times 10^{4} A^{-1}$ year. This is comparable to, or shorter than, the lifetime of a nucleus of a planetary nebula. Clearly, the two facets of neutrino emission should be studied together, as well as the optical tracks connecting the sdO star and hot white dwarf regions to establish the maximum $T_{\text {eff }}$ when the degenerate core is nearly exposed. The nuclei of planetary nebulae may become white dwarfs; by the time this occurs the nebulosity will have expanded, with an emission measure too low for detection. A track given by Salpeter (1971, fig. 3) for a carbon-oxygen star of $0.75 \mathrm{~m}_{\odot}$ drops nearly vertically toward the white dwarfs, at $\log T_{\text {eff }} \simeq 5.2$, taking about $6 \times 10^{5}$ years to drop from $10^{3}$ to $6 \times 10^{1} L_{\odot}$, where it is still brighter and hotter than any object we classify as a white dwarf. This time is far longer than the life of any planetary nebula. More massive stars $\left(\sim 1 \mathrm{~m}_{\odot}\right)$ with a small helium envelope $\left(\sim 0.05 \mathrm{~m}_{\odot}\right)$ become much hotter and brighter and evolve more rapidly. It is an important observational problem to determine the maximum temperature of the white dwarfs. Not all need have been nuclei of typical planetary nebulae, or to have dropped vertically through the extremely hot sdO region, during the Harman-Seaton (1964) "stages. Perhaps a link may be found with the sdB and sdO stages at $\log T>4.5$, at least for halo white dwarfs. For the old disk or Population I, the route through the planetary nuclei and the sdO stage is interesting, since the parent stars will have had much larger mass than does the final white dwarf. All such tracks must operate at high $T_{\text {eff }}$, to account for the clear gap between the main sequence and the white dwarfs at $\log T<4.5$. Weidemann (1968) concludes that between 50 and 95 percent of all white dwarfs are not descendants of planetaries.

Another generalization is that mass loss must be substantial. The statistical gravitational redshift of about 80 DA's by Trimble and Greenstein (1972) suggest a mean mass about $0.8 \mathrm{~m}_{\odot}$ for a core of ${ }^{4} \mathrm{He}$, ${ }^{12} \mathrm{C}$ and about $0.7 \mathrm{~m}_{\odot}$ for a ${ }^{56} \mathrm{Fe}$ core. This depends very much on Shipman's calibration of effective temperatures. Extreme values of the acceptable mean mass are $0.65-0.90 \mathrm{~m}_{\odot}$. But Sirius B is the evolved companion of an essentially unevolved A star of $2.3 \mathrm{~m}_{\odot}$; i.e., the white dwarf, now $1.02 \mathrm{~m}_{\odot}$, must have lost two-thirds of its mass. The same is true of the dozen Hyades white dwarfs. If the EHB halo stars produce white dwarfs, their core mass should exceed that of their white dwarf descendants (allowing for some mass loss). This suggests that the lower side of the mass range found by Trimble and Greenstein (1972) should be favored. An independent determination of the gravitational redshifts by Wegner (1974) gives a range $0.6<\mathrm{m} / \mathrm{m}_{\odot}<0.9$.

\section{STARS WITH COMPOSITE SPECTRA}

In the course of the survey for halo blue stars it was noted that many spectra of hot stars showed traces of a diffuse shallow K-line, and sometimes of weak, filled-in metallic lines or the G-band. The $U B V$ colors for these objects were anomalous, located between the main sequence and the blackbody line. Some of these stars have already been described by Greenstein $(1956,1966)$. Since they are not white dwarfs and since most are substantially unreddened, it was concluded that the stars are unresolved doubles in which the cool object contributes at least 10 percent of the blue light. In addition, some relatively high-latitude or high-velocity symbiotic variables were preferentially observed. A sharp interstellar K-line appears in halo B stars, with equivalent width of about $0.20 \AA$ (Greenstein 1968). If the companion is 10 percent as bright as the hot star, its stellar K-line may have $W=2.0 \AA$ yet still only double the strength of the composite observed $W(\mathrm{~K})$. However, recognition of such a cool companion is aided by the broad profile of the K-line in a G star. Highdispersion spectra $\left(18,38\right.$, or $\left.90 \AA \mathrm{mm}^{-1}\right)$ provide quite definite evidence; 190 or $380 \AA \mathrm{mm}^{-1}$ spectra can be ambiguous; the binary nature of fainter stars is definite only if the $U B V$ colors are very abnormal. Synthetic 
composite $U B V$ colors and spectra can be computed for pairs in which one is on the main sequence (type $\mathrm{G}-\mathrm{K}$ ) and the other a horizontal-branch subdwarf or mainsequence B star. These colors resemble the observed ones. Few close or contact pairs occur since few FB stars have emission lines. Emission-line objects are probably old novae or pairs containing a $\mathrm{dMe}$ and a DA white dwarf.

Table A6 gives data on the high-latitude stars with composite spectra; some of these are hardly blue, but contain a blue component. Herbig (1969) discovered symbiotic stars in the galactic bulge, with $M_{V}=-3$. He concludes that they are members of Population II, analogs of nearby high-velocity stars like MWC 603, RW Hya, AG Dra. None of the stars given in table A6 were seen as visual doubles at the 200 -inch telescope; for moderate $\Delta M_{V}$ they must have had separations less than 3"., i.e., less than 1500 a.u. For white-dwarf-dMe pairs like FB 38 (EG 38) or FB 90 (EG 87), the separation must be less than 100 a.u. The systems are probably gravitationally bound; the number of optical pairs expected is small. Figure 15 shows the observed locations of the composite stars in the $U B V$ diagram together with typical computed loci for colors of composite systems. Most are found along a locus requiring that the primary be very blue and faint, i.e., sdB or sdO stars.

The composite stars occupy a distinctive and otherwise empty region of the $U B V$ diagram. A hot subdwarf of large negative $U-B$ with a late-type companion has composite colors indistinguishable from those of a white dwarf or quasar and not far from the blackbody line. Specific assumptions about the cool secondaries are required to define the region of detectably composite colors. Since Population II nonevolved companions are sdG2 or later, the primary must have $M_{B} \geq+2.7$ if the secondary is to produce a 10 percent effect. A subgiant $\mathrm{G}$ or $\mathrm{K}$ would be needed to produce a composite color, if the primary is a horizontal-branch A or late-B star. Population I primaries may have unevolved secondaries of any type because of the variety of their ages, so that the range of possibilities is too large to delimit.

If a companion is $\Delta M_{V}$ magnitudes fainter, and has color differences $\delta(B-V)$ and $\delta(U-B)$ from the primary, the correction to the latter's colors are

$$
\begin{aligned}
& \Delta(B-V) \\
& =\frac{5}{2} \log _{10} \frac{1+\operatorname{dex}\left(-0.4 \Delta M_{V}\right)}{1+\operatorname{dex}\left\{-0.4\left[\Delta M_{V}+\delta(B-V)\right]\right\}}, \\
& \Delta(U-B) \\
& =\frac{5}{2} \log _{10} \frac{1+\operatorname{dex}\left\{-0.4\left[\Delta M_{V}+\delta(B-V)\right]\right\}}{1+\operatorname{dex}\left\{-0.4\left[\Delta M_{V}+\delta(U-V)\right]\right\}} .
\end{aligned}
$$

Once $\Delta M_{V}$ is large, no reasonable $\delta(B-V)$ or $\delta(U-$ $B)$ can produce major effects, and the companion escapes detection. We have computed various cases; the most significant involve a blue, rather faint primary, i.e., a sdO or sdB star. Two types of possible companion are shown for the sdO stars, a sdG2 and a dK; the ultraviolet excess of the sdG2 results in composite colors very close to the blackbody line, as shown in figure 15. The colors of most of our FB composites can be modeled with reasonable accuracy. Since the observed $(U-B)$ 's

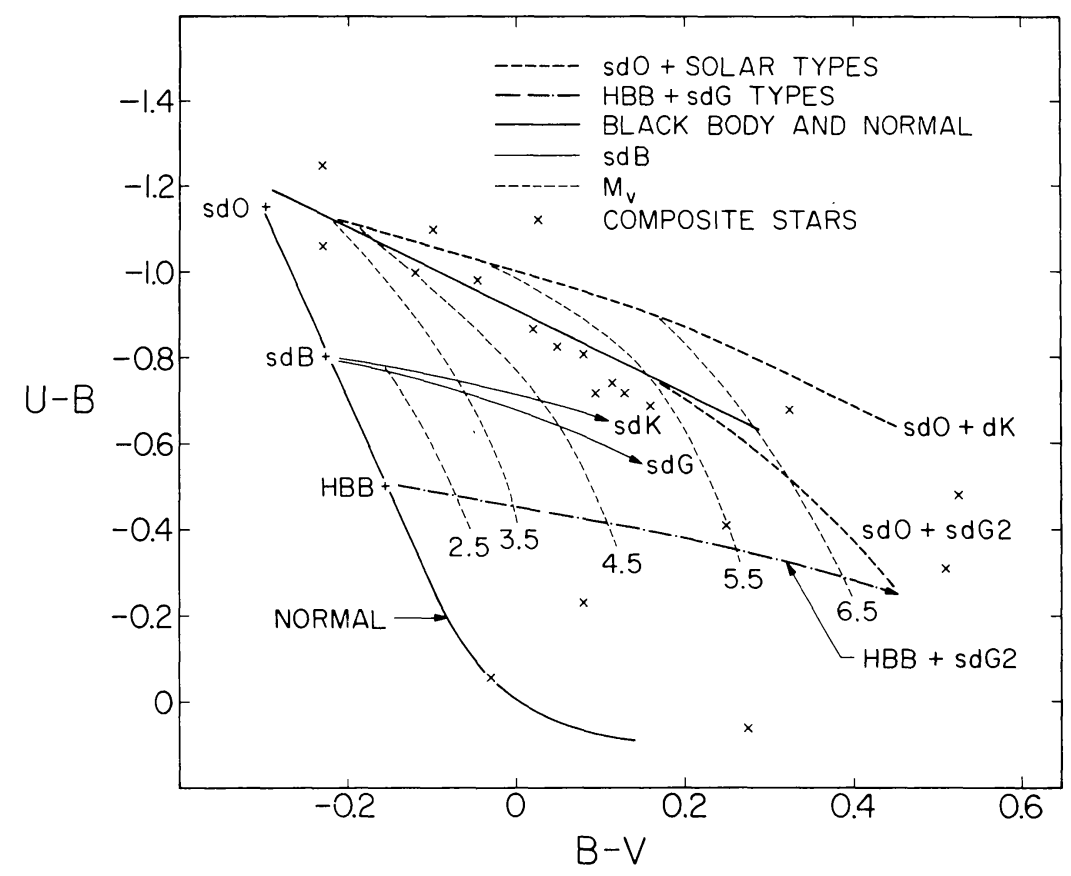

FIG. 15.-The $U B V$ colors of composite stars (table A6) are plotted. The normal and blackbody $U B V$ are heavy solid curves. The predicted composite colors are shown as downward-trending heavy dashed lines, for combinations of sdO colors with various solar-type stars. Thin solid lines show combinations of sdB and solar-type stars; dash-dot lines, HBB. Faint dashed lines are loci of constant $M_{V}$ for the blue components of the unresolved pairs. Most composites are observed near the blackbody line and with the blue star near +5 . 
are quite negative, the primaries must be quite hot, with large negative $U-B$, i.e., a hot $\mathrm{sdB}$ or a $\mathrm{sdO}$ star. Along the trajectories for composite stars involving an sdO star are marked the $M_{V}$ required when the companion is sdG2 at $M_{V}=+4.5$, with colors $B-$ $V=+0.45, U-B=-0.25$; the colors of the $\mathrm{sdO}$ stars are assumed to be $B-V=-0.30, U-B=$ -1.15 . Only slight displacement from this color is produced when $M_{V}(\mathrm{sdO})<+3$. The large displacements such as are observed require $M_{V}(\mathrm{sdO}) \simeq+5.5$ or +6 . The observed mean $M_{V}$ for sdO stars was +3.7 from spectroscopic and kinematic evidence. A pair containing a sdO star and a dK star of $M_{V}=+6.5$, colors $B-V=+0.95, U-B=+0.74$ predicts too bright an ultraviolet to match the observed composite colors. If such a secondary is to produce much effect the primary must have $M_{V}>+5$. A dG5 secondary gives intermediate results.

When the primary has the color of a HBB star $B-V=-0.15, U-B=-0.50$ we obtain results in the lower part of the figure. The trajectories are flat with either a sdG2 or a dK companion; only the former is shown. The rarity of composite stars at small $U-B$, near -0.2 or -0.4 may represent the difficulty of discovery. But most $\mathrm{HBB}$ and sdB stars are too bright compared to plausible Population II companions to have composite color or spectrum. If the pairs are close, there may have been mass exchange, which could have changed the properties of the sdB stars. The location in the $U B V$ diagram of a composite including a $\mathrm{HBB}$ star is quite unusual and would attract attention. Typical composite colors are $-0.1<B-V<+0.2$ and $U-B=-0.4$, i.e., like a reddened $B$ star. The white dwarfs avoid this region, lying near or just below the blackbody line.

The situation is less clear with primaries of intermediate temperatures, below those of sdO stars. A trajectory is shown for an initial color of $B-V=-0.23$, $U-B=-0.80$, combined with a sdG2 star. Such primaries would be sdB stars, with $Q=-0.63, T=$ $19,000^{\circ} \mathrm{K}$; they are intrinsically less common. It is apparent that $M_{V}(\mathrm{sdB})>+3$ is required to move stars far from their initial position. A track for an extreme weak-lined sdK companion, $B-V=+0.95$, $U-B=+0.30$ runs just above that with the sdG star, and requires even lower primary-star luminosities. Expanding equation (14b) for large values of $\Delta M_{V}$ gives

$$
\begin{aligned}
\Delta(B-V)= & 1.086 \operatorname{dex}\left(-0.4 M_{V}\right) \\
& \times\{1-\operatorname{dex}[-0.4 \delta(B-V)]\} .
\end{aligned}
$$

We see that, for a reasonable $\delta(B-V)=1.0$, this reduces to $0.65 \operatorname{dex}\left(-0.4 \Delta M_{V}\right)$. If we note only a color discrepancy larger than $0.10 \mathrm{mag}$, we are limited to $\Delta M_{V}<2.0$. A blue Population II star with a companion at the turnoff point is noticed as composite only if $M_{V}>$. 2.5. The situation is worse in $\Delta(U-B)$, since $\delta(U-$ $V)>\delta(B-V)$. Horizontal-branch stars cannot be much affected, except if the companion is itself beginning to evolve. Most of the composites we have found are therefore among the faintest and hottest of the halo stars.

Our figure 15 displays that the composites are a highly selected group, with the observed points on plausible tracks when the blue star is a very hot, intrinsically faint star. Stars like FB 18, FB 27 might not have been noted if the errors in their colors were slightly above average. The white dwarfs with composite spectra plotted, FB 24, FB 38, FB 90, FB 173, had been previously found (Eggen and Greenstein 1965). The particularly interesting composite FB 33, FB 34 is the nucleus of a planetary nebula, NGC 1514, and had been observed because of its apparently A-type. The two stars are shown separately as deconvoluted by Greenstein (1972) and are listed separately in other tables. The summed colors lie very near the normal $B-V, U-B$ relation, but the star shows strong He II absorption. Of the high-velocity symbiotic variables, only FB 110 is plotted; FB 171, FB 185 are too red. Allowance for such preselection among $21 \mathrm{FB}$ stars in table A6 reduces the percentage of composites from 11 to about 6 percent of the FB stars observed. But the very stringent limitation on $\Delta M_{V}$ represented by the equations and shown in figure 15 suggests that binaries are very common among the FB stars. For example, let us assume that companions in the 3-mag range, e.g., $+3<M_{V}<6$ are always detectable. In the range $+6<M_{V}<+10.5$ (dK and early $\mathrm{dM}$ stars) the normal luminosity function predicts that three times as many undetectable as detectable companions to the evolved blue stars exist. Thus about 24 percent of the halo stars should have companions, close enough to the Population I frequency of binaries to suggest that wide binaries are equally common in both populations. There seems to be a low percentage of rapidly revolving, spectroscopic or contact binaries in Population II; novae are old-disk in nature. One-third of the stars in table A6 have emission lines, but obviously there has been selection in favor of such objects.

The kinematics of this group is largely unexplored. If we omit the white dwarfs, only FB 10, FB 18 have $\mu>0$. 020 ; another four have 0 ".006 $<\mu<0$. 015 . These unsatisfactory statistics are accompanied by a wider distribution in galactic latitude than our other groups. The reduced proper motions give $\left\langle M_{V}\right\rangle=$ $+13.0-5 \log \left\langle V_{T}\right\rangle$. If the total $M_{V}$ is, from the spectroscopy, $+3.5,\left\langle V_{T}\right\rangle$ is $80 \mathrm{~km} \mathrm{~s}^{-1}$. With the modest data on radial velocities shown in table A6, $\sigma= \pm 37$ $\mathrm{km} \mathrm{s}^{-1}$, we would find $\left\langle M_{V}\right\rangle \simeq+4.5$.

\section{EVOLUTIONARY IMPLICATIONS}

\section{a) The Areas Occupied in the $H-R$ Diagram}

It would not be wise in a paper full of technical and observational detail to assume that we are in the best position to explain the somewhat bewildering array of stars we have uncovered. Statistically, we overemphasize the lower-left quadrant of the Hertzsprung-Russell diagram, which is full of pathological specimens of late stages of evolution approaching the white dwarfs. In figure 16, the various groups of stars are shown in the 


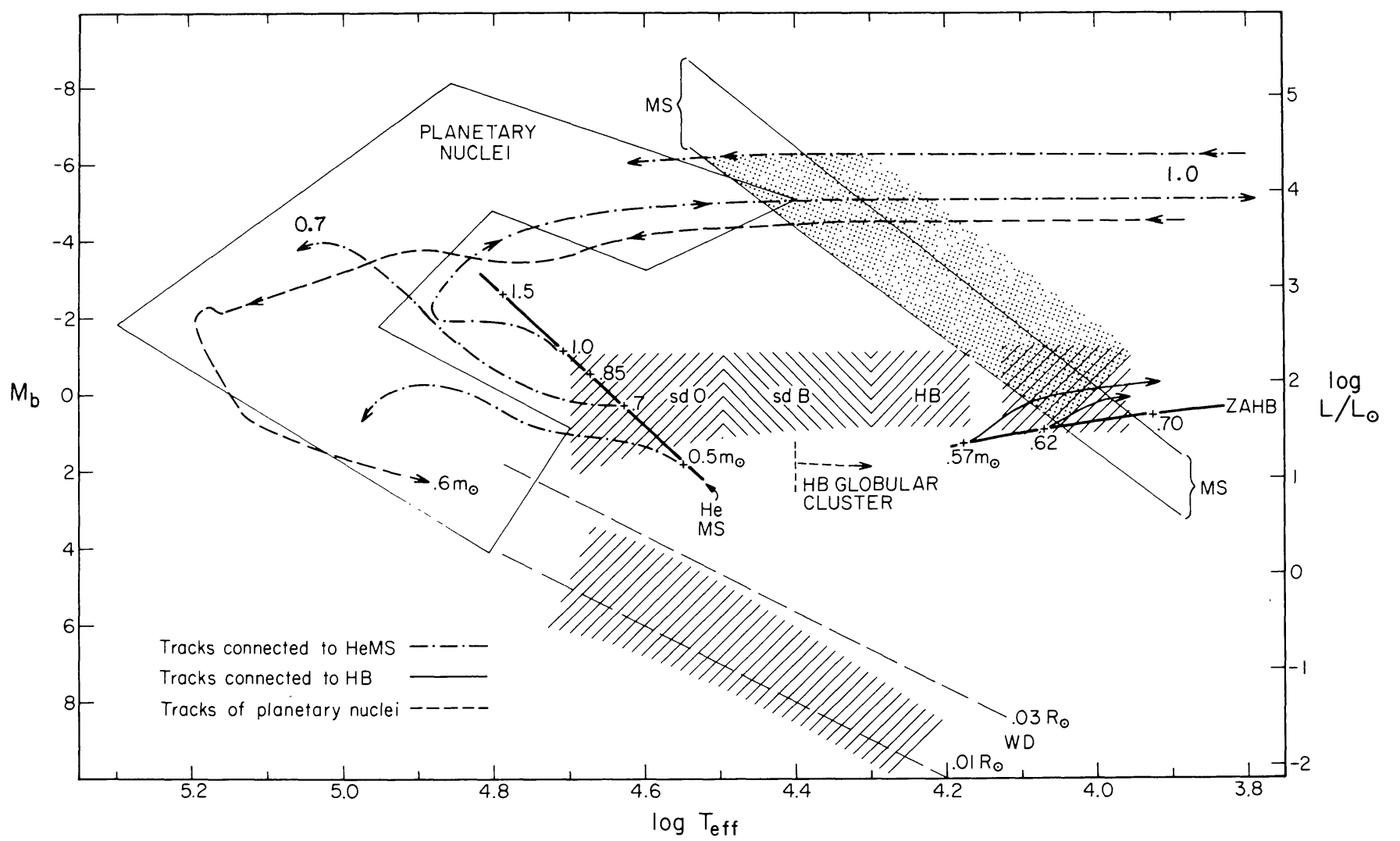

FIG. 16.- Shaded and stippled areas are occupied by stars of the FB lists; the normal stars lie near to or above the main sequence (MS). Planetary nuclei (from O'Dell 1968) lie in the enclosed area at the upper left. Evolutionary tracks are shown with arrows from the zero-age horizontal branch, labeled by total masses. The normal high-temperature limit of globular-cluster horizontal branches is shown. The helium main sequence (He MS) is shown labeled with masses and with evolutionary tracks. Note the large area near $M_{b}=0, \log L / L_{\odot} \simeq 2$, occupied by the extended horizontal branch, without evolutionary tracks.

$\left[M_{b}\right.$ (or $\left.\left.\log L / L_{\odot}\right), \log T_{\text {eff }}\right]$-plane. Areas in which our FB stars are found are shaded or stippled. We add an open area, representing the bright, very hot planetary nuclei from O'Dell (1968). Also sketched is the standard hydrogen main sequence (MS) including a plausible range above it (i.e., some evolution) from Iben (1967b). In § VIII $c$ we discussed the problems raised by highlatitude stars of normal mass and composition. The area within which white dwarfs of between 0.01 and 0.03 $R_{\odot}$ are found is shown. These radii are reasonable for zero-temperature degenerate stars with helium (or heavier) cores, and the hydrogen envelope has negligible effect on $R$. White-dwarf masses with such radii lie within limits compatible with the observed gravitational redshift, and are near the mass used for the horizontal-branch stars ( $\$$ IX and XI).

Plausible evolutionary tracks populate the region occupied by the apparently normal (table A1) stars in figure 16. We have earlier indicated the possibility that we have somewhat overestimated some luminosities of normal stars from (a) a systematic error in spectrophotometry, (b) high helium abundance, or (c) use of masses from the normal mass-luminosity relation. The major difficulty remaining is that we require high- velocity ejection for many stars, by a mechanism such as Blaauw (1961) proposed. The evolutionary problems are discussed more fully in $\S$ VIII.

For the white dwarfs (table A5) we see no difficulties except that our selection process emphasizes very blue objects, and therefore a number of objects are classified as white dwarfs which are hotter and therefore more luminous than are the familiar DA stars. The existence of DO stars with high color temperature and strong $\mathrm{He}$ II lines clearly suggests that we are seeing short-lived, early cooling stages of stars with helium atmospheres and possibly heavy-element cores, i.e., the exposed centers of red giants. Since sdO stars are often heliumrich, a crossing point may exist at some unknown high $T_{\text {eff }}\left(\log T_{\text {eff }}=4.8-5.0\right)$ where such stars of 0.01 or $0.03 R_{\odot}$ would be spectroscopically and probably structurally indistinguishable. Since we cannot determine $T_{\text {eff }}$ with any accuracy above $\log T_{\text {eff }}=4.6$, the apparent gap is an artifact.

We find no stars between the white dwarfs and the extended horizontal branch in the region $\log T_{\text {eff }}<$ 4.50, as shown from detailed examination of earlier diagrams (figs. 7 and 8). None of the white dwarfs are variable or have magnetic fields. They may arise from 
either the halo, the old disk, or Population I, and as long as the masses are less than about $1.2 \mathrm{~m}_{\odot}$ they seem not to offer any problem.

In figure 16 we plot an assortment of theoretical loci and evolutionary tracks that relate to FB stars. The zero-hydrogen stars with helium and some metals are shown labeled as the helium main-sequence, with masses as given; pseudo-evolutionary tracks are computed by Biermann and Kippenhahn (1971) and evolutionary tracks by Paczyński (1971a). We show the latter's tracks, including neutrino losses. Note that the helium stars of masses $0.5,0.7$, and $0.85 \mathrm{~m}_{\odot}$ evolve upward but only to the left, and presumably become very hot white dwarfs when their small nuclear energy is exhausted. However, a helium star of $1.0 \mathrm{~m}_{\odot}$ has a horizontal branch moving rapidly to low temperatures at nearly constant $\log L / L_{\odot}=+4.0$, and then returning towards the planetary nuclei. This may be relevant to some hot carbon or helium supergiants and to the cool $\mathrm{R} \mathrm{CrB}$ variables. Some of the peculiar bright stars of table A1 may be in such a short-lived state. But, unfortunately, a "red giant" with carbon core and helium envelope is too luminous to be relevant to our horizontal-branch problem. This is unfortunate since the $\log \left(L / L_{\odot}\right)$ $\left(\mathrm{m} / \mathrm{m}_{\odot}\right)^{-1}$ value is 1.49 for the unevolved helium ZAMS star of $0.5 \mathrm{~m}_{\odot}$ and 1.95 at $0.7 \mathrm{~m}_{\odot}$, just in the observed range (1.83) of our extended horizontal branch. However, it rises to 4.0 for $1.0 \mathrm{~m}_{\odot}$. Perhaps a clue exists here suggesting investigation of models of evolving helium stars, with carbon core and hydrogen envelope. Trimble and Paczyński (1973) find that helium red giants, i.e., evolutionary tracks toward the right, can be produced for $0.85 \mathrm{~m}_{\odot}$ stars if the neutrino emission is increased for nonphysical reasons by 10 . These tracks are still at too high an optical luminosity to be interesting. Static, nonevolutionary models also avoid the region of interest. In general, as the ratio $q$ of the mass of the core to the total mass increases, the star moves very rapidly to the right and becomes too cool to be of interest for us.

Stars like the bright $\mathrm{B}$ and $\mathrm{O}$ stars of the globularcluster population lie well below the main-sequence; some are listed in table A1 and discussed in \$ VIII. A few more with negative $B-V$ have been discovered by Zinn, Newell, and Gibson (1972). They clearly lie below the $\log L / L_{\odot}=4$ track of figure 16 and above the horizontal branch. Their evolution may be connected with that of the asymptotic horizontal-branch red giants with unexpected compositions (Strom and Strom 1971). However, their luminosity is above the "suprahorizontal branch" shown in Iben (1971).

\section{b) The Horizontal Branch}

Computation of theoretical evolutionary tracks for horizontal-branch stars has been so extensive that we cannot here review its application to our observations. Iben (1971) and Rood (1973) give extensive reviews; the general conclusion is that the evolution from the redgiant phase into the horizontal branch is adequate to explain most of the cooler globular-cluster horizontalbranch stars. Gross (1973) considers the effects of $\mathrm{He} / \mathrm{H}$ ratio, metal abundance, and core mass to total mass.
Rood (1973) predicts cluster horizontal branches with a spread in masses and with different $\mathrm{He} / \mathrm{H}$. We sketch the zero-age horizontal branch (ZAHB) from Iben (1971), for metal-poor stars with masses as shown. The core helium burning and shell hydrogen burning produce evolution predominantly to the right. With reasonable spread in masses, core masses and $\mathrm{He} / \mathrm{H}$, the evolution for the HBA and late $\mathrm{HBB}$ stars can be understood. Our luminosities and masses for field stars agree suffciently well with that contemplated for the clusters. Rood's synthesized diagrams reach to $\log T_{\text {eff }} \simeq 4.30$. The ZAHB models by Gross (1973) reach somewhat higher temperatures, up to $\log T_{\text {eff }}=4.38$, but only with a largely "core" star, i.e., $q=M_{c} / M>0.95$. Furthermore, Gross finds the downward trend in luminosity to be even stronger than Iben's. He gives stars of $\log T_{\text {eff }}=4.35$ as in the range $+1.5<M_{b}<$ +2.5 . We do not feel that the hotter HB stars in clusters, or among our field stars, have yet been explained. The width of the HB, the luminosity, and the stellar mass seem reasonable. Stars with $q \rightarrow 1.0$ can live only a short time, before traveling to the right.

We must now examine the near absence of bluer stars, such as sdB or sdO stars in the globular-cluster branches. In figure 6 we used the photoelectric data of Sandage $(1969,1970)$ on the best observed clusters, M3, M13, M15, M92. His moduli were determined by fitting the clusters to the turnoff points, allowing for the effects of metal deficiency on G-star colors. Allowing for observational errors in the moduli which may reach $\pm 0.2 \mathrm{mag}$, it is still found that near $B-V=0.00$ the cluster horizontal branches spread over $0.7 \mathrm{mag}$, have slightly different downward slopes for the blue stars, and essentially stop by $Q=-0.65$, i.e., $\log T_{\text {eff }}=$ 4.30. Sandage's observations of the only known hot sdB star in M13 show a spread which Sandage kindly estimated from his original records. Its error ellipse in figure 6 embraces the temperature and gravity of Sargent and Searle (1971). Inspection of the EHB we sketch in figure 16 shows that if brighter $\mathrm{sdB}$ or $\mathrm{sdO}$ stars are yet to be found in globular clusters, blue stars about 2.5-4.0 mag below the RR Lyrae variables must be searched for. Note the sdB star in M13 has special importance in that it appears to lie 1 mag above our EHB. This is probably near or beyond the limit of observational error; if it represents a different evolutionary stage there is, in fact, a genuine break between the cluster HB stars and the $\mathrm{sdB}$ or sdO stars. The sense (see table 2) is that its mass is larger than that of an average $\mathrm{HB}$ star for the $\theta, g$ observed. It is worth remembering that M13 seems to have a peculiarly high $M_{V}$ for its two RR Lyrae variables (Sandage 1970, fig. 18 ), which is in the sense of larger mass required.

Evolutionary HB tracks have, so far, not reached the blue sdB and sdO stars, because they turn back redward or have vertical excursions (Rood 1973; Gross 1973). Demarque and Mengel (1971) give an upwards evolution at $0.50 \mathrm{~m}_{\odot}$, with $q=0.77$. It may be argued that a real difference exists between field and globularcluster populations, or even within the field stars, between sdO, sdB, and HBB stars. We do not believe it 
incorrect, however, that we have linked the sdB and HBB stars, based on the nearly identical values (within $0.1)$ of $\left\langle\log g \theta^{4}\right\rangle$ they display.

Abell (1966) used the surface brightness and size of the old planetary nebulae he had found to derive properties of the central stars. He gives bolometric luminosities derived on the basis of an optically thin model for the nebulae. He finds minimum Zanstra temperatures for the central stars, and minimum log $L / L_{\odot}$ in the range $0.6-2.5$. Some may vary in light. This statistical method leads him to suggest that his planetary nuclei are an extension of the horizontal branch from $\log T_{\min }=4.3-5.4$, to merge with the white dwarfs. Most of his stars have $-1<M_{b}<+3$, and could, in fact, be near the EHB at very high $T_{\text {eff }}$. Such stars lie, in fact, near the helium main sequence and on some of the evolutionary tracks shown in figure 16. His results strongly suggest the importance of maintaining an open mind on possible evolution at very high $T_{\text {eff }}$.

Newell (1973) pointed out that the frequency distribution of $U B V$ colors suggests that certain colors were infrequent. He plots the cumulative number, $N(Q)$, of objects redder than a certain value of $Q$; he finds what he views as statistically significant gaps, where the slope of $N(Q)$ changes, or stars are rare or absent. One gap is at $-0.245<Q<-0.325$, the other at $-0.675<$ $Q<-0.720$. He does not find them as clearly visible in the globular clusters; the first is weakly indicated, the second, however, is at the blue end of the cluster horizontal branches. We have reexamined our data on field EHB stars (HB, sdB, and sdO stars, tables A2,
A3, A4) with results shown in figure 17. The middle curve (filled circles) contains FB normal and EHB stars. It shows essentially no gap; in fact, the slope is quite normal through Newell's first gap. The upper curve (open circles) shows counts only of the EHB stars. It demonstrates beautifully that halo FB stars are rare in the range $-0.29 \leq Q \leq-0.42$, with none of our stars lying in that interval. This is near to and wider than Newell's gap 1 . However, with our larger number of very hot stars we do not see any convincing evidence for Newell's gap 2, although there is a decrease in the slope. To ensure that gaps are not a result of the method of calibration of stars of the $U B V$ photoelectric system, we derived $Q$ from $350 U B V$ colors from the Blanco et al. (1968) catalog of bright B stars. These were selected at random from the first 5400 entries in that catalog. Figure 17 shows the distribution of $Q$ for these normal Milky Way B stars. For these, $N(Q)$ is smooth with only gentle changes in derivative. Many of the Population I stars were highly reddened, so that the reddening law is tested by this smoothness. No extremely blue stars exist unless all the hottest Population I stars have an abnormal reddening. Only 22 percent of the Population I had $Q \leq-0.80$, as compared to 58 percent of the field EHB stars. This figure is somewhat high since we include Abell stars which have a high discovery probability. We have observed selectively the bluer candidate stars from colorimetric surveys at the galactic poles, but it still is clear that halo stars can be intrinsically hotter than main-sequence stars. The reason, presumably, is that the upper limit to effective

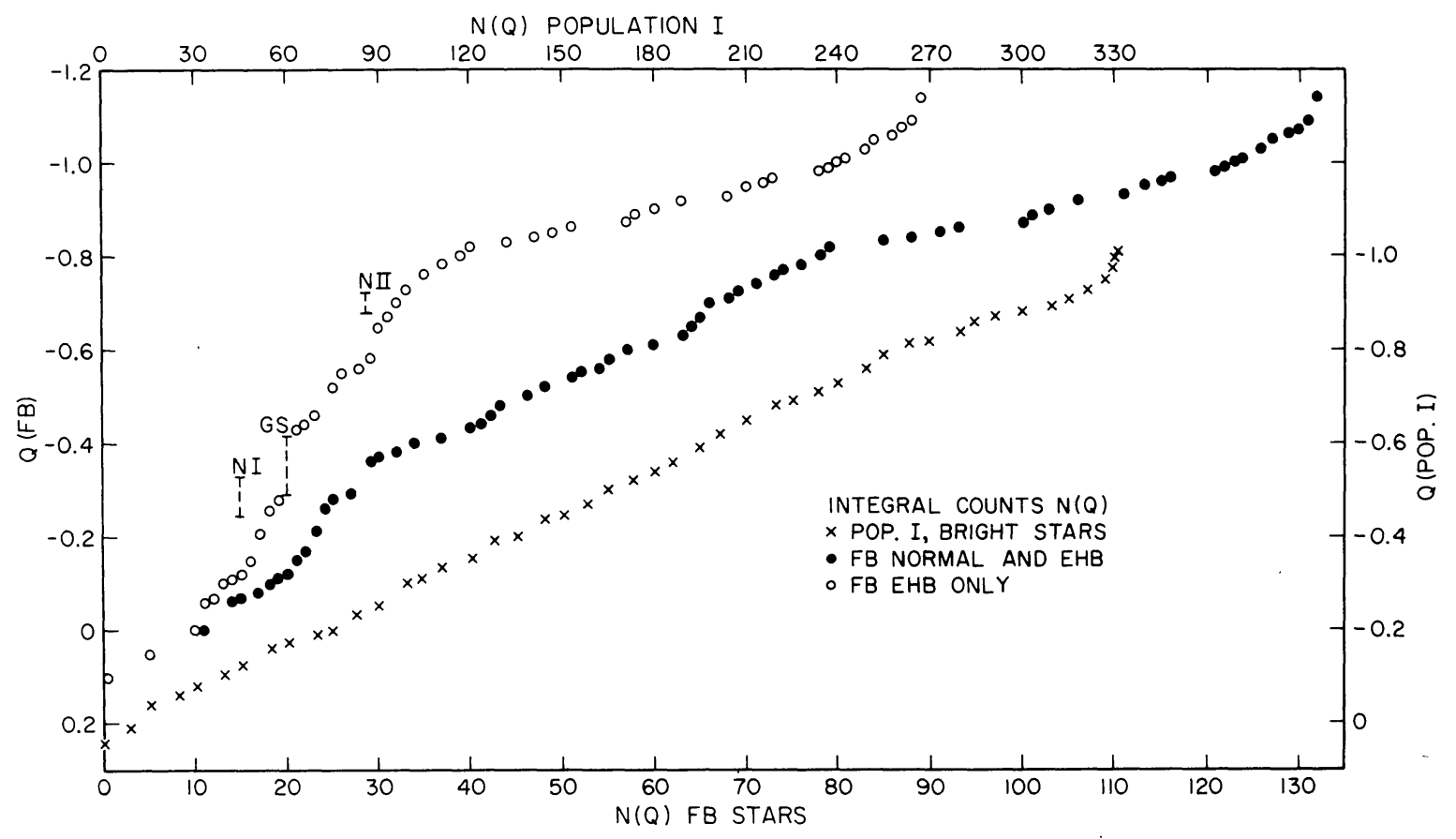

Fig. 17.- The cumulative number of stars bluer than a given color, $Q$ is $N(Q)$. The crosses on the lower, smooth curve refer to normal bright field stars of Population I (upper and right-hand scales). The open circles are the FB, HB, sdB, and sdO stars, and demonstrate that a gap exists (labeled GS), $-0.27<Q<-0.42$. Newell's gap 1 is shown, labeled N I. The filled circles show $N(Q)$ counts of the EHB plus the high-latitude normal stars, and are almost completely without a gap. Newell's gap 2, N II, at higher temperature is not conspicuous. A larger fraction of high-latitude stars reach high temperatures than in Population I. 
temperature in a massive star is set by stability considerations, i.e., an uppermost stable mass, or by radiation pressure. The former no longer is relevant in Population II and the high surface gravity, in sdO stars, for example, permits very high temperatures. Presumably, for homologous stars, raising $T_{\text {eff }}$ raises the central temperature and ultimately the process is limited by neutrino losses. Thus, we may be pleased that we find so many excessively hot objects, even if we find no evolutionary track clearly leading to them.

The gap labeled 1 in Newell's (1973) figures 2 and 3, is shown in our figure 16, with our limits $4.11<\log$ $T_{\text {eff }}<4.17$. Globular-cluster stars and HBB stars populate well the region to the left. Our poor statistics do not provide us with evidence for Newell's gap 2 $\left(4.313<\log T_{\text {eff }}<4.343\right)$. We found one star in his interval of 0.045 in $Q$; the range $-0.50<Q<-0.78$ includes 14 stars, and gives an expectation of $2.25 \pm$ 1.50 stars in gap 2 . For gap 1 (for which he has relatively more stars), we expect $4.55 \pm 2.13$ stars, and observe none. Such significance tests may be invalid when proper account is taken of the effect of our selection of the "interesting" interval. The face that cluster stars extend to color temperatures of $Q=-0.8$, i.e., log $T_{\text {eff }}=4.40$ carries their temperature well into the sdB region, although an insufficient number of cluster-star spectra have been classified quantitatively to establish their identity with the field sdB.

If the halo field stars resemble clusters, and if the two sdB stars in M13 are typical, we see no reason for not viewing the hotter $\mathrm{sdB}$ and $\mathrm{sdO}$ stars as related types. The HBB and cooler sdB stars all suffer $\mathrm{He} I$ line weakness in varying amounts; sdO stars have strong helium $\mathrm{He}$ II and He I lines; one possibility is that the extension of cluster HB sequences to higher temperatures is accompanied by the appearance of helium at the surface. From the conventional HB models, the core mass approaches the total mass, and both are small $\left(\simeq 0.42-0.45 \mathrm{~m}_{\odot}\right)$ at very high $T_{\text {eff }}$. If helium flash or related instabilities occur in a star with $q$ near unity, some unexpected, short-lived excursions towards the blue may be expected. If ${ }^{4} \mathrm{He}$ to ${ }^{12} \mathrm{C}$ burning has occurred it should be remembered that a well-mixed carbon low-mass star lies at the far upper left $(\log T>$ $5.0)$ : of figure 16.

If a completely different evolutionary status, mass, and composition is required to explain the sdB and sdO stars, note that it will be necessary to explain why $\log g \theta^{4}$ for these is so close to its value for HBB stars. There has been no prior selection for the sdB stars, so that the numerical coincidence in $L / \mathrm{m}$ is remarkable. Among the sdO stars we have emphasized high-g objects, since the planetary nuclei (some of which have low $g$ ) are so rare in space, and since our technique fails for high $T_{\text {eff }}$. Models are lacking, and the hydrogen lines not measurable. Nevertheless, at least half of the sdOs we studied lie near the line $\log g \theta^{4}=2.35$, i.e., at the same bolometric luminosity (for constant mass) as the RR Lyrae variables, and the HBA and HBB stars that are undoubtedly members of globular clusters. Of course, we do not obtain $L$, but from our $L / \mathrm{m}$ value observed spectroscopically we derive $\log L / L_{\odot}$. The change in $\log L$ is only 0.18 if we increase the mass used in our analysis from $0.66 \mathrm{~m}_{\odot}$ to $1.00 \mathrm{~m}_{\odot}$; larger increases are implausible for sdOs of the old disk or for the highvelocity halo sdOs.

\section{THE HELIUM SURFACE ABUNDANCES}

\section{a) The Helium Deficiency is not Cosmological}

Early formation of helium with nearly the same abundance $(Y \sim 0.2-0.3)$ as in young stars is often blamed on a cosmological event. Special variant cosmologies may reduce the early helium of old stars. It is therefore a challenge to the observer to produce genuinely helium-poor stars. By 1956 a sufficient number of globular cluster and field HBB stars had been observed to demonstrate a low surface abundance of helium. The results were discussed by Münch (1956), Greenstein (1966). Illustrations are given in Greenstein and Münch (1966) and Greenstein (1960). The globularcluster stars directly proved that hot stars with weak helium lines existed, paralleling metal deficiencies common in sdG stars of the halo Population II. Sargent and Searle (1968) found a helium deficiency of a factor of 10 in some Feige stars $(Y=0.03)$. Since unusual stages of evolution were involved, which might have had surfaces contaminated by helium produced by hydrogen burning, the low helium abundance had serious cosmological implications.

Since about 10 percent of the hot white dwarfs show only $\mathrm{He} \mathrm{I}$ lines (Greenstein 1969, table 3), and since strong $\mathrm{He} \mathrm{I}$ and $\mathrm{He}$ II lines were found in subdwarf $\mathrm{O}$ stars, it was clear that stellar He synthesis occurred, and the evidence for this ultimately appeared in such stellar atmospheres. However, the commonest white dwarfs have hydrogen spectra. We see into only a few grams per square centimeter of a stellar atmosphere. It could be dangerous to deduce the primeval composition of a star from the surface composition. A troublesome question existed in the early years of this problem, i.e., are $U B V$ colors, in fact, reliable indicators of temperature? Almost all recent narrow and broad-band photometry, multichannel spectrophotometry, and observations from satellites, however, have shown that the stellar color-temperature scale and model atmospheres agree fairly well. Further, predicted non-LTE and LTE continua show only small differences. Infrared anomalies found for emission-line B stars are not relevant; interstellar or intrinsic reddening seems small for the halo. Thus we no longer expect large errors in temperature for the halo or globular-cluster hot stars. Further, the high gravities suggest that non-LTE effects should be small. Line broadening only strengthens lines relative to hot main-sequence stars, and effects of dilution of radiation do not occur. In résumé, a large surface helium deficiency exists in most $\mathrm{HBB}$ and sdB stars $(Y \simeq 0.03)$. But the sdO stars so far analyzed have $Y$ from 0.4 to 0.9 (Peterson 1969; Tomley 1970). The star FB 103, intermediate between sdB and sdO stars shows, for a sdB star, relatively strong $\mathrm{He} \mathrm{I}$ and $\mathrm{S}$ III lines. The HBB star FB 137 also has relatively strong $\mathrm{He} \mathrm{I}$, and 
P II lines (Sargent and Searle 1967). We note further that the types of peculiarities commonly seen in Ap or magnetic stars even on low-dispersion classification-type spectra (Morgan, Keenan, and Kellman 1943) would have been noted in many of our halo stars. Perhaps 10 percent of Population I A or B stars show such peculiarity. Given the overall weakness of metallic lines, it is possible that many so-far undetected types of Population II Ap or Bp stars with very weak lines but anomalous abundances will be found. He I lines are weak in some Population I Bp stars, but helium excess is seen for hotter stars (Osmer and Peterson 1974). We believe that surface helium weakness is correlated with peculiar element strength, and that both are purely atmospheric phenomena. Excellent reviews of the current evidence on the $\mathrm{He} / \mathrm{H}$ ratio by Danziger (1970) and by Searle and Sargent (1972) demonstrate no truly low $\mathrm{He} / \mathrm{H}$ abundance anywhere; the $\mathrm{G}$ subdwarf masses and the RR Lyrae pulsation theory require an essentially normal initial helium content. Searle and Sargent (1972) feel, probably correctly, that the atmospheric helium content is not representative of the entire star.

\section{b) Gravitational Separation and Mixing}

The hypothesis that gravitational diffusion separates light and heavy elements in the white dwarfs has been quite important and successful theoretically (Schatzman 1958) and is required by observed extreme metal deficiencies at the surface. Applied by Greenstein, Truran, and Cameron (1967) to the halo B stars, it may be the source of the $\mathrm{He} / \mathrm{H}$ deficiency. The atmospheres must be very stable because of absence of rotational mixing or convection (or possibly the presence of magnetic fields). Michaud (1970) added the effects of radiation pressure on selected metallic ions, in an otherwise stable atmosphere, to explain various types of Ap or Bp stars.

It was noted by Searle and Sargent (1967) that Population I Ap and Bp stars were concentrated in the region defined by $0.25<\theta<0.60$ and $3.0<\log g<$ 4.4. They pointed out that the low-temperature boundary of this region could arise from the onset of a shallow hydrogen convection zone; above the high-temperature boundary there was no $\mathrm{H}$ or $\mathrm{He}$ surface convection zone. At fixed $T_{\text {eff }}$, the electron pressure for partial ionization of $\mathrm{He}$ would probably depend on $g^{1 / 2}$. For EHB stars temperature boundaries shift to higher $T_{\text {eff }}$. Lacking surface convection, or rotation, only slow mixing would occur, permitting radiation pressure to compete with gravity, to support small amounts of certain "peculiar" elements, like $\mathrm{P}, \mathrm{Si}, \mathrm{Ga}$, or permit gravitational diffusion.

We have inspected a large number of models with interesting values of $\theta$ and $g$, to see what phenomena occur which could favor (or inhibit) stable layering of the surface of a nonrotating star As far as we know, rotation is small or absent in halo B stars, emission lines are absent (e.g., no shells); no definite proof exists of the presence of general magnetic fields. As was already pointed out in table 7 of Mihalas (1965), the hot main-sequence stars at $\log g=4.5, \theta<0.45$ de- velop an ionization zone $\left(\mathrm{He} \rightarrow \mathrm{He}^{+}\right)$, which lasts till $\theta<0.25$, where the second helium ionization appears $\left(\mathrm{He}^{+} \rightarrow \mathrm{He}^{++}\right)$. His figure 6 shows the depths of convection at the relatively low $g$ he studied. Inspection of models collected or computed by Shipman show quite strange, nonmonotonic behavior of the ratio $\mathrm{He}^{++}$ $\mathrm{He}^{+} / \mathrm{He}$ as a function of optical depth. Several convective zones may be present, with a ratio of convective to radiative flux near $10^{-4}$. The convective velocities are near $10^{2}-10^{3} \mathrm{~cm} \mathrm{~s}^{-1}$; with a scale height of $10^{8} \mathrm{~cm}$, the time scale for mixing by a random walk is short, about $10^{10} \mathrm{~s}$. A survey of available models, at $\log g=5$ and $\log g=6$, gives the following dependence of convective zones on temperature.

1. At $12,000^{\circ} \mathrm{K}$, the $\mathrm{H}$ convection is at the surface; the He ionization is deep, begins at $\tau=5$ for $\log g=5$, at $\tau=30$ for $\log g=6$.

2. At $17,500^{\circ} \mathrm{K}$, the $\mathrm{He} \rightarrow \mathrm{He}^{+}$zone is near $\tau=1$. 3. At $20,000^{\circ} \mathrm{K}$ and $25,000^{\circ} \mathrm{K}$, the $\mathrm{He} \rightarrow \mathrm{He}^{+}$zone is at the surface, and the $\mathrm{He}^{+} \rightarrow \mathrm{He}^{++}$zone is very deep.

4. At $30,000^{\circ} \mathrm{K}$, all $\mathrm{He}$ is at least once ionized, with the $\mathrm{He}^{+} \rightarrow \mathrm{He}^{++}$transition deep, at $\tau=8$ for $\log g=5$ and $\tau=15$ for $\log g=6$.

5. By $37,500^{\circ} \mathrm{K}$, at $\log g=6$, the $\mathrm{He}^{+} \rightarrow \mathrm{He}^{++}$zone approaches the surface, $\tau=0.3$. At higher $T$ no important partial ionization zone remains.

6. Assume that when $\mathrm{He}^{+} \ll \mathrm{He}^{++}$convection can no longer be driven. Only $\log g=4.5$ models are available for very high $T$ (Mihalas 1972a); but for any $T>$ $30,000^{\circ}$, helium is doubly ionized at $\tau>0.5$. Thus deep convection will cease somewhere between $30,000^{\circ}$ and $37,500^{\circ} \mathrm{K}$ at high $g$.

We lack high-gravity models for further study of the change from situation (4) to (5), but it is clear that a very important change does occur about $25,000^{\circ} \mathrm{K}$. It is also possible that the second helium ionization may create an unstable situation near $30,000^{\circ} \mathrm{K}$, so that mixing, and specifically, mixing of helium from large $\tau$ to or near the surface occurs. Somewhere hotter, no convection is expected. If there had been earlier stratification separating $\mathrm{He}$ from $\mathrm{H}$, the gradient of molecular weight could further stabilize the atmospheres; low-He content provides less driving force for He convection.

Another phenomenon of interest occurs at high $T$ namely, the increase of continuum radiation pressure on all the gas, not only on peculiar rare elements. At these high temperatures, the electron scattering is a major opacity source and does not depend on $T$. We evaluated $p_{r} / p_{g}$ at $\tau=1$ in some high- $g$ models which Shipman had computed. At $30,000^{\circ} \mathrm{K}$, the ratio drops from 0.21 $(\log g=4)$ to $0.013(\log g=6)$; at $50,000^{\circ} \mathrm{K}$, it is 0.62 $(\log g=4), 0.060(\log g=6)$ and $0.008(\log g=8)$. Note that $p_{r} / p_{\theta}$ would be small but appreciable at $30,000^{\circ} \mathrm{K}, \log g=5$ and even smaller at $50,000^{\circ} \mathrm{K}$, $\log g=6$. Thus, along the EHB, in spite of increasing $g$ and $p_{g}$, radiation pressure is small but might conceivably start an instability arising from negative gaspressure gradients.

The observations suggest that the low-helium surface phenomenon extends up through the sdB stars, i.e., to 
$T_{\text {eff }}<31,000^{\circ} \mathrm{K}$. There is a possible instability arising from second ionization of helium. By $37,500^{\circ} \mathrm{K}$ this transition has reached close to the surface. The sdO stars are mostly above the $T_{\text {eff }}$ at which the $\mathrm{He}^{+} \rightarrow$ $\mathrm{He}^{++}$transition occurs at moderately large $\tau$. Along the $\mathrm{EHB}, \log g$ increases rapidly at such temperatures. The radiation pressure (for a fixed opacity, e.g., electrons) does not increase with temperature, especially with $g \theta^{4}$ nearly constant. Thus, if a star evolved to a hotter stage, once it has passed $37,000^{\circ} \mathrm{K}$ it is unlikely that any atmospheric phenomenon will bring helium to the surface. Between $30,000^{\circ}$ and $37,000^{\circ} \mathrm{K}$ there may be a driving force (convection). Above such $T$, the driving force bringing helium to the surface would more probably be the effect of helium flashes, especially since the core mass is so nearly that of the entire star. It is tempting to explain the abrupt appearance of strong $\mathrm{He} \mathrm{I}$ and $\mathrm{He} \mathrm{II}$ lines in the sdO stars as arising from deep mixing which breaks down the otherwise stable atmospheric stratification.

It would be impossible to thank adequately the numerous associates for whose advice and suggestions we wish to express our deep gratitude. Without the enormous advances in stellar atmosphere computations, this quantitative classification method would not have been possible; we are grateful to those who developed the ATLAS program, to Dimitri Mihalas for frequent advice, to Deane Peterson and Harry Shipman for specially computed models, and to Daniel Klinglesmith for his unpublished models. We are obviously indebted to many who helped refine the temperature scale, or worked on similar problems. We must also express our gratitude to Marilynne Rice for her skill and patience in preparing this manuscript. And finally, we are grateful to the memory of Henry Norris Russell, who always insisted on the primacy of quantitative observations in astrophysics.

J. L. G. wishes to express his gratitude for the leisure and instruction provided by his visit to NORDITA (Copenhagen) in 1972, and to the Associates of the California Institute of Technology for their support of the Lee A. DuBridge Professorship. A. I. S. is grateful for the opportunity to work at IOTA (Cambridge) during the summers of 1969 and 1970.

\section{APPENDIX}

\section{RADIAL VELOCITIES AND KINEMATICS}

The spectroscopic data on individual stars and groups of stars are given in tables A1-A6.

Our radial velocities used in the previous kinematical discussions are of variable quality. The original intention of the search for FB stars certainly was not to provide velocities, and no velocity standards were taken. The coudé spectra of the brighter stars should be quite reliable, however, except when few or poor lines are measurable. The sdB and sdO stars have shallow, weak lines. Usually only one spectrum was available for each star. Comparisons are difficult. Some measures of our early plates were made by Berger (1963); low-dispersion velocities are found in Slettebak, Bahner and Stock (1961), and cooler HBA velocities are published by Philip (1969). Consequently, we decided to present a large number of velocities, not only the coudé, but some of relatively low quality, based on prime-focus spectra of dispersions 90 (N1) and 190 (N2) $\AA \mathrm{mm}^{-1}$.

Where measures of a star from more than one coudé plate were available, the mean difference from the mean was found to be $\pm 2.4 \mathrm{~km} \mathrm{~s}^{-1}$ for the Pd, $\pm 1.2 \mathrm{~km} \mathrm{~s}^{-1}$ for the Pc spectra. These were usually for brighter stars, spectroscopically normal, with many lines visible. It is probable that some stars have variable velocities, at coudé scale, notably FB $46\left(+22-+44 \mathrm{~km} \mathrm{~s}^{-1}\right)$, an apparently He-rich star FB 153, and FB 158 (-34--61 $\mathrm{km} \mathrm{s}^{-1}$ ) a globular-cluster giant. Of those studied on N2 or N1 spectra, FB 89 is a known spectroscopic binary, while FB 151, FB 157, and FB 160 may also be velocity variables.

For statistical purposes it is necessary to estimate the velocity errors of the fainter stars, measured at 90 or $190 \AA \mathrm{mm}^{-1}$. Measuring spectra, in direct $(D)$ and reverse $(R)$ directions, on the Grant comparator, gives a systematic difference $D-R$. The dispersion in $\left(D_{i}-\right.$ $\left.R_{i}\right)-\langle D-R\rangle$ is found to be $\pm 12 \mathrm{~km} \mathrm{~s}^{-1}$. A study of the deviation of individual lines from the mean velocity showed that a single measure in one direction had a mean error ranging from \pm 25 (for $\mathrm{H} \delta$ ) to $\pm 45 \mathrm{~km} \mathrm{~s}^{-1}$ (for $\lambda \lambda 4471,4686$ ). Each line was measured at least four times (direct and reverse), so that a $\pm 22 \mathrm{~km} \mathrm{~s}^{-1}$ error per line measured is expected. The number of lines seen on prime-focus spectra could be as low as 5, and averages 9; therefore we expect about $\pm 10 \mathrm{~km} \mathrm{~s}^{-1}$ from internal errors alone. The formal mean error per plate, in fact, averaged $\pm 9 \mathrm{~km} \mathrm{~s}^{-1}$. The external errors will be larger. Measures of FB 89, at the prime focus, mostly at $190 \AA \mathrm{mm}^{-1}$, provide the largest single group of homogeneous residuals (Greenstein 1973); many of these plates were measured by Mrs. Knox. If we assume the velocity curve to be a precisely repetitive sine curve, with a semi-amplitude of $130 \mathrm{~km} \mathrm{~s}^{-1}$, the residuals can be evaluated for each epoch. The $\mathrm{Pe}\left(38 \AA \mathrm{mm}^{-1}\right)$ spectra showed a dispersion of $\pm 22 \mathrm{~km} \mathrm{~s}^{-1}$ in residuals about the sinusoidal velocity curve; the $\mathrm{N} 1\left(90 \AA \mathrm{mm}^{-1}\right)$ have residuals with dispersion of $\pm 24 \mathrm{~km} \mathrm{~s}^{-1}$, and the N2 $\left(190 \AA \mathrm{mm}^{-1}\right) \pm 26 \mathrm{~km} \mathrm{~s}^{-1}$. The reason is that the number of lines is limited usually to six or seven and they are seen as somewhat rotationally broadened on the $\mathrm{Pe}$ spectra. The external velocity error, for a star like FB 89 (an apparently normal B star) including true deviations from a sinusoidal velocity curve is about $\pm 25 \mathrm{~km} \mathrm{~s}^{-1}$ for either the N1 or N2 series. Note that the external errors are about 2.5 times the internal errors estimated above, a not unreasonable ratio, given both the nature of the spectra and of the prime-focus nebular spectrograph. This is a solid-Schmidt, field-flattened spectrograph, with four internal reflections. It may be subject 


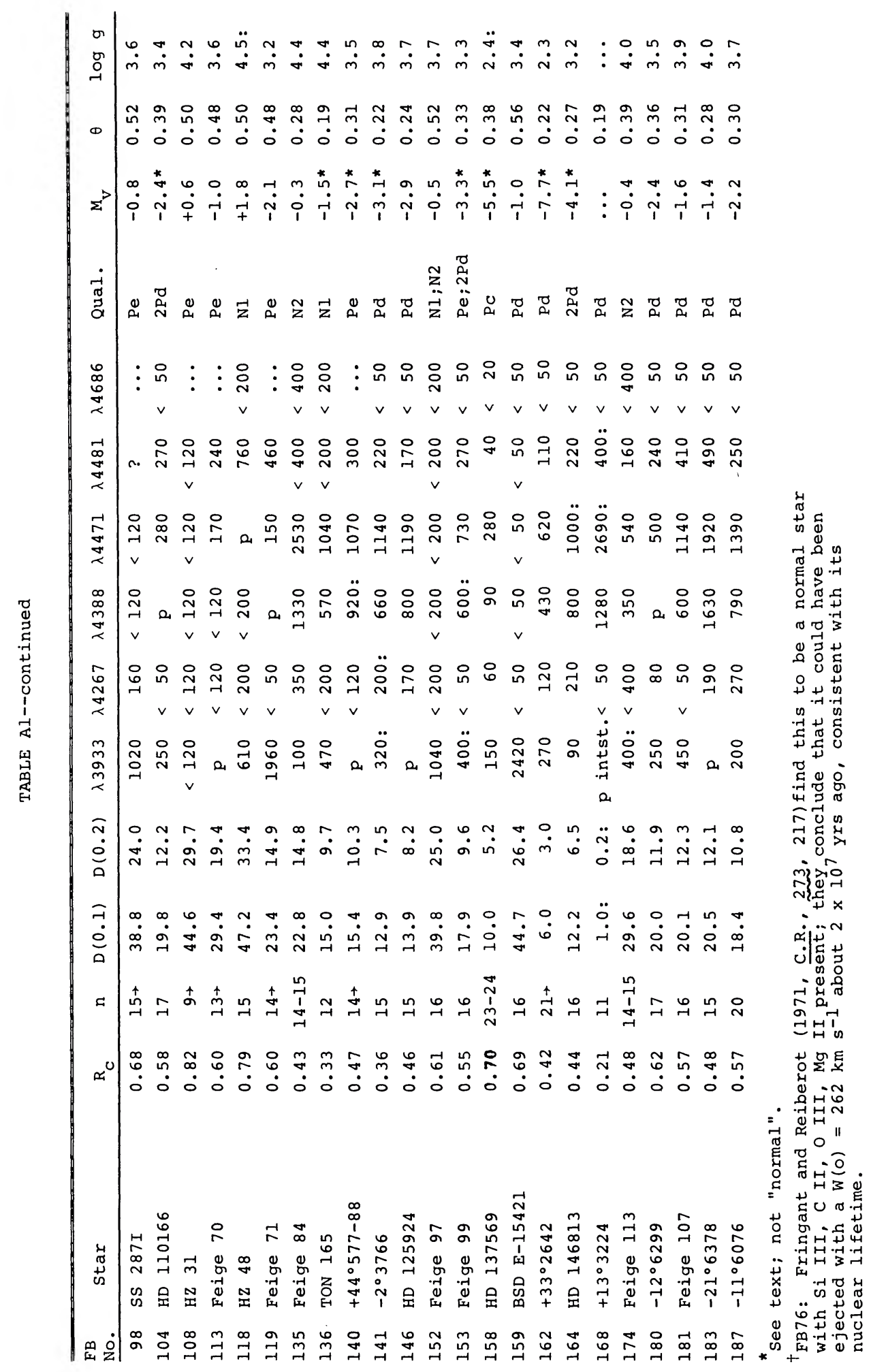

(C) American Astronomical Society - Provided by the NASA Astrophysics Data System 


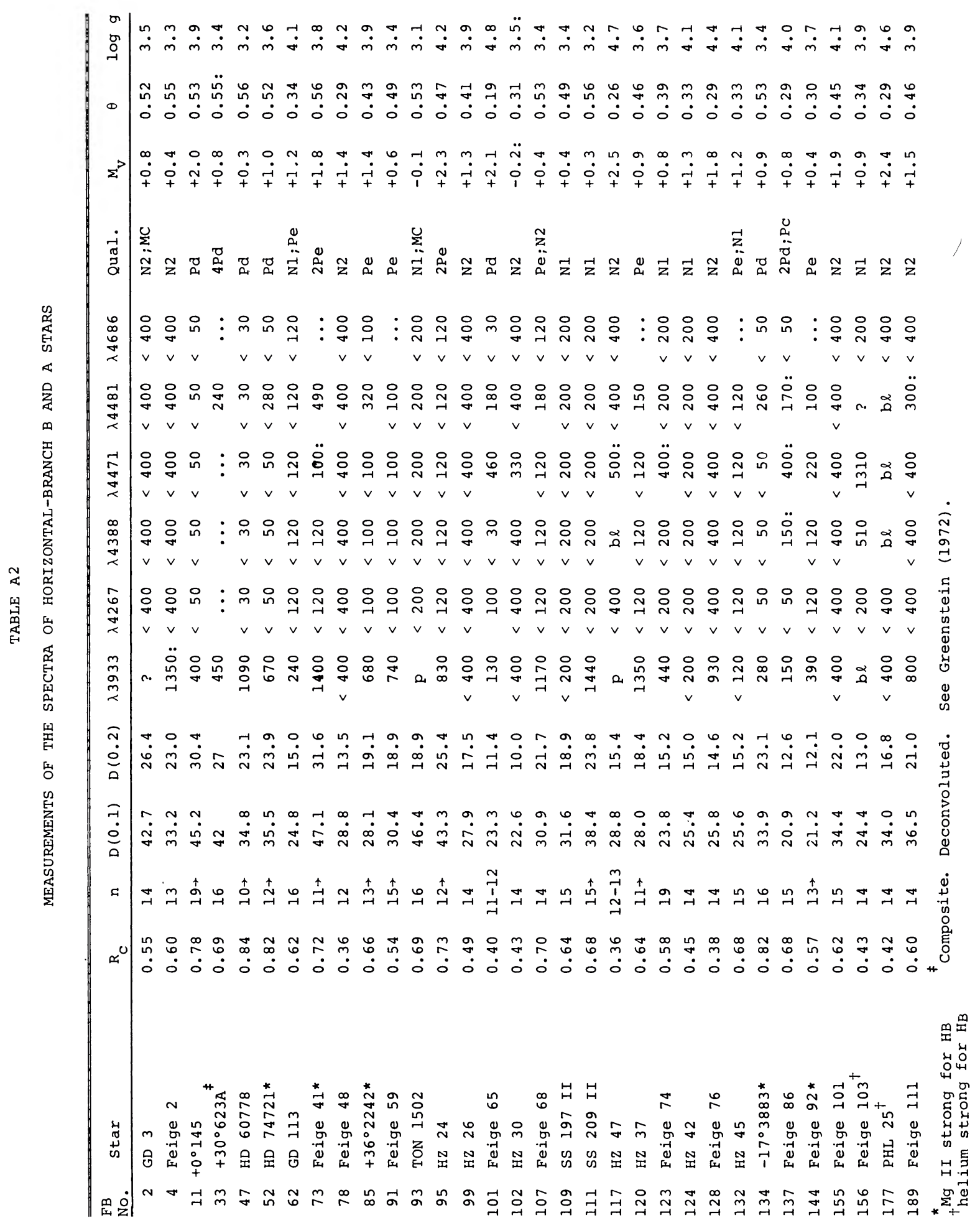




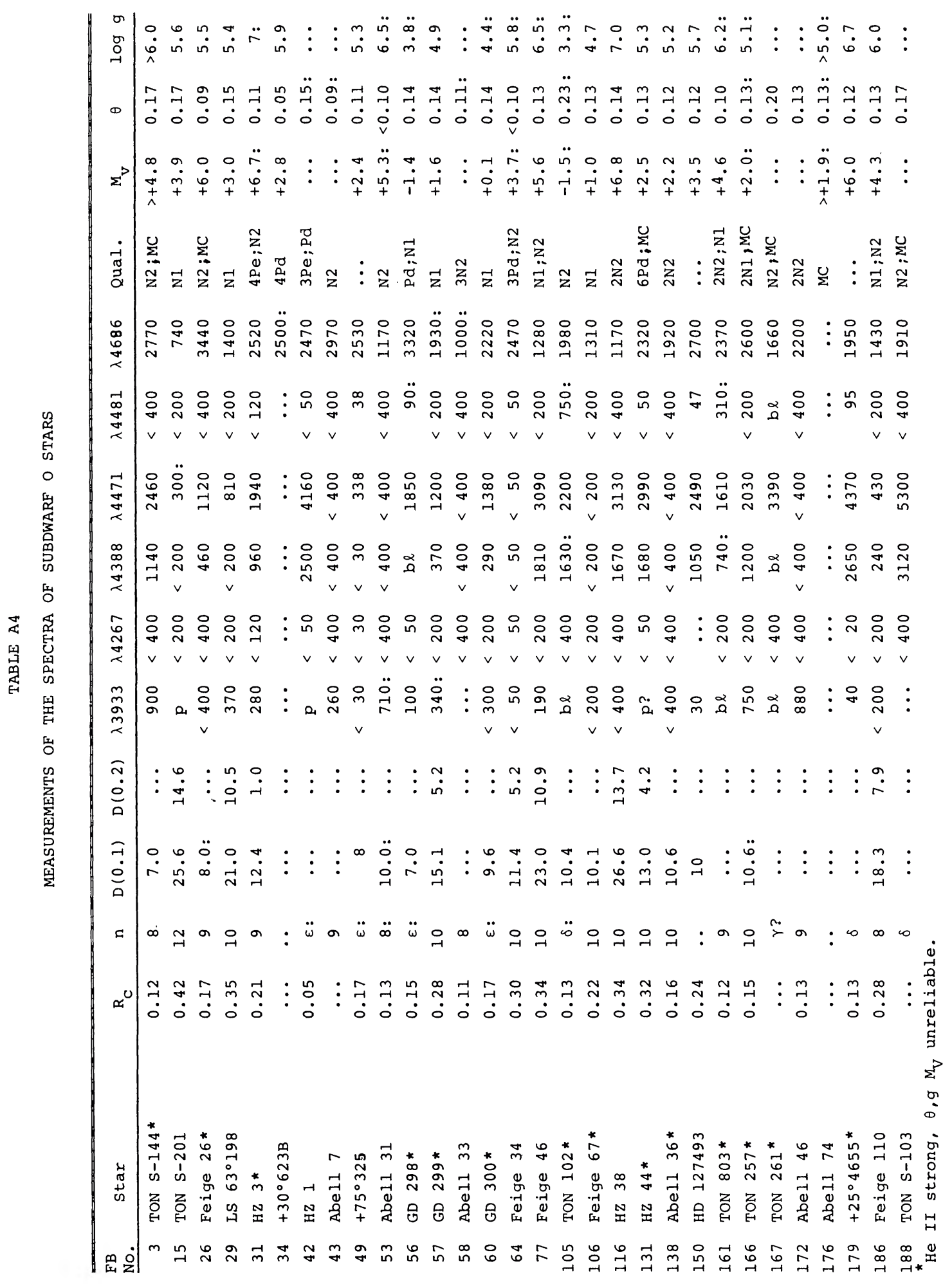

202 


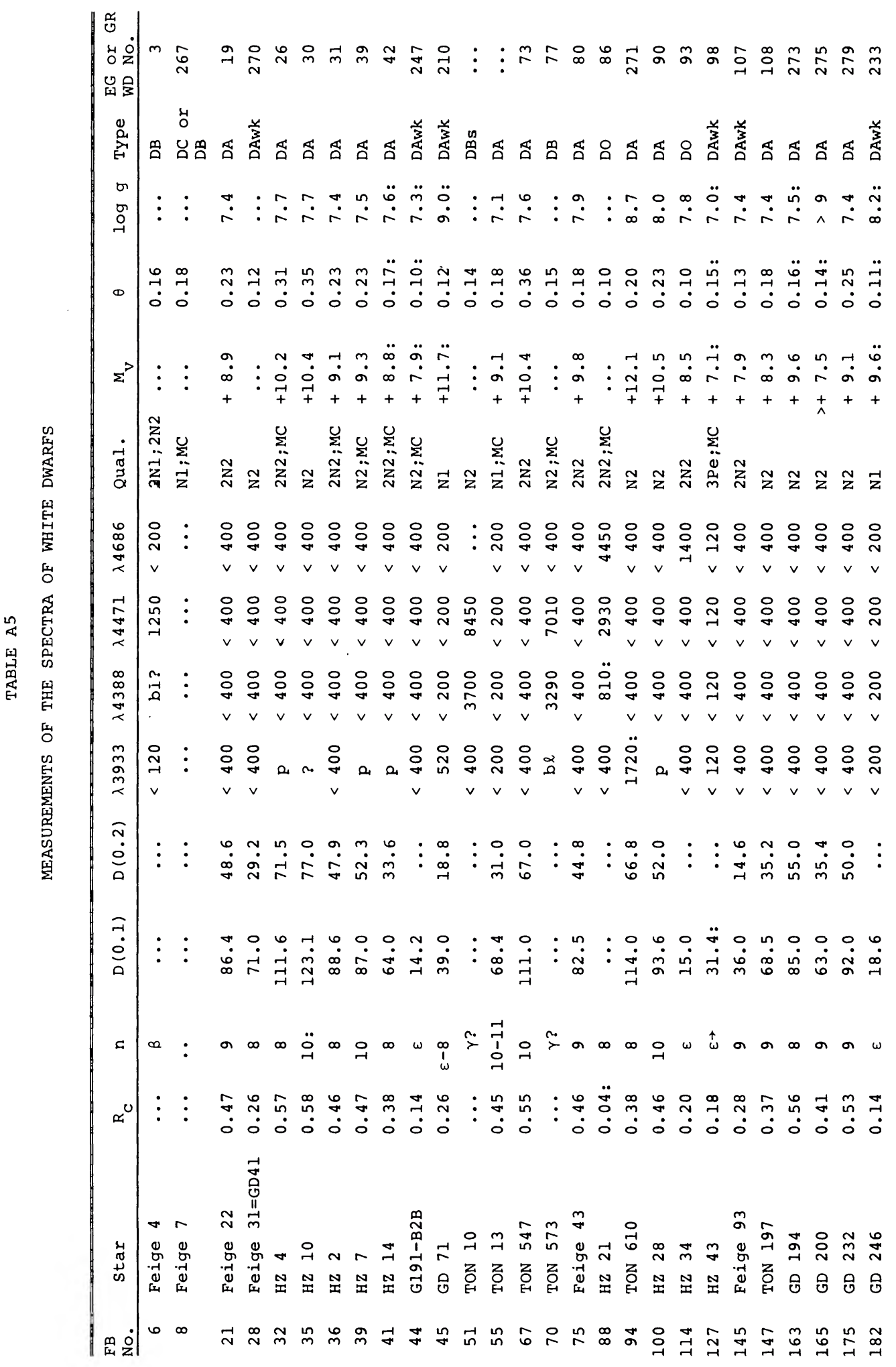




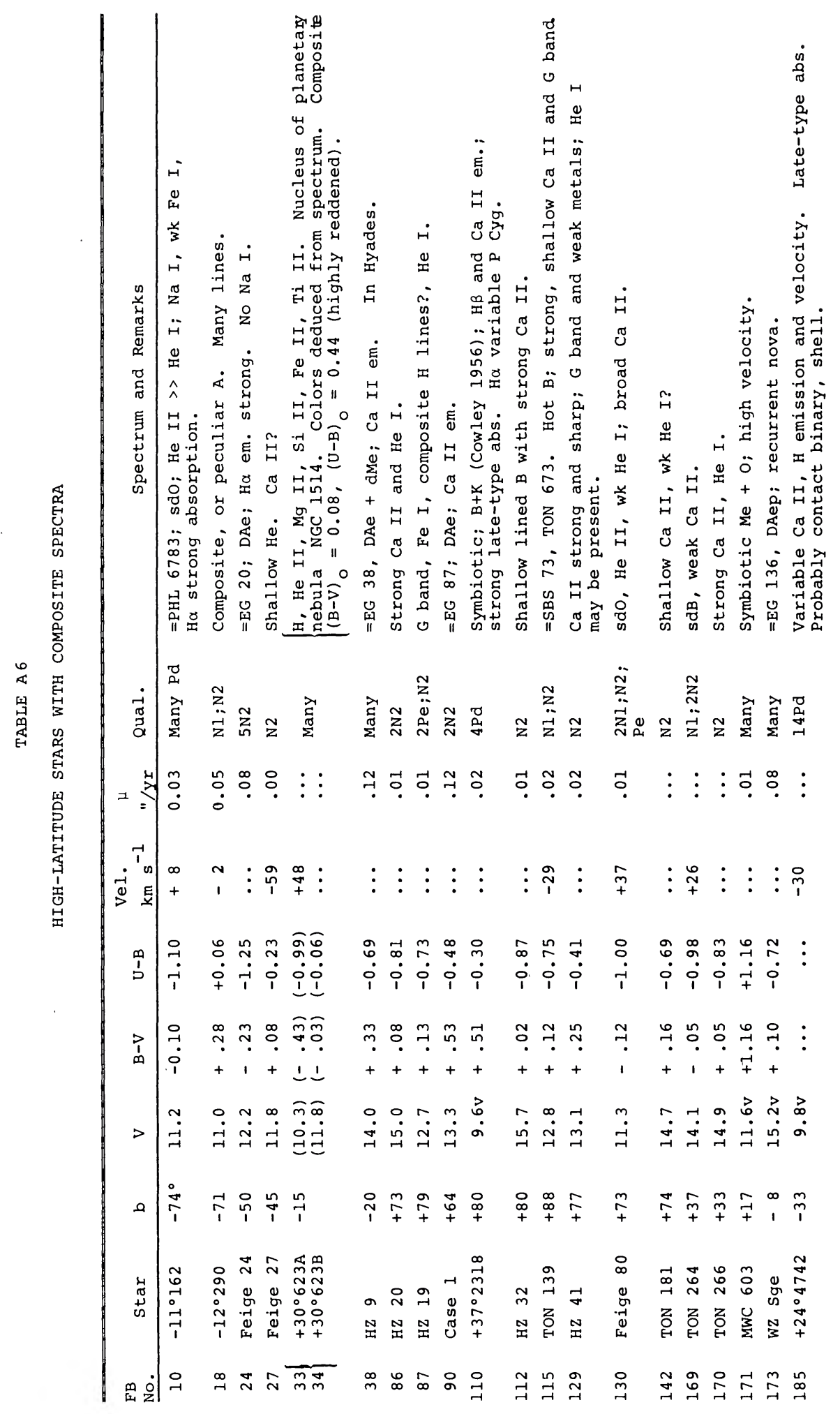

204 
to mechanical instabilities, but probably not huge ones. The comparison lines are put on at the beginning and end of the stellar exposure.

All measures were made by Grace Vess Knox, to whom we wish to express our deep gratitude. Plates were measured on a Grant comparator with a rotation by $180^{\circ}$ giving the two directions. The comparison source at the coude was a Pfund iron arc and at the prime focus a hydrogen-helium tube. Reduction, by computer, involved fitting a 4th- or 5th-order polynomial, necessary since the fields of both the Pe and $\mathrm{N}$ series have severe distortion. Trimble and Greenstein (1972), measuring DA dwarfs, found a systematic run of shifts with quantum number. Mrs. Knox found earlier that the displacements of $\mathrm{H} \beta$ from the mean velocity on N2 spectra of horizontal-branch A stars amounted to $+40 \mathrm{~km} \mathrm{~s}^{-1}$; present data for HBB and sdB stars give $+38 \mathrm{~km} \mathrm{~s}^{-1}$. For $\mathrm{H} \epsilon, \mathrm{H} \zeta, \mathrm{H} \eta$ the HBA's gave $-0.2 \mathrm{~km} \mathrm{~s}^{-1}$, and the present measures of hotter stars (shallower lines) give $-6.4 \pm 7.1 \mathrm{~km} \mathrm{~s}^{-1}$, in good agreement. We have therefore omitted $\mathrm{H} \beta$ from the means for $\mathrm{N} 1$ and $\mathrm{N} 2$ velocities given in table $\mathrm{A} 7$, and weighted $\mathrm{H} \zeta$ and $\mathrm{H} \eta$ at one-half.

Systematic differences between our results (at all dispersions) and those of Philip (1969) for four HBA stars are GS $-\mathrm{P}=-18 \pm 11 \mathrm{~km} \mathrm{~s}^{-1}$; his measure of SS 202 II may be erroneous, differing from our $90 \AA$ $\mathrm{mm}^{-1}$ plate by $+154 \mathrm{~km} \mathrm{~s}^{-1}$. For six stars in common with other published lists, including Slettebak, Bahner, and Stock (1961), the difference GS - other $=-17 \pm$ $6 \mathrm{~km} \mathrm{~s}^{-1}$; the SBS 73 published measurement $(-250$ $\mathrm{km} \mathrm{s}^{-1}$ ) may be erroneous.

In addition, we measured and give at the end of table A7 velocities of 18 stars not members of the FB lists. Some are HBA stars too cool for our methods of analysis; the HBB stars lacked colors. Only visual classifications of this group of spectra were made; eight of the 18 have also been classified as HBA by Philip (1969). Some of the HBA stars are from Kodaira, Greenstein, and Oke (1969) with new measurements of velocity. These relatively bright stars, some selected for large motions, seem to be kinematically different from the majority of our hotter HBA and HBB stars of the FB list. However, the dispersion in radial velocity for the non-FB cool HBA stars is $\pm 126 \mathrm{~km} \mathrm{~s}^{-1}$ in agreement with Philip's (1969) value of $\pm 113 \mathrm{~km} \mathrm{~s}^{-1}$; these are clearly selected for high velocity.

The dispersions, $\sigma$, in radial velocity, and the group means are given in table A8; they also are corrected for an average measuring error of $\pm 25 \mathrm{~km} \mathrm{~s}^{-1}$ and given as $\sigma^{\prime}$. Discussions of the kinematics of individual types of stars will be found in the relevant sections and some general considerations below. The differences of $\langle\rho\rangle$ from zero are marginally significant. It is not impossible that the wavelengths of $\mathrm{He} I$ lines are affected by pressure shifts in sdO stars; laboratory wavelengths were used throughout. Normal stars at high galactic latitude have large velocity dispersions, and if the runaway-star phenomenon is involved, the solar motion may be quite large. The asymmetric distribution of our stars between north and south galactic latitudes and their concentra- tion towards the galactic polar caps should also be remembered. The sdO and sdB stars are apparently and intrinsically fainter than our normal stars, and on the average closer. They should exhibit the kinematic properties of the halo. In this table, unlike table 5 in the text, we did not resolve the radial velocities for the $W$-components, but consider their dispersion as near $\sigma_{W}$, since the mean galactic latitude is so high. The total $\sigma$ for the extended-horizontal-branch, i.e., the HBA, $\mathrm{HBB}$, sdB, and sdO stars is $60 \mathrm{~km} \mathrm{~s}^{-1}$, which is less than that of the normal stars of table A1. Since many of the latter are bright objects measured on coudé spectra, it is clear that the normal stars are, in fact, a strange group, as discussed in § VIII. Whether the difference in $\sigma^{\prime}$ between $\mathrm{sdB}$ and the $\mathrm{HB}$ and $\mathrm{sdO}$ stars is significant is difficult to establish, given the small size of the samples.

To convert proper motions into estimated luminosities we need the transverse velocities. In a single Gaussian of dispersion $\sigma(\rho)$ in radial velocity, there are simple relations giving the total and tangential motions from the radial velocities (e.g., Greenstein 1956). These are very poor for our sample, since the mean galactic latitude $\left(66^{\circ}\right.$ for $\mathrm{HB}, \mathrm{sdB}, 53^{\circ}$ for $\mathrm{sdO}$ stars $)$ is so high that the tangential motion is dominated by the proper motions. In earlier sections ( $\$ \S \mathrm{IX}$ and $\mathrm{X}$ for $\mathrm{HB}$, sdB, sdO stars) we use a ratio $\left\langle V_{T}\right\rangle / \sigma(\rho)$ which we evaluate from those stars for which the proper motion is suffciently accurate to determine $U, V, W$. The standard ellipsoidal distribution (Smart 1968), neglecting the deviation of the vertex, is such that if the mobility in the $U$-direction is taken as $\sigma_{U}=1, \sigma_{V}=0.65$, and $\sigma_{W}=0.50$. Then $\sigma_{W} /\left(\sigma_{U}{ }^{2}+\sigma_{V}\right)^{1 / 2}=0.42$. We have an approximation to $\sigma_{W}$, from $\sigma_{\rho}$, and therefore to $\left\langle V_{T}{ }^{2}\right\rangle^{1 / 2}$. In the use of reduced proper motions we have as basic data the motions (reduced to absolute) from the invaluable table by Luyten (1969). Using his estimates of the errors in his motions, we were able to choose those with errors $\leq 0$ ". 010 , and to compute from our spectroscopic $M_{V}$ the $U, V, W$ components. The results can be compared with expectations by evaluating either $\sigma_{W} /\left\langle\left(U^{2}+V^{2}\right)\right\rangle^{1 / 2}$ or $\sigma(|W|) /\left\langle\left(U^{2}+V^{2}\right)^{1 / 2}\right\rangle$. In radial velocity, the ratio between mean speeds and rms speeds in a single-drift Gaussian is 0.87 . We find that this ratio $\sigma(|W|) / \sigma(W)$ is, in fact, nearly as expected. In tangential motions, the ratio is 0.96 . Because of the asymmetrical distribution of $V$, which must be largely negative for high velocity stars, the most directly useful quantity derived from our $U, V, W$ is $\sigma(|W|) /\left\langle\left(U^{2}+V^{2}\right)^{1 / 2}\right\rangle$. The values of the ratio $\sigma(|W|) /\left\langle\left(U^{2}+V^{2}\right)^{1 / 2}\right\rangle$ for 16 HBB stars was 0.45 , for 11 sdB stars, 0.40 . From the ellipsoidal distribution we expect the ratio to be $0.87 \times 0.42=0.37$. That is, if anything, our observed $U$ and $V$, which dominate $V_{T}$, the transverse motion, are slightly larger than expected, compared to the dispersion in radial speeds. However, in a single spheroidal Gaussian, this ratio would be 0.61 . Thus, although the argument is somewhat circular, we may conclude that these halo stars have about the same ellipsoidal velocity characteristics (i.e., flattened in the $W$-direction as compared with the $U$-direction) as the 
TABLE A7

RADIAL VELOCITIES

\begin{tabular}{|c|c|c|c|c|c|c|c|c|}
\hline FB & $\begin{array}{r}\mathrm{Vel}^{-1}-1 \\
\mathrm{~km} \mathrm{~s}\end{array}$ & Qual. & Type \& Remarks & FB & $\begin{array}{r}\mathrm{Vel} \\
\mathrm{km} \mathrm{s}=1\end{array}$ & Qual. & Type \& 1 & Remarks \\
\hline 1 & +24 & $\mathrm{~N} 2$ & $s d B$ & 69 & -48 & $\mathrm{~N} 2$ & $\mathbf{s d B}$ & . \\
\hline 2 & -61 & N2 & HB & 72 & +130 & N2 & $\mathrm{N}$ & \\
\hline 5 & -6 & $\mathrm{Pd}$ & $\mathrm{N}$ & 73 & -25 & $2 \mathrm{Pe}$ & HB & \\
\hline 7 & +91 & $2 \mathrm{Pd}$ & $\mathrm{N}$ & 74 & +22 & $\mathrm{~N} 1$; 2 & $\mathrm{~N}$ & \\
\hline 9 & -8 & $3 \mathrm{Pd} ; \mathrm{Pc}$ & sdB;BSS & 76 & +248 & $\mathrm{Pe}$ & N;I.S. & $-42:$ \\
\hline 10 & +8 & $\mathrm{Pd} ; 2 \mathrm{Pe}$ & Co & 77 & +89 & $\mathrm{~N} 1 ; \mathrm{N} 2$ & sdo & \\
\hline 11 & -259 & $\mathrm{Pd}$ & $\mathrm{HB}$ & 78 & -27 & $\mathrm{~N} 2$ & $\mathrm{HB}$ & \\
\hline 12 & -83 & $\mathrm{NI}$ & $\mathrm{sdB}$ & 79 & +58 & $\mathrm{Pe}$ & N;I.S. & -7 \\
\hline 14 & +26 & $\mathrm{~N} 1$ & $\mathrm{sdB}$ & 80 & +24 & $\mathrm{Pd} ; \mathrm{Pe}$ & $\mathrm{N}$ & \\
\hline 15 & -106 & $\mathrm{~N} 1$ & sdo & 82 & -66 & $\mathrm{~N} 2$ & $s d B$ & \\
\hline 16 & -7 & $\mathrm{Pd}$ & $\mathrm{N}$ & 83 & +34 & $\mathrm{Pd}$ & $\mathrm{N}$;I.S. & $-2:$ \\
\hline 17 & +2 & $\mathrm{Pd} ; \mathrm{NI}$ & $\mathrm{N}$ & 84 & -18 & N2 & $\mathrm{sdB}$ & \\
\hline 18 & -2 & $\mathrm{~N} 1 ; \mathrm{N} 2$ & Co & 85 & -3 & $\mathrm{Pe}$ & $\mathrm{HB}$ & \\
\hline 19 & +101 & $\mathrm{Pe}$ & $\mathbf{s d B}$ & 87 & -19 & $\mathrm{Pe}$ & Co & \\
\hline 20 & +8 & $\mathrm{~N} 1 ; \mathrm{N} 2$ & $\mathbf{s d B}$ & 89 & -1 & $10 \mathrm{~N} 2$ & $\mathrm{~N}$-spect & $\therefore$ Binary \\
\hline 22 & +6 & $\mathrm{Pd} ; \mathrm{N} 2$ & $\mathrm{~N}$ & 91 & +22 & $\mathrm{Pe}$ & $\mathrm{HB}$; I.S. & -24 \\
\hline 23 & -12 & $\mathrm{Pe}$ & $\mathrm{N}$ & 92 & +16 & $\mathrm{Pd}$ & $\mathrm{N}$ & \\
\hline 25 & $-\quad 5:$ & $\mathrm{Pe}$ & $\mathrm{N}$ & 93 & -70 & $\mathrm{Nl}$ & $\mathrm{HB}$ & \\
\hline 27 & -59 & N2 & Co & 95 & -40 & $\mathrm{Pe}$ & HB & \\
\hline 29 & -71 & $2 \mathrm{~N} 1$ & sdo & 96 & +91 & $2 \mathrm{Pd}$ & $\mathrm{N}$ & \\
\hline 30 & +22 & $2 \mathrm{Pd}$ & $\mathrm{N}$ & 97 & +42 & $2 \mathrm{Pd} ; \mathrm{Pe}$ & $\mathrm{N} ; \mathrm{I} . \mathrm{S}$ & -8 \\
\hline 31 & -120 & $4 \mathrm{Pe} ; \mathrm{N} 2$ & sdo & 98 & -137 & $\mathrm{Pe}$ & $\mathrm{N}$ & \\
\hline $\begin{array}{l}33 \\
34\end{array}$ & $\begin{array}{l}+48 \\
+\quad 48\end{array}$ & $3 \mathrm{Pd}$ & $\mathrm{HB}+\mathrm{sdO}, \mathrm{Co}$ & 99 & +72 & $\mathrm{~N} 2$ & $\mathrm{HB}$ & \\
\hline 37 & +38 & $3 \mathrm{Pe}$ & N;I.S. +16 & 101 & +55 & $\mathrm{Pd}$ & $\mathrm{HB}$ & \\
\hline 40 & -18 & $3 \mathrm{Pe}$ & N;I.S. -7 & 102 & -111 & $\mathrm{~N} 2$ & $\mathrm{HB}$ & \\
\hline 42 & -10 & $3 \mathrm{Pe}$ & sdo & 103 & -11 & $2 \mathrm{PC} ; \mathrm{Pd}$ & $\mathrm{sdB}$ & \\
\hline 46 & $\begin{array}{l}+44, \\
+\quad 27\end{array}$ & $\mathrm{Pd} ; \mathrm{Pe}$ & $\begin{array}{l}\text { NiI.S. +26; } \\
\text { var.? }\end{array}$ & $\begin{array}{l}104 \\
105\end{array}$ & $\begin{array}{l}-86 \\
-72\end{array}$ & $\begin{array}{l}2 \mathrm{Pd} \\
\mathrm{N} 2\end{array}$ & $\begin{array}{l}\text { N } \\
\text { sdo }\end{array}$ & \\
\hline 47 & +43 & $\mathrm{Pd}$ & HB & 106 & +22 & $\mathrm{~N} 1$ & sdo & \\
\hline 48 & -49 & N2 & $\mathrm{sdB}$ & 107 & +88 & $\mathrm{Pe} ; \mathrm{N} 2$ & $\mathrm{HB}$ & \\
\hline 49 & $-44:$ & $\mathrm{Pd}$ & sdo; VP & 108 & $+26::$ & $\mathrm{Pe}$ & $\mathrm{N}$ & \\
\hline 50 & -22 & $\mathrm{~N} 1$ & $\mathrm{~N}$ & 109 & +60 & $\mathrm{~N} 1$ & HB & \\
\hline 52 & +36 & $\mathrm{Pd}$ & $\mathrm{HB}$ & 111 & -75 & $\mathrm{~N} 1$ & $\mathrm{HB}$ & \\
\hline 54 & -39 & $\mathrm{Pd}$ & $\mathrm{N}$ & 113 & -8 & $\mathrm{Pe}$ & $\mathrm{N}$ & \\
\hline 56 & -43 & $2 \mathrm{Pd} ; \mathrm{NI}$ & sdo & 115 & -29 & $\mathrm{~N} 1 ; \mathrm{N} 2$ & Co & \\
\hline 57 & +11 & N2 & sdo & 116 & +64 & $2 \mathrm{~N} 2$ & sdo & \\
\hline 60 & $+17:$ & NI & sdo & 117 & -62 & $\mathrm{~N} 2$ & $\mathrm{HB}$ & \\
\hline 61 & +62 & $\mathrm{Pe}$ & $\mathbf{s d B}$ & 118 & -53 & $\mathrm{~N} 1$ & $\mathrm{~N}$ & \\
\hline 62 & -13 & $\mathrm{Nl} ; \mathrm{Pe}$ & $\mathrm{HB}$ & 119 & -12 & $\mathrm{Pe}$ & $\mathrm{N}$ & \\
\hline 63 & +49 & $\mathrm{Pd}$ & $\mathrm{N} ; \mathrm{I} . \mathrm{S} .-13$ & 121 & -33 & $\mathrm{~N} 2$ & $\mathbf{s d B}$ & \\
\hline 64 & +1 & $3 P d ; N 2$ & sdo & 123 & -83 & $\mathrm{Nl}$ & $\mathrm{HB}$ & \\
\hline 65 & +38 & $\mathrm{Pd} ; \mathrm{Pe}$ & $\mathrm{N}$ & 124 & -88 & $\mathrm{Nl}$ & HB & \\
\hline 66 & +79 & $\mathrm{Pe}$ & $\mathrm{N}$ & 125 & -111 & N2 & $\mathrm{sdB}$ & \\
\hline 68 & +208 & N2 & $\mathrm{N}$ & 128 & +39 & $\mathrm{~N} 2$ & HB & \\
\hline
\end{tabular}


TABLE A7-continued

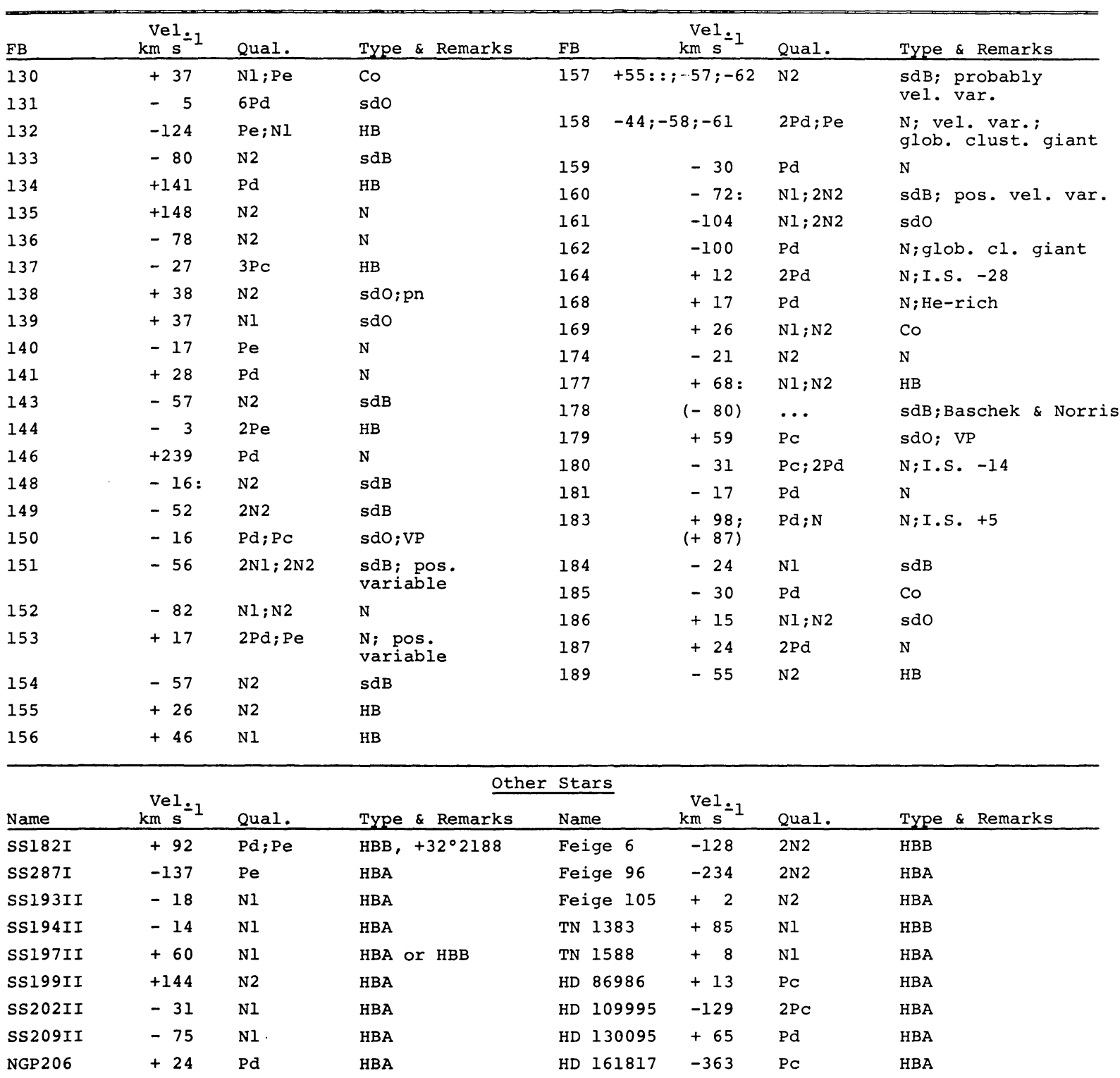

TABLE A8

Means and Dispersions in Radial Velocities for Analysis of Reduced Proper Motions $\left(\mathrm{km} \mathrm{s}^{-1}\right)$

\begin{tabular}{ccccrrr}
\hline \hline Group & $\langle\rho\rangle$ & m.e. & \multicolumn{1}{c}{$\sigma$} & \multicolumn{1}{c}{$\sigma^{\prime}$} & $n$ \\
\hline FB, HBA, HBB $\ldots \ldots \ldots$ & -14.9 & \pm 14.0 & \pm 78 & \pm 74 & 31 \\
non-FB, HBA, HBB $\ldots \ldots$ & -35.5 & \pm 29.6 & 126 & 124 & 18 \\
FB, sdB $\ldots \ldots \ldots \ldots \ldots \ldots$ & -30.1 & \pm 4.9 & 49 & 42 & 24 \\
FB, sdO $\ldots \ldots \ldots \ldots \ldots \ldots$ & -8.8 & \pm 13.1 & 60 & 55 & 21 \\
FB, all EHB $\ldots \ldots \ldots \ldots$ & -18.3 & \pm 7.5 & 65 & 60 & 76 \\
FB, normal........... & +27.3 & \pm 12.2 & 79 & 75 & 42
\end{tabular}

body of normal stars, and that the absolute magnitudes assumed are approximately correct. For example, an error in distance by a factor of 0.7 changes both $U$ and $V$ by about 30 percent, beyond the range of acceptability, given our $U, V, W$ values in earlier sections, and the ratios to $\sigma(|W|)$ in table A8. In the conventional formula for the use of reduced proper motions, defining $H$ as

$$
H_{V}=m_{V}+5 \log \mu,
$$

the mean $M_{V}$ is obtained from

$$
\left\langle M_{V}\right\rangle=\left\langle m_{V}+5 \log \mu\right\rangle+8.39-5\left\langle\log V_{T}\right\rangle .
$$


The difference $\left\langle\log V_{T}\right\rangle-\log \left\langle V_{T}\right\rangle=-0.06$ from a small sample, but we will not make this correction. Using the theoretical ratio of $\sigma(W) /\left\langle\left(U^{2}+V^{2}\right)^{1 / 2}\right\rangle$, $\left\langle V_{T}\right\rangle$ can be taken as $(0.42 \times 0.96)^{-1}$ i.e., $\left\langle V_{T}\right\rangle=2.48$ $\sigma^{\prime}(\rho)$ from table A8. This gives a possible direct answer but we might worry about the projection factor between $\rho$ and $W$; at the mean galactic latitude of $66^{\circ}$ another 9 percent correction is required in $\sigma(W) / \sigma(\rho)$ giving $\left\langle V_{T}\right\rangle=2.70 \sigma^{\prime}(\rho)$. However, the larger mobility in $U$ begins to appear in the radial component, in certain directions, at $b=66^{\circ}$. The factor 2.70 may be too large and 2.48 too small; $0.5 \pi$ from the spherical velocity distribution is clearly too small. The range in the constant in equation (A3) is from 6.2 to 6.6, probably. For a group with larger spread in galactic latitude, however, even 7.0 would be plausible. An approximate formula is

$$
\left\langle M_{V}\right\rangle=\left\langle m_{V}+5 \log \mu\right\rangle+6.23-5 \log \sigma^{\prime}(\rho) .
$$

The actual $U, V, W$ are more directly useful, and are given in earlier sections; they have been used in some cases to correct $M_{V}$, if the motions derived are too large for an acceptable galactic model. In general the observed ratios of radial to transverse motion just mentioned and used were derived after reducing the distance to a few sdB stars, and especially sdO stars, for which the total motions clearly exceeded $300 \mathrm{~km} \mathrm{~s}^{-1}$. There is some statistical loading in this process; but it should be also remembered that spuriously large transverse motions will appear at great distances just from the propermotion errors. At a distance of $d$ in $\mathrm{kpc}$, an error of 0 . 010 in $\mu$ produces a spurious tangential motion of $47 d \mathrm{~km} \mathrm{~s}^{-1}$ at the galactic pole. A star of $M_{V}=+2$ at $m_{V}=14$ is at $d=2.5$, and has spurious $V_{T} \simeq 117$ $\mathrm{km} \mathrm{s}^{-1}$.

We have some impression that the sdO stars are a less homogeneous group, kinematically, than the other EHB stars; only seven can have $U, V, W$ determined and some required adjustments in $M_{V}$. Some apparently bright sdO stars have small proper motions. We hope to investigate the kinematics of all these groups in greater detail.

\section{REFERENCES}

Abell, G. 1966, $A$ p. J., 144, 259.

Auer, L. H., and Mihalas, D. 1973, A p.J.Suppl., 25, 433, No. 223.

Baschek, B., and Norris, J. 1970, Ap. J. Suppl., 19, 327, No. $176(\mathrm{BN})$.

Baschek, B., Sargent, W. L. W., and Searle, L. 1972, Ap. J., 173, 611 (BSS).

Baschek, B., and Searle, L. 1969, Ap.J., 155, 537.

Berger, J. 1963, Pub. A.S.P., 75, 393.

Biermann, P., and Kippenhahn, R. 1971, Astr. and Ap., 14, 32.

Blaauw, A. 1961, B.A.N., 15, 265.

Blanco, V. M., Demers, S., Douglass, G. G., and Fitzgerald, M. P. 1968, Pub. U.S. Naval Obs., Vol. 21.

Böhm-Vitense, E. 1968, Ap.J., 150, 483.

Cannon, R. D., and Stobie, R. S. 1973, M.N.R.A.S., 162, 227.

Chavira, E. 1958, Bol. Obs. Tonantzintla y Tacubaya, No. 17.

-. 1959, ibid., No. 18.

Clayton, D. D. 1968, Principles of Stellar Evolution and Nucleosynthesis (New York: McGraw-Hill).

Cowley, C. R. 1956, Pub. A.S.P., 68, 537.

. 1958, A.J 63, 484 .

Danziger, I. J. 1970, Ann. Rev. Astr. and Ap., 8, 161.

Danziger, I. J., and Jura, M. A. 1970, $A$ p. J., 161, 997.

Demarque, P., and Mengel, J. G. 1971, Ap.J., 164, 469.

- 1972 , ibid., 171, 583.

Eggen, O. J. 1968a, Ap.J. Suppl., 16, 97, No. 143. $1968 b, A$ p.J., 153, 723 .

Eggen, O. J., and Greenstein, J. L. 1965, Ap. J., 141, 83 (EG).

. 1967, Ap.J., 150, 927.

Faulkner, J. 1966, Ap.J., 144, 978.

- 1972, ibid., 173, 401.

Feige, J. 1958, $A$ p. J., 128, 267.

Giclas, H. L., Burnham, R., Jr., and Thomas, N. G. 1965, Lowell Obs. Bull., 6, 155, and elsewhere.

Graham, J. 1970, Pub. A.S.P., 82, 1305.

-. 1972, A.J., 77, 144.

Greenstein, G. S., Truran, J. W., and Cameron, A. G. W., 1967, Nature, 213, 871.

Greenstein, J. L. 1952, Pub. A.S.P., 64, 256.

. 1956, Third Berkeley Symposium on Mathematical Statistics and Probability, ed. J. Neyman (Berkeley: University of California Press), p. 15.

C. 1960, in Stellar Atmospheres, ed. J. L. Greenstein (Chicago: University of Chicago Press), chap. 19.

. 1966, A p.J., 144, 496.

. 1968, ibid., 152, 431.
- 1969, ibid., 158, 281.

1971 , in White Dwarfs, ed. W. J. Luyten (Dordrecht:

Reidel), chap. 9.

- 1972, Ap.J., 173, 367.

Greenstein, J. L., and Eggen, O. J. 1966, in Vistas in Astronomy, Vol. 8, ed. A. Beer (Oxford: Pergamon Press), p. 63.

Greenstein, J. L., and Minkowski, R. 1964, Ap. J., 140, 1601.

Greenstein, J. L., and Münch, G. 1966, Ap.J., 146, 618.

Greenstein, J. L., Sargent, A. I., and Haug, U. 1970, Astr. and Ap., 7, 1 .

Gross, P. G. 1973, M.N.R.A.S., 164, 65.

Hanbury Brown, R., Davis, J., Allen, L. R., and Rome, J. M. 1967, M.N.R.A.S., 137, 393 .

Harman, R. J., and Seaton, M. J. 1964, Ap. J., 140, 824.

Haro, G., and Luyten, W. J. 1962, Bol. Obs. Tonantzintla y Tacubaya, No. 22.

Harris, D. L., Strand, K. Aa., and Worley, C. E. 1963, in Basic Astronomical Data, ed. K. Aa. Strand (Chicago: University of Chicago Press), chap. 15.

Heintze, J. R. W. 1969, B.A.N., 20, 154.

Herbig, G. 1969, Proc. Nat. Acad. Sci., 63, 1045.

Humason, M. L., and Zwicky, F. 1947, A P. J., 105, 85.

Hyland, A. R. 1967, thesis, Australian National University.

Iben, I., Jr., 1967a, Ann. Rev. Astr. and A p., 5, 571. $1967 b$, Science, 155, 785 .

1971, Pub. A.S.P., 83, 697.

Iriarte, B. 1959, Lowell Obs. Bull., 4, 130.

Iriarte, B., and Chavira, E. 1957, Bol. Obs. Tonantzintla y Tacubaya, No. 16.

Johnson, H. L., and Morgan, W. W. 1953, A p. J., 117, 313.

Klemola, A. R. 1962, A.J., 67, 740.

Klinglesmith, D. A. 1971, Hydrogen-Line Blanketed Model Stellar Atmospheres, NASA SP-3065 (Greenbelt, Md.: Goddard Space Flight Center) and private communication.

Kodaira, K., Greenstein, J. L., and Oke, J. B. 1969, A p. J., 155, 525.

Kowal, C. 1973, private communication.

Kurucz, R. L. 1970, Smithsonian Ap. Obs. Spec. Rept., No. 309.

L'Ecuyer, J. 1966, A p. J., 146, 845.

Lester, J. B. 1972, A p.J., 178, 743.

Luyten, W. J. 1969, A Search for Faint Blue Stars, No. 50 (University of Minnesota).

Luyten, W. J., and Anderson, J. H. 1959, Pub. Obs. Univ. Minnesota, No. 18.

Michaud, G. 1970, Ap.J., 160, 641. 
Mihalas, D. 1965, Ap.J. Suppl., 9, 321, No. 92. 1972a, NCAR Technical Note, NCAR-TN/STR-76 (Boulder: Natl. Center for Atmospheric Research). . $1972 b, A$ p.J., 176, 139.

Morgan, W. W., Keenan, P. C., and Kellman, E. 1943, An Atlas of Stellar Spectra. (Chicago: University of Chicago Press).

Morton, D. C., and Adams, T. F. 1968, Ap. J., 151, 611.

Münch, G. 1956, Ann. Rept. Dir. Mt. Wilson and Palomar Obs. (Carnegie Yrb., No. 55, p. 44).

Newell, E. B. 1969, thesis, Australian National University. 1973, Ap.J.Suppl., 26, 37, No. 228.

Norris, J. 1970, thesis, Australian National University.

Norris, J., and Baschek, B. 1970, A p.J. Suppl., 19, 305, No. 176.

O'Dell, C. R. 1968, in Planetary Nebulae, ed. D. E. Osterbrock and

C. R. O'Dell (New York: Springer-Verlag), p. 361.

O'Dell, C. R., Peimbert, M., and Kinman, T. D. 1964, Ap. J., $140,119$.

Oke, J. B. 1974, Ap.J. Suppl., No. 236, 27.

Oke, J. B., and Schild, R. E. 1970, Ap. J., 161, 1015.

Oort, J. H. 1932, B.A.N., 6, 249. . 1960, ibid., 15, 45.

Osmer, P. S., and Peterson, D. M. 1974, Ap. J., 187, 117.

Paczyński, B. 1971a, Acta Astr., 21, 1.

- 1971b, ibid., 21, 417.

Penston, M. J. 1973, M.N.R.A.S., 164, 133.

Peterson, A. V. 1969, thesis, California Institute of Technology.

Philip, A. G. D. 1968, $A p . J$. 152, 1107.

. 1969, Ap.J. (Letters), 158, L113.

Rodgers, A. W. $1971, A$ p.J., 165,581

Rood, R. T. 1973, $A$ p.J., 184, 815.

Salpeter, E. E. 1971, Ann. Rev. Astr. and Ap., 9, 127.

Sandage, A. R. 1969, A p. J., 157, 515.

. 1970, ibid., 162, 841.

Sargent, W. L. W., and Searle, L. 1967, A p. J. (Letters), 150, L33. . 1968, Ap.J., 152, 443 (SS).
1970), p. 403 .

1971, Ann. Rpt. Dir. Hale Obs., (Carnegie Yrb., Schatzman, E. 1958, White Dwarfs (Amsterdam: North Holland). Schild, R. E., Peterson, D. M., and Oke, J. B. 1971, A p. J., 166, 95 (SPO).

Searle, L., and Rodgers, A. W. 1966, Ap.J., 143, 809.

Searle, L., and Sargent, W. L. W. 1967, in The Magnetic and Related Stars, ed. R. C. Cameron (Baltimore: Mono Book Corp.), p. 219.

. 1972, Comm. Ap. and Space Phys., 4, 59.

Shipman, H. L. 1970, thesis, California Institute of Technology. 1972, A p. J., 177, 723.

Slettebak, A., Bahner, K., and Stock, J. 1961, Ap. J., 134, 195.

Smart, W. M. 1968, Stellar Kinematics (New York: Wiley).

Stoeckly, R., and Greenstein, J. L. 1968, A p. J., 154, 909.

Stothers, R. 1966, A.J., 71, 943.

Strom, S. E., and Strom, K. M. 1970, Ap. J., 159, 195.

$\longrightarrow$ 1971, Astr. and Ap., 14, 111.

Tomley, L. J. 1970, Ap. J., 162, 239.

Traving, G. 1962, Ap.J., 135, 439.

Trimble, V., and Greenstein, J. L. 1972, Ap. J., 177, 441 (TG).

Trimble, V., and Paczyński, B. 1973, Astr. and A p., 22, 9.

Vidal, C. R., Cooper, J., and Smith, E. W. 1973, A p. J. Suppl., 25,37 , No. 214.

Wegner, G. 1974, M.N.R.A.S., 166, 271

Weidemann, V. 1968, Ann. Rev. Astr. and Ap., 6, 351.

. 1971, in White Dwarfs, ed. W. J. Luyten (Dordrecht: Reidel), p. 81.

Wickramasinghe, D. T , and Strittmatter, P.A. 1972, M.N.R.A.S., 160,421 (WS).

Wright, K. O., Lee, E. K., Jacobson, T. V., and Greenstein, J. L. 1964, Pub. Dom. A p. Obs., 12, No. 7.

Zinn, R. J., Newell, E. B., and Gibson, J. B. 1972, Astr. and Ap., 18,390 .

Jesse L. Greenstein: California Institute of Technology, 1201 East California Blvd., Pasadena, CA 91109

Anneila I. Sargent: California Institute of Technology, 1201 East California Blvd., Pasadena, CA 91109 